\title{
Correlated Evolution of two Copulatory Organs via a Single Cis-Regulatory Nucleotide Change
}

Olga Nagy ${ }^{1}$, Isabelle Nuez ${ }^{1}$, Rosina Savisaar ${ }^{1 \dagger}$, Alexandre E. Peluffo ${ }^{1}$, Amir Yassin $^{2}$, Michael Lang $^{1}$, David L. Stern ${ }^{3}$, Daniel R. Matute ${ }^{4}$, Jean R. David ${ }^{2,5}$ and Virginie Courtier-Orgogozo ${ }^{1,6, *}$

One Sentence Summary: We identify one nucleotide substitution in a gene regulatory region contributing to evolutionary change of two distinct copulatory organs.

\section{Highlights:}

- We identify a gene and 3 substitutions causing genital evolution between species

- The evolved mutations lie in a pleiotropic enhancer

- One mutation decreases genital bristle number and increases leg sex comb tooth number

- This mutation disrupts a binding site for Abd-B in genitals and for another factor in legs

\section{SUMMARY}

Diverse traits often covary between species. The possibility that a single mutation could contribute to the evolution of several characters between species is rarely investigated as relatively few cases are dissected at the nucleotide level. Drosophila santomea has evolved additional sex comb sensory teeth on its legs and has lost two sensory bristles on its genitalia. We found that a single nucleotide substitution in an enhancer of the scute gene contributes to both changes. The mutation alters a binding site for the Hox protein Abdominal-B in the developing genitalia, leading to bristle loss, and for another factor in the developing leg, leading to bristle gain. Our study shows that morphological evolution between species can occur through a single nucleotide change affecting several sexually dimorphic traits.

(125 words) 
Keywords: pleiotropy, cis-regulatory, sensory organ, Drosophila, copulation, genitalia, hypandrium, sex comb, microevolution, scute, $A b d-B$

\section{RESULTS AND DISCUSSION}

"Variability is governed by many unknown laws, of which correlated growth is probably the most important."'[1] Correlated evolution of traits is widespread among taxa $[1,2]$ and can be due to pleiotropy, where a single locus causally affects several traits [3]. Pleiotropy imposes large constrains on the paths of evolution [4,5], making it crucial to assess the extent of pleiotropy to understand the evolutionary process. Empirical studies suggest that many loci influence multiple traits $[3,6,7]$ and current data cannot reject the idea that all genetic elements have pleiotropic roles $[3,8,9]$. Several pleiotropic substitutions have been associated with natural variation $[10-12,12 \mathrm{~b}]$ : most are coding changes and all underlie intraspecific changes (www.gephebase.org). Nevertheless it remains unclear whether pleiotropic mutations contribute also to interspecific evolution, as experimental evidence suggests that the mutations responsible for interspecies evolution may be less pleiotropic than the mutations underlying intraspecific variation [13].

Here we focused on male sexual bristle evolution between Drosophila yakuba and Drosophila santomea, which diverged approximately 0.5-1 million years ago [14] and can produce fertile F1 females in the laboratory [15], facilitating genetic mapping. We found that hypandrial bristles - two prominent mecanosensory bristles located on the ventral part of male genitalia in all $D$. melanogaster subgroup species - are missing in D. santomea males (Fig. 1). Examination of many inbred stocks and 10 closely related species revealed that the absence of hypandrial bristles is a derived D. santomeaspecific trait (Fig. 1, Tables S1-S2). No other genital bristle type was noticeably variable in number between $D$. yakuba and D. santomea (Fig. S1).

We performed whole-genome QTL mapping between D. santomea and D. yakuba and found that the left tip of chromosome X explains $44 \%$ of the variance in hypandrial bristle number in each backcross (confidence interval $=7 \mathrm{Mb}$ for the $D$. santomea backcross and $2.6 \mathrm{Mb}$ for the $D$. yakuba backcross, Fig. 2A). Duplication mapping in rare D. santomea-D. melanogaster hybrid males narrowed down the causal region to a $84.6 \mathrm{~kb}$ region of the achaete-scute complex $(A S-C)$ (Fig. 2B-C, Table S2).

The $A S-C$ locus contains four genes, but only two, achaete $(a c)$ and scute ( $s c$ ), are required for bristle formation [16]. Both genes are co-expressed, share cis-regulatory elements and act redundantly to specify bristles $[17,18]$. The elaborate expression pattern of $a c$ and $s c$ genes prefigures the adult bristle pattern and is controlled by numerous cis-regulatory elements [17]. We tested which of the two genes, $a c$ or $s c$, contributes to loss of bristles using null mutants in D. melanogaster. All $a c^{C A M l}$ null mutant males had 2 hypandrial bristles $(\mathrm{n}=15)$ and $s c^{M 6}$ null mutants had none $(\mathrm{n}=15)$ (Table S4), indicating that $s c$ is required for hypandrial bristle development in D. melanogaster.

We detected 64 nucleotide differences in the $s c$ coding region between D. yakuba and $D$. santomea, and all were synonymous substitutions, indicating that coding changes in $s c$ are not responsible for the evolved function of $s c$. Using molecularly mapped chromosomal aberrations, we identified a $5-\mathrm{kb}$ region located $>46 \mathrm{~kb}$ downstream of the $s c$ promoter that is required in $D$. melanogaster for hypandrial bristle development (Fig. S2, Tables S3-4). Independently we screened 55 GAL4 reporter constructs tiling the entire $A S$-C locus and identified three GAL4 lines (15E09, 054839 and $18 \mathrm{C05}$ ) that drive expression in hypandrial bristles (Fig. 2C, Tables S5-6). Only one of these lines, $18 C 05$, increased hypandrial bristle number with $U A S$-scute in a sc mutant background or in a $s c^{+}$ background (Fig. 2C, Fig. S3E-P). The 2036-bp $18 C 05$ region is located within the 5-kb candidate region identified with ac-sc structural mutations (Fig. 2C), suggesting that $18 C 05$ is a good candidate region for hypandrial bristle evolution. 
To test whether loss of hypandrial bristles in D. santomea resulted from changes(s) in the $18 C 05$ cis-regulatory region, we assayed whether orthologous $18 C 05$ regions from D. melanogaster, $D$. yakuba and $D$. santomea driving a $s c$ coding region could rescue hypandrial bristles in a $D$. melanogaster sc mutant (Table S7-8). The D. melanogaster $18 C 05$ enhancer rescued two bristles in both $s c^{29}$ and $s c^{M 6}$ mutant backgrounds, indicating that this construct mimics normal levels of $s c$ expression. The D. yakuba $18 C 05$ enhancer rescued on average 2 hypandrial bristles in $s c^{M 6}$ and 0.5 bristles in $s c^{29}$ whereas the $D$. santomea $18 C 05$ enhancer rescued significantly fewer bristles (1.1 in $s c^{M 6}$ and 0 bristles in $s c^{29}$, Fig. 3). For another measure of $18 C 05$ enhancer activity, we compared the ability of enhancer-GAL4 constructs containing the $18 C 05$ region from D. melanogaster, D. yakuba or $D$. santomea to induce extra bristles in sc mutants using the UAS-GAL4 system with UAS-sc. In this assay the $D$. santomea $18 C 05$ region also induced fewer bristles than the corresponding $D$. yakuba region (Fig. 3, GLM-Quasi-Poisson, $\mathrm{F}(19,509)=161.7, \mathrm{p}<10^{-5}$ for $s c^{29} ; \mathrm{F}(19,415)=125.9$, $\mathrm{p}<10^{-5}$ for $\left.s c^{M 6}\right)$. Together, these results suggest that changes(s) within $18 C 05$ contributed to hypandrial bristle evolution in D. santomea.

To narrow down the region responsible for hypandrial bristle loss, we dissected the $18 \mathrm{C} 05$ element from D. melanogaster, D. yakuba and D. santomea into smaller overlapping pieces and quantified their ability to produce hypandrial bristles with the GAL4 rescue experiment. For all three species we found that smaller segments rescued significantly fewer bristles than the corresponding full region (Fig. S4-5). Thus, transcription factor binding sites scattered throughout the entire $\sim 2 \mathrm{~kb}$ of the $18 C 05$ element are required to drive full expression in the hypandrial bristle region.

Sequence alignment of the $18 C 05$ region from multiple species revealed 11 substitutions and one indel that are fixed and uniquely derived in D. santomea. Among them, seven substitutions altered sites that are otherwise conserved in the D. melanogaster subgroup (Fig. S6-7). We tested the effect of these seven $D$. santomea-specific nucleotide changes by introducing them one at a time or all together, into either a $D$. yakuba $18 C 05$ enhancer or into the inferred ancestral enhancer driving sc expression (Tables S7-11). The ancestral $18 C 05$ sequence was resurrected by reverting the $D$. santomea-specific and $D$. yakuba-specific mutations to their ancestral states and it produced the same number of bristles as the D. yakuba construct (Fig. 3, Fig. S8). Four substitutions (G869A, T970A, T1008C and T1482C) had no effect, whether in the D. yakuba or in the ancestral background (GLM-Quasi-Poisson, $\mathrm{p}>0.6$ ). Three substitutions ( $T 1429 G, A 1507 G$ and $T 1775 G)$ decreased the number of rescued bristles in both the $D$. yakuba and the ancestral sequence, and these effects were highly significant, except for $A 1507 G$ in the D. yakuba background, which was slightly above statistical threshold (using the most stringent correction method) (Fig. 3). These results are consistent with analysis of smaller pieces of $18 C 05$ and of $18 C 05$ chimeric constructs containing DNA fragments from D. yakuba and D. santomea (Tables S711 , Fig. S8). When combined into the D. yakuba background, the seven $D$. santomea-specific substitutions rescued the same number of bristles as the D. santomea $18 C 05$ construct (Fig. 3, Fig. S8, GLM-Quasi-Poisson, $\mathrm{p}>0.9$ in $s c^{M 6}$ ). We conclude that at least three fixed substitutions within a 350-bp region located $49 \mathrm{~kb}$ away from $s c$ contribute to the reduction in hypandrial bristle number in $D$. santomea.

Analysis of 18C05-GAL4 and 18C05-GFP reporter constructs revealed that the $18 C 05$ region drives expression not only in male genital discs [19] but also in male developing forelegs in the presumptive sex comb region [19b] where $s c$ is broadly expressed (Fig. 4A-F). Sex combs are sensory organs used for grasping the female during copulation [20]. They differ in bristle number between $D$. santomea and D. yakuba (Fig. S9), and the difference maps to the X chromosome [21], where sc is located. These results prompted us to test whether the mutations contributing to hypandrial bristle evolution also affect sex combs. Significantly more GFP-positive cells were detected in the first tarsal segment at $5 \mathrm{~h}$ after puparium formation (APF) with $18 C 05 y a k T 1775 \mathrm{G}$-GFP than with $18 C 05 y a k$-GFP (GLM-Poisson, Chi-squared $(20,2)$ deviance $=9.75, \mathrm{p}=0.033)$, suggesting that $T 1775 G$ increases $s c$ expression in the first tarsal segment. Sex comb tooth number was reduced in $s c^{M 6}$ and $s c^{6}$ mutants and 
significantly rescued with several $18 C 05$-sc constructs (Fig. 4J-K). Analysis of $s c^{M 6}$ and $s c^{6}$ mutants rescued with the yak18C05-sc constructs containing the D. santomea-specific substitutions showed that $T 1429 \mathrm{G}$ and $T 1507 \mathrm{G}$ have no effect and that $T 1775 \mathrm{G}$ increases the number of sex comb teeth (Fig. 4J$\mathrm{K})$. We conclude that the $T 1775 G$ substitution contributes to both the increase in sex comb tooth number and the loss of hypandrial bristles.

A bioinformatics search revealed that the $T 1775 G$ substitution is predicted to alter a binding site for the Hox protein Abdominal-B (Abd-B) (Table S12). $A b d-B$ is expressed only in the posterior part of the fly, where it directs the development of posterior-specific structures such as the genitalia [22]. We found that reducing $A b d-B$ expression, using either genetic mutations or RNA interference, resulted in loss of hypandrial bristles (Fig. S10), indicating that normal levels of $A b d-B$ expression are required for hypandrial bristle development. Electrophoretic mobility shift assays showed that Abd-B proteins bind more strongly to a 54-bp fragment of the $18 C 05$ sequence containing the D. yakuba-specific T at position 1775 than the D. santomea-specific G at this position (Fig. S11). These results are consistent with the hypothesis that the $T 1775 G$ substitution decreases ABD-B binding, contributing to reduction in $s c$ expression levels, and ultimately reducing the number of hypandrial bristles. Since $A b d-B$ is not expressed in developing legs, $T 1775 G$ is expected to affect binding of other factors to increase sex comb tooth number. Overall, our study suggests that $T 1775 G$ alters overlapping binding sites for distinct factors in the leg and the genitalia (Fig. 4K). All our analyses of the effects of individual substitutions have been carried out in D. melanogaster background. It is thus possible that the $18 C 05$ enhancer represents only part of the effect of the $s c$ locus on bristle divergence.

Intriguingly, the two organs affected by substitution $T 1775 G$ - hypandrial bristles and sex combs - may both aid the male to position himself on top of the female during copulation [20,23]. Genitals are the most rapidly evolving organs in animals with internal fertilization [24]. To our knowledge, only two other mutations contributing to the evolution of genital anatomy are known. First, a 61-kb-deletion of a cis-regulatory region of the androgen receptor (AR) gene in humans is associated with loss of keratinized penile spines in humans compared to chimpanzees [25]. Second, an amino acid change in the nath10 acetyltransferase gene which probably appeared recently in laboratory strains of the nematode $C$. elegans, alters morphology in the presence of some mutations but not in a wild-type genetic background [10]. Both mutations appear to be pleiotropic: the $A R$ deletion is associated with loss of facial vibrissae in humans and the nath 10 mutation affects egg and sperm production as well. The paucity of known mutations responsible for genital evolution makes it currently difficult to propose general rules for the causes of rapid genital evolution. Our results are reminiscent of Mayr's pleiotropy hypothesis [26], which posits that certain characters may evolve arbitrarily as a result of selection on other traits due to pleiotropic mutations. In our case, whether the evolutionary change in sex comb tooth number or in genital bristle number has any effect on fitness is unknown.

We report here the first case of a cis-regulatory substitution between species with pleiotropic effects. Given the large number of bristle types regulated by $s c$ ( $>100$ in adult flies), it is possible that no cis-regulatory mutation in $s c$ can affect only one bristle type. Our results challenge the idea that cisregulatory enhancers are strict tissue-specific modules underlying evolutionary changes in targeted traits [27]. Even though cis-regulatory mutations may affect several tissues, it is probable that they still tend to be less pleiotropic than coding changes. Our results are thus compatible with the idea that cisregulatory changes tend to have fewer pleiotropic effects than coding changes on average. Enhancer sequences evolve rapidly, with rapid turn over of individual binding sites while maintaining transcriptional output over millions of years by compensatory mutations [28]. Since pleiotropic mutations can have deleterious off-target effects, we propose that evolution of pleiotropic sites within enhancers should trigger the subsequent selection of compensatory mutations in cis, thus contributing to rapid divergence of cis-regulatory sequences. Overall, our results suggest that pleiotropic cisregulatory mutations may play a more important role in evolution than previously thought. 
Data and materials availability: Sequences were deposited into GenBank (accession numbers MG460736-MG460765). Source data for Bristle Number and QTL mapping analysis are available as Auxiliary Supplemental Files.

\section{Supplemental Information includes:}

Figures S1-S11

Tables S1-S13

Supplemental References (29-63)

\section{ACKNOWLEDGEMENTS}

We thank the Tucson Drosophila Species Stock Center, the VDRC, Kyoto and Bloomington Stock Centers for flies. We thank São Tomé authorities for allowing us to collect flies. We thank S. Picard for help with MSG, E. Sánchez-Herrero for $A b d-B$ flies, F. Schweisguth for GFP-sc flies, J. Selegue and S.B. Carroll for the $A b d-B$ construct, L. Pintard and N. Joly for help in protein purification, R. Mann, S. Feng and G. Rice for EMSA suggestions, F. Mallard and T. Tully for advices on the statistical analyses, J. L. Villanueva-Cañas for help with JASPAR. We thank Q.D. Tran, M. Notin, V. Ludger, A. Matamoro-Vidal, C. Nobre, G. Verebes, S. El Ouisi, A. La, F. Foutel-Rodier, C. Guillard-Sirieix and A. Aydogan for their contributions and C. Desplan, D. Petrov, B. Prud'homme, A. Martin and J.A. Lepesant for comments on the manuscript. We acknowledge the ImagoSeine core facility of the Institut Jacques Monod, member of IBiSA and France-BioImaging (ANR-10-INBS-04) infrastructures. We thank the Courtier-Orgogozo team for providing a stimulating environment and technical support.

\section{FUNDING}

The research leading to this paper has received funding from the European Research Council under the European Community's Seventh Framework Program (FP7/2007-2013 Grant Agreement no. 337579) to VCO, from the labex "Who am I?" (ANR-11-LABX-0071) funded by the French government through grant no. ANR-11-IDEX-0005-02 to AEP and from NIH (1R01GM121750) to DRM.

\section{AUTHOR CONTRIBUTIONS}

J.R.D. found that D. santomea lacks hypandrial bristles and that the trait difference is X-linked, D.L.S. genotyped flies with MSG, A.Y., I.N. and V.C.O. performed the QTL mapping experiment, D.R.M. made the D. santomea-D. melanogaster hybrids, I.N. dissected them, O.N. did all other fly crosses and dissected them, O.N., I.N., R.S. and A.E.P. phenotyped $>3000$ males for hypandrial bristles, O.N. phenotyped all other bristles, O.N. and M.L. did EMSA, O.N. and I.N. constructed the plasmids, O.N. performed immunostainings and microscopy, A.E.P. performed all statistical analyses with feedback from O.N. and M.L., D.R.M. collected wild flies, V.C.O. supervised research, performed bioinformatics sequence analysis and wrote the paper with O.N. All authors provided feedback on the text.

\section{DECLARATION OF INTEREST}

The authors declare no competing interests. 


\section{REFERENCES}

1. Darwin, C. (1859). On the origin of species by means of natural selection, or the preservation of favoured races in the struggle for life.

2. Saltz, J.B., Hessel, F.C., and Kelly, M.W. (2017). Trait Correlations in the Genomics Era. Trends Ecol. Evol. (Amst.) $32,279-290$.

3. Paaby, A.B., and Rockman, M.V. (2013). The many faces of pleiotropy. Trends in Genetics 29, 66-73.

4. Fisher, R.A. (1930). The genetical theory of natural selection (Oxford: Clarendon).

5. Orr, H.A. (2000). Adaptation and the cost of complexity. Evolution 54, 13-20.

6. Wagner, G.P., and Zhang, J. (2011). The pleiotropic structure of the genotype-phenotype map: the evolvability of complex organisms. Nature Reviews Genetics 12, 204-213.

7. Stearns, F.W. (2010). One Hundred Years of Pleiotropy: A Retrospective. Genetics 186, 767-773.

8. Lonfat, N., Montavon, T., Darbellay, F., Gitto, S., and Duboule, D. (2014). Convergent evolution of complex regulatory landscapes and pleiotropy at Hox loci. Science 346, 1004-1006.

9. Preger-Ben Noon, E., Sabarís, G., Ortiz, D.M., Sager, J., Liebowitz, A., Stern, D.L., and Frankel, N. (2018).

Comprehensive Analysis of a cis -Regulatory Region Reveals Pleiotropy in Enhancer Function. Cell Reports 22, 30213031.

10. Duveau, F., and Félix, M.-A. (2012). Role of pleiotropy in the evolution of a cryptic developmental variation in Caenorhabditis elegans. PLoS Biol. 10, e1001230.

11. Chang, S.H., Jobling, S., Brennan, K., and Headon, D.J. (2009). Enhanced Edar Signalling Has Pleiotropic Effects on Craniofacial and Cutaneous Glands. PLOS ONE 4, e7591.

12. Kent, C.F., Daskalchuk, T., Cook, L., Sokolowski, M.B., and Greenspan, R.J. (2009). The Drosophila foraging Gene Mediates Adult Plasticity and Gene-Environment Interactions in Behaviour, Metabolites, and Gene Expression in Response to Food Deprivation. PLOS Genetics 5, e1000609.

12b. Endler, L., Gibert, J. M., Nolte, V., \& Schlötterer, C. (2018). Pleiotropic effects of regulatory variation in tan result in correlation of two pigmentation traits in Drosophila melanogaster. Molecular ecology

13. Wittkopp, P.J., Haerum, B.K., and Clark, A.G. (2008). Regulatory changes underlying expression differences within and between Drosophila species. Nature Genetics 40, 346-350.

14. Turissini, D.A., and Matute, D.R. (2017). Fine scale mapping of genomic introgressions within the Drosophila yakuba clade. PLoS Genetics 13, e1006971.

15. Lachaise, D., Harry, M., Solignac, M., Lemeunier, F., Bénassi, V., and Cariou, M.L. (2000). Evolutionary novelties in islands: Drosophila santomea, a new melanogaster sister species from São Tomé. Proc. Biol. Sci. 267, 1487-1495.

16. Simpson, P., Woehl, R., and Usui, K. (1999). The development and evolution of bristle patterns in Diptera. Development 126, 1349-1364.

17. Gómez-Skarmeta, J.L., Rodríguez, I., Martínez, C., Culí, J., Ferrés-Marcó, D., Beamonte, D., and Modolell, J. (1995). Cis-regulation of achaete and scute: shared enhancer-like elements drive their coexpression in proneural clusters of the imaginal discs. Genes Dev 9, 1869-1882. 
18. Marcellini, S., Gibert, J.-M., and Simpson, P. (2005). achaete, but not scute, is dispensable for the peripheral nervous system of Drosophila. Dev. Biol. 285, 545-553.

19. Jory, A., Estella, C., Giorgianni, M.W., Slattery, M., Laverty, T.R., Rubin, G.M., and Mann, R.S. (2012). A Survey of 6,300 Genomic Fragments for cis-Regulatory Activity in the Imaginal Discs of Drosophila melanogaster. Cell Reports $2,1014-1024$.

19b. Tanaka, K., Barmina, O., Sanders, L. E., Arbeitman, M. N., \& Kopp, A. (2011). Evolution of sex-specific traits through changes in HOX-dependent doublesex expression. PLoS biology, 9(8), e1001131.

20. Ng, C.S., and Kopp, A. (2008). Sex combs are important for male mating success in Drosophila melanogaster. Behavior genetics 38,195 .

21. Coyne, J.A., Elwyn, S., Kim, S.Y., and Llopart, A. (2004). Genetic studies of two sister species in the Drosophila melanogaster subgroup, D. yakuba and D. santomea. Genetics Research 84, 11-26.

22. Foronda, D., Estrada, B., de Navas, L., and Sánchez-Herrero, E. (2006). Requirement of Abdominal-A and Abdominal$\mathrm{B}$ in the developing genitalia of Drosophila breaks the posterior downregulation rule. Development 133, 117-127.

23. Hurtado-Gonzales, J.L., Gallaher, W., Warner, A., and Polak, M. (2015). Microscale Laser Surgery Demonstrates the Grasping Function of the Male Sex Combs in Drosophila melanogaster and Drosophila bipectinata. Ethology 121, 4556.

24. Eberhard, W.G. (1988). Sexual Selection and Animal Genitalia (Harvard University Press).

25. McLean, C.Y., Reno, P.L., Pollen, A.A., Bassan, A.I., Capellini, T.D., Guenther, C., Indjeian, V.B., Lim, X., Menke, D.B., Schaar, B.T., et al. (2011). Human-specific loss of regulatory DNA and the evolution of human-specific traits. Nature 471, 216-219.

26. Mayr, E. (1963). Animal species and evolution (Harvard University Press).

27. Carroll, S.B. (2008). Evo-devo and an expanding evolutionary synthesis: a genetic theory of morphological evolution. Cell 134, 25-36.

28. Cheng, Y., Ma, Z., Kim, B.-H., Wu, W., Cayting, P., Boyle, A.P., Sundaram, V., Xing, X., Dogan, N., Li, J., et al. (2014). Principles of regulatory information conservation between mouse and human. Nature 515, 371. 


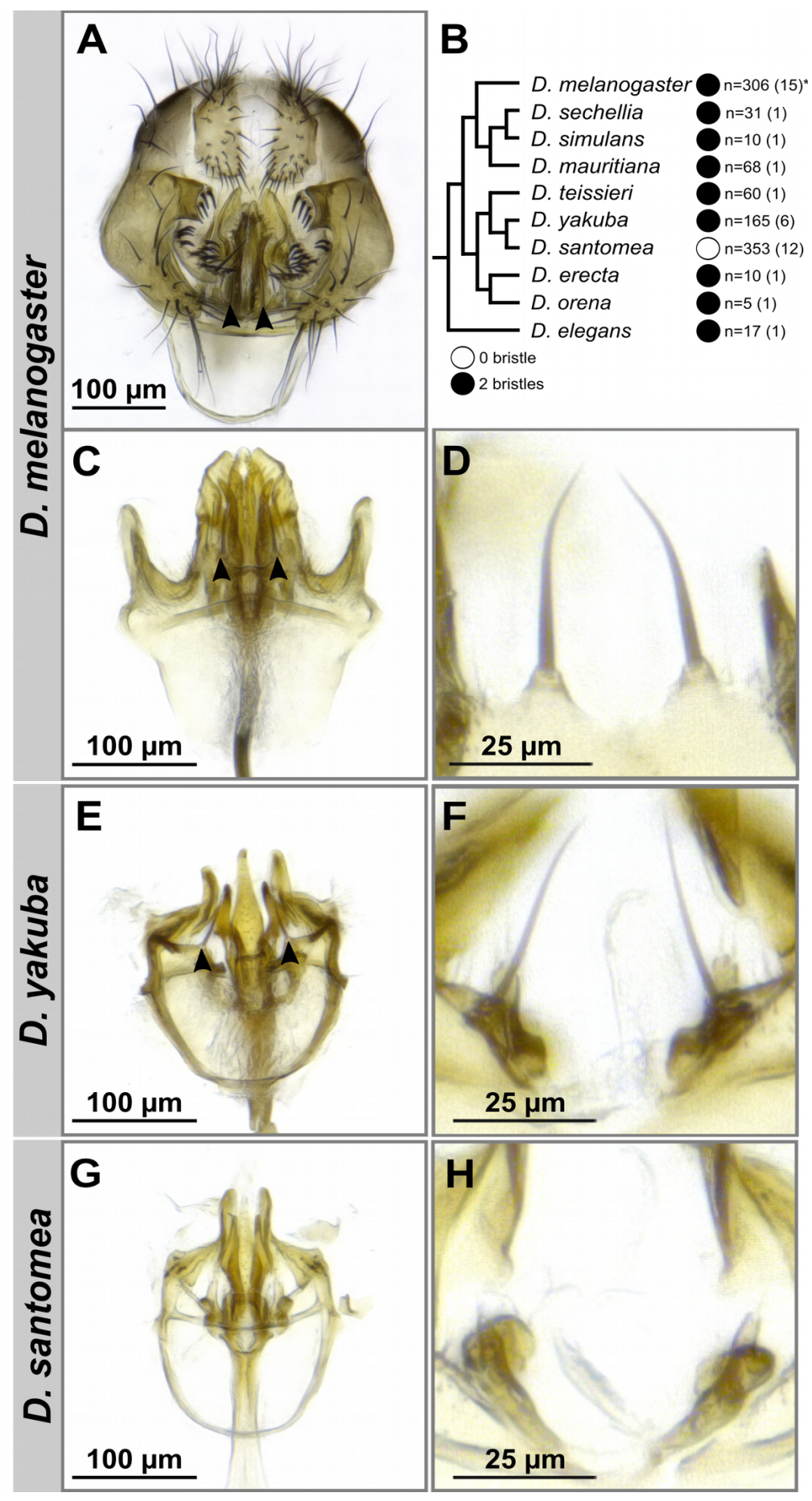

Fig. 1. D. santomea lost hypandrial bristles.

(A) Drosophila melanogaster male genitalia. (B) Phylogeny of the Drosophila melanogaster species subgroup. All species of have two hypandrial bristles (black circles) except Drosophila santomea, which lacks hypandrial bristles (white circle). n: number of scored males, with the number of scored strains in parentheses. Asterisk indicates that 4 males out of 306 had three hypandrial bristles. (C-H) Light microscope preparations of ventral genitalia (C,E,G) and hypandrial bristles (D,F,H) in $D$. melanogaster (C-D), D. yakuba (E-F) and D. santomea (G-H). Hypandrial bristles are indicated with arrowheads on $\mathrm{A}, \mathrm{C}$ and $\mathrm{E}$. 


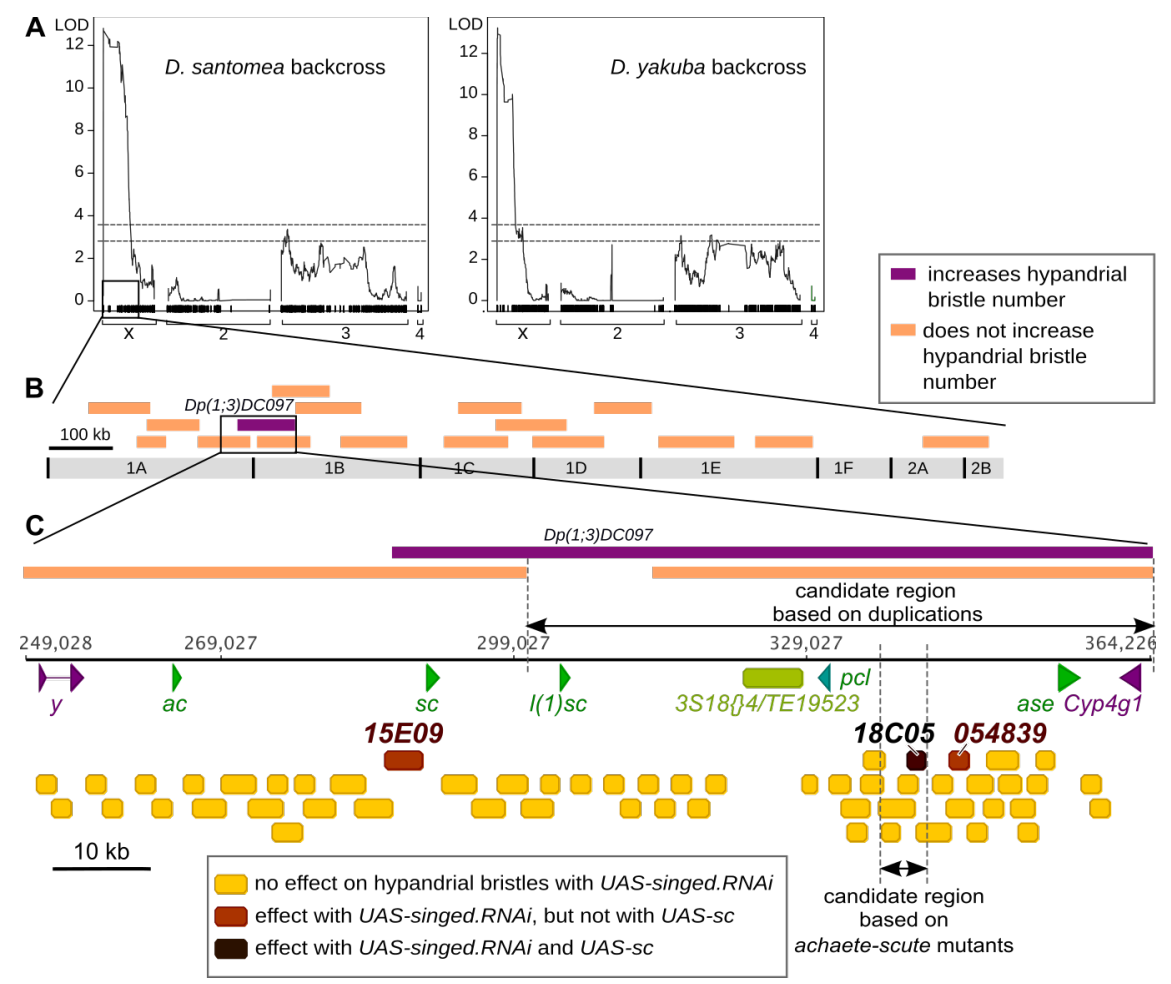

Fig. 2. Mapping of the cis-regulatory element involved in hypandrial bristle evolution. backcross (right). On the y-axis are the LOD profiles from a Haley-Knott regression analysis. The xaxis represents physical map position in the D. yakuba genome. Ticks represent recombination informative markers. Dotted lines represent the 1\% (top) and 5\% (bottom) significance thresholds. (B) Schematic representation of the left tip of chromosome X and of 19 duplicated fragments of chromosome $\mathrm{X}$ that were tested for their effect on hypandrial bristle number in $\mathrm{D}$. santomea- $D$. melanogaster hybrid males. All duplications had no significant effect (orange) except $D p(1 ; 3) D C 097$ (purple), which significantly increased hypandrial bristle number. (C) Genomic organization of the $A S$ $C$ locus in D. melanogaster. Arrows indicate the coding regions of yellow $(y)$, achaete $(a)$, scute $(s c)$, lethal of scute (l(1)sc), pepsinogen-like ( $\mathrm{pcl}$ ), asense (ase) and cytochrome P450-4gl(Cyp4gl) genes. The light green box represents the insertion of a $3 S 18\{\} 4 / T F 9523$ natural transposable element. Boxes indicate cis-regulatory elements whose corresponding GAL4 reporter lines have been tested. Expression of $U A S$-singed.RNAi with 52 GAL4 lines (yellow boxes) has no effect while it results in singed hypandrium bristles with 15E09-, 18C05- and 054839-GAL4. Extra hypandrial bristles are found with $U A S$-sc and 18C05-GAL4 (dark brown box) but not with 15E09- and 054839-GAL4 (light brown boxes). 

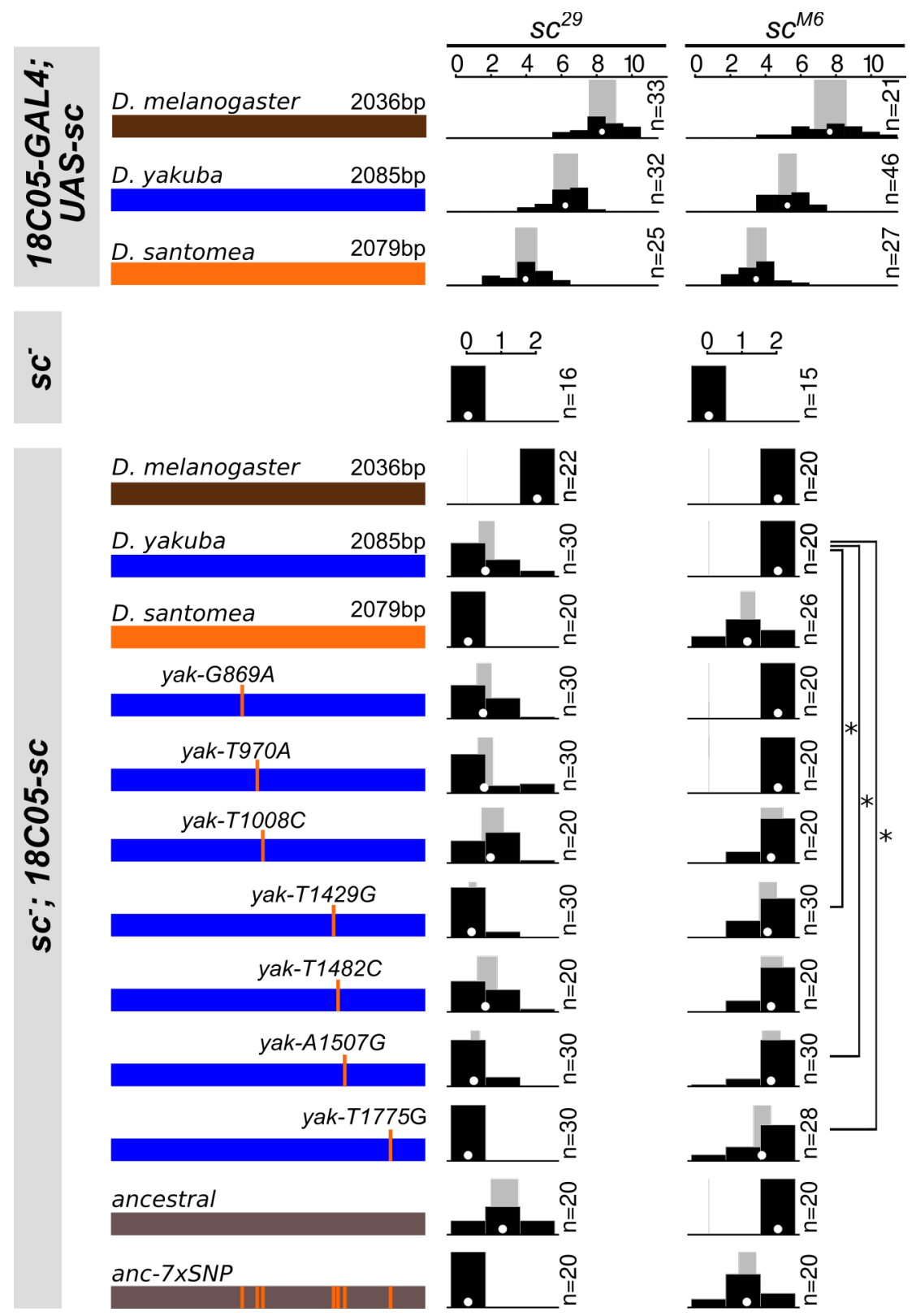
bristles.

Rescue of the hypandrial bristle loss of $s c^{29}$ (left column) and $s c^{M 6}$ (right column) D. melanogaster mutants by expression of either GAL4 with $U A S-s c$ or $s c$ driven by $18 C 05$ sequences from $D$. melanogaster (brown), D. yakuba (blue) and D. santomea (orange). Seven D. santomea-specific substitutions (vertical orange bars) were introduced into either the D. yakuba region (blue) or the ancestrally reconstructed $18 C 05$ region (grey). Distribution of hypandrial bristle number (black histogram), together with mean (white dot) and 95\% confidence interval (grey rectangle) from a fitted GLM Quasi-Poisson model are shown for each genotype. Note that for a given rescue construct, 18C05-GAL4 UAS-SC produces more hypandrial bristles than 18C05-sc, probably due to the amplification of gene expression caused by the GAL4/UAS system. n: number of scored individuals. *: $\mathrm{p}<0.05$ 

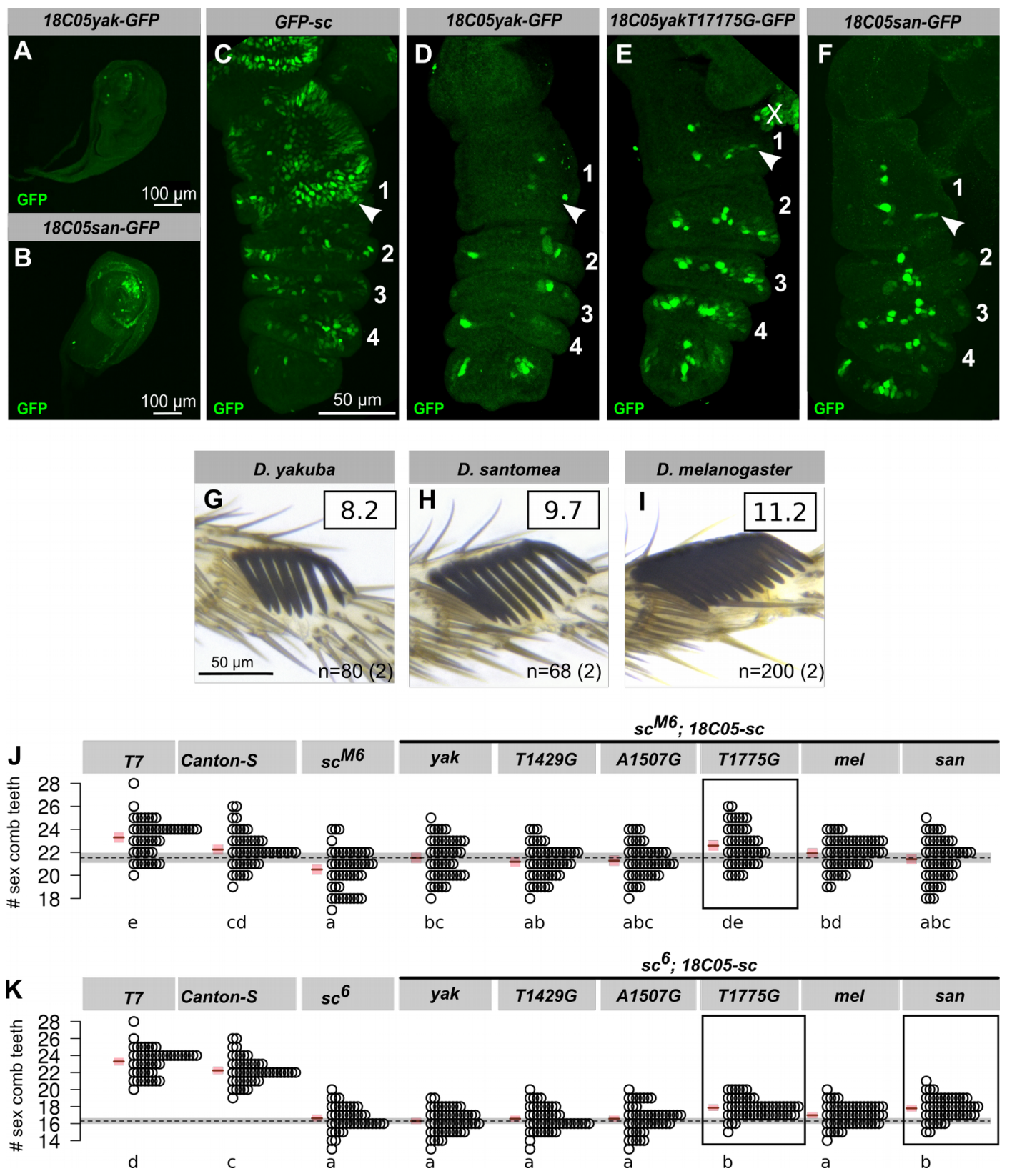
(A-F) $18 C 05$ drives expressionGFP staining in T1 leg discs of late L3 larvae (A-B) and in 5h APF pupal legs (C-F) in D. melanogaster. Genotype is indicated on top of each panel. Tarsal segments are numbered. Arrowheads point to the presumptive sex comb regions. " $\mathrm{X}$ " indicates non-leg tissue. late L3 larvae containing either $18 C 05 y a k u b a-G F P(\mathrm{~A}-\mathrm{C})$ or $18 C 05$ santomea-GFP (D-F) transgenes. GFP is labeled in green (A, C, D, F), DNA is shown in blue (B, C, E, F). (G-I) Leg sex comb in D. yakuba $(\mathrm{G})$, D. santomea $(\mathrm{H})$ and D. melanogaster $(\mathrm{I})$. Average sex comb tooth numbers per leg are shown in squares. n: number of scored individuals, with the number of scored strains in parentheses. (J-K) Sex comb tooth number in wild-type (T7 and Canton-S), $s c^{M 6}(\mathrm{~J})$ and $s c^{6}(\mathrm{~K}) s c^{M 6-}$ mutants and $s c^{M 6}(\mathrm{~J})$ and $s c^{6}(\mathrm{~K})$ and $s c^{M 6}$ mutants rescued with different 18C05-sc constructs, . Each circle represents one male raised at $25^{\circ} \mathrm{C}$. Mean (brown line) and $95 \%$ confidence interval (pink rectangle) from a fitted GLM Quasi-Poisson model are shown. Letters indicate the results of all-pairwise comparisons after HolmBonferroni correction. Two genotypes are significantly different from each other $(p<0.05)$ when they do not share a letter. For easier comparison, the horizontal dashed line and the surrounding grey line indicate the mean and 95\% confidence interval for $s c^{-} ; 18 C 05 y a k$-sc. Transgenic constructs with sex comb tooth number significantly different from 18C05ak-sc are shown in boxes in J-K. On average $D$. santomea males have about 1 extra tooth per sex comb compared to $D$. yakuba (G$\mathrm{H}$ ) and $35 \%$ of this difference has been attributed to the $\mathrm{X}$ chromosome [21]. The substitution $T 1775 G$ produces on average 0.5 extra sex comb tooth per leg, which is more than expected. It is possible that the D. melanogaster background, where all our rescue constructs were tested, amplifies the effect of the tested substitutions, especially since $D$. melanogaster males have more sex comb teeth than $D$. santomea or D. yakuba. 
STAR*METHODS

\section{CONTACT FOR REAGENT AND RESOURCE SHARING}

Further information and requests for resources and reagents should be directed to and will be fulfilled by the Lead Contact, Virginie Courtier-Orgogozo (virginie.courtier@normalesup.org).

\section{EXPERIMENTAL MODEL AND SUBJECT DETAILS}

The origin of all the fly strains used can be found in Tables S1,3,5-6. All flies were cultured on standard cornmeal-agar medium in uncrowded conditions at $25^{\circ} \mathrm{C}$ unless stated. We used Canton-S as a wildtype $D$. melanogaster strain. Transgenic constructs were integrated into the attP2 landing site in $D$. melanogaster $w^{1118}$ by BestGene Inc. Hybrid males between D. yakuba and D. santomea were obtained by collecting 20 virgin females with 20 males from each stocks and crossing them reciprocally in both directions. At least 10 such crosses were made and flipped every 4-5 days for several weeks. For QTL mapping, D. yakuba yellow [1] virgin females were crossed en masse to D. santomea SYN2005 males to generate F1 hybrid females, which were subsequently backcrossed, separately, to both parental strains. Genitalia of backcross males were isolated for dissection and the remaining carcass was stored at $-20{ }^{\circ} \mathrm{C}$ for subsequent sequencing library preparation.

\section{METHOD DETAILS}

Genotyping of backcross males for QTL mapping

The carcass of each male was crushed in a 1.5-ml Eppendorf tube with a manual pestle in $180 \mu 1$ of Qiagen Tissue Lysis buffer. DNA of individual flies was extracted using Qiagen DNeasy Blood \& Tissue extraction kit (cat \#69506). A Multiplexed Shotgun Genotyping sequencing library was made from $189 \mathrm{D}$. santomea backcross males and for $181 \mathrm{D}$. yakuba backcross males as described previously [29]. The list of barcodes used in this study are provided in Supplemental Data File 1, within the names of the individuals that were sequenced. D. yakuba and D. santomea parental genome sequences were generated by updating the $D$. yakuba genome sequence dyak-4-chromosome-r1.3.fasta with Illumina paired-end reads from D. yakuba yellow [1] and D. santomea SYN2005 (sequenced by BGI) using the msgUpdateParentals.pl function of the MSG software package. The resulting updated genome files are dsan-all-chromosome-yak1.3-r1.0.fasta.msg.updated.fasta and dyak-4-chromosomer1.3.fasta.msg.updated.fasta. Ancestry was estimated for all backcross progeny using MSG software (github.com/YourePrettyGood/msg). Ancestry files were reduced to only those markers informative for recombination events using the script pull_thin_tsv.py (github.com/dstern/pull_thin). Markers were considered informative when the conditional probability of being homozygous differed by more than 0.05 from their neighboring markers.

QTL mapping QTL mapping was performed using the R/qtl package version 1.4 [30,31]. The thinned posterior genotype probabilities were imported into $\mathrm{R} / \mathrm{qtl}$ using the $\mathrm{R}$ function read.cross.msg.1.5.R (github.com/dstern/read_cross_msg). QTL mapping was performed independently on each backcross 
population. We performed genome scans with a single QTL model ("scanone") using the Haley-Knott regression method [32] performs well with genotype information at a large number of positions along the genome. The genome-wide 5\% and 1\% significance levels were determined using 1,000 permutations. One QTL peak above the 1\% significance level was found for both backcrosses. To check for additional QTL, we built a QTL model with this single QTL using the "fitqtl" function and scanned for additional QTL using the "addqtl" function. A second QTL was found on chromosome 3 for both backcrosses. When introduced into a new multiple QTL model, refined and fitted to account for possible interactions, a third significant QTL was found. Based on the full three-QTL model, no additional significant QTL were found with the function "addqtl": the highest LOD score for a fourth QTL reached only 1.8 and 1.2 for the D. yakuba backcross and the D. santomea backcross, respectively. Various three-QTL models with different interactions between loci were assessed. Positive significant interaction was detected between the QTL on chromosome 1 and both QTLs on chromosome 3. The interaction between the two QTLs on chromosome 3 was not significant. For the three-QTL model with interactions between the QTL on chromosome 1 and both QTLs on chromosome 3 , we computed the LOD score of the full model and the estimated effects of each locus. The 2-LOD intervals were calculated using the "lodint" function with parameter drop of 2. Analysis of F1 hybrid males is consistent with a large effect of the $\mathrm{X}$ chromosome on hypandrial bristle number: male F1 hybrids carrying a $D$. yakuba X chromosome have on average 1.9 hypandrial bristles $(\mathrm{n}=34)$ while reciprocal hybrid males possessing the $D$. santomea $X$ chromosome have none $(n=29)$ (Table S2). Note that few informative markers are found on the right arm of chromosome 2, suggesting the presence of an inversion between parental lines. In both backcrosses the large-effect QTL is estimated to cause a decrease of $0.9 \pm 0.1$ bristles between a $D$. yakuba hemizygote and a $D$. santomea hemizygote male (Data S1). The QTL peak is at position 46,886 and 221,928 for the D. santomea and D. yakuba backcross, respectively. The $A S-C$ locus is at position 179,000-290,000.

\section{Duplication Mapping in D. santomea-D. melanogaster hybrids}

We used a set of $D$. melanogaster duplication lines to test overlapping parts of chromosome $\mathrm{X}$ for their effect on hypandrial bristle number [33]. Each line contains a fragment of the chromosome X inserted into the same attP docking site on chromosome 3L using ФC31 integrase, allowing direct comparison between fragments. Each duplication was used to screen for complementation of the loss of function allele(s) from D. santomea. We exploited the fact that rare D. santomea-D. melanogaster hybrid males can be produced by crossing $D$. melanogaster females carrying a compound $\mathrm{X}$ chromosome with $D$. santomea males [34]. The resulting hybrid males carry a $D$. santomea $\mathrm{X}$ chromosome. We first created a D. melanogaster stock whose genotype is TM3, Sb [1] Ser[1]/Nup98-96[339] by crossing Nup9896[339]/TM3, $S b$ [1] with $D f(3 R) D 605 / T M 3, S b$ [1] Ser [1]. We then performed three successive crosses at room temperature in glass vials: (a) C(1)RM, y[1] w [1]f[1] ; +/+ $\times+/+$; TM3, Sb [1] Ser [1]/Nup9896[339], (b) C(1)RM, y[1] w[1]f[1] ; TM3, Sb[1] $\operatorname{Ser}[1] /+\times+/ Y ; \operatorname{Dp}(1,3) / D p(1,3)$, (c) C(1)RM, y[1] $w[1] f[1] ; T M 3, S b[1] \operatorname{Ser}[1] / D p(1,3) D$. melanogaster females $\times D$. santomea males. The same procedure was followed for 21 duplication lines and progeny was obtained for 17 of them. Hybrid males from the last cross were sorted in two pools, the $\left[\mathrm{S} b^{-}, \mathrm{Ser}\right]$ males who carried the duplication and the $\left[\mathrm{Sb}^{+}, \mathrm{Ser}^{+}\right]$males which were used as controls which carried no duplication but the balancer chromosome TM3 Sb[1] Ser[1]. In D. melanogaster/D. santomea hybrids, dominant markers are not always fully penetrant. A few progeny males exhibited $\left[\mathrm{Sb}^{+}, \mathrm{Ser}^{-}\right]$or $\left[\mathrm{Sb}^{-}, \mathrm{Ser}^{+}\right]$phenotypes; they were considered as control individuals carrying the balancer chromosome TM3, Sb[1] Ser [1]. Males were stored in ethanol until dissection. Duplication mapping narrowed down the causal region to a $84.6 \mathrm{~kb}$ region (DC097) of the achaete-scute complex $(A S-C)$ (Fig. 2.B-C, Table S2, GLM-Poisson, $\left.\operatorname{Chisq}(17,478)=398.44, \mathrm{p}=10^{-4}\right)$. 


\section{Examination of Hypandrial Bristle Phenotypes}

Male genitalia were cut with forceps and then hypandria were dissected with fine needles or forceps Dumont \#5 (112525-20, Phymep) in a drop of 1x PBS. For D. melanogaster in order to see the hypandrial bristles better we removed the aedeagus by holding the aedeagal apodem with forceps and gently pushing the hypandrium upwards with an other forceps until it separated. Hypandria were mounted in DMHF (Dimethyl Hydantoin Formaldehyde, Entomopraxis). Before dissection, males were sometimes stored at $-20^{\circ} \mathrm{C}$ in empty Eppendorf tubes or in glycerol:acetate:ethanol $(1: 1: 3)$ solution. For analysis of non-hypandrial bristles, males were stored at $-20^{\circ} \mathrm{C}$ in glycerol:acetate:ethanol $(1: 1: 3)$ solution. We never stored these males in empty tubes because we found that such a storage procedure can break and remove external bristles (but, as far as we know, hypandrial bristles were not affected by such a procedure, maybe because hypandrial bristles are relatively internal and protected by the epandrium). Furthermore, we never observed a single socket devoid of shaft on the male hypandrium, indicating that hypandrial bristles cannot be accidentally cut or lost with our experimental protocol. 3D projection images of the preparations were taken at 500X magnification with the Keyence digital microscope VHX 2000 using optical zoom lens VH-Z20R/W.

\section{Examination of Other Bristles}

Since genitalia are the most rapidly evolving organs in animals with internal fertilization [32], we compared the number of genital bristles between two strains of $D$. yakuba and two strains of $D$. santomea. We found no difference between D. yakuba and D. santomea in any genital bristles except for anal plate and clasper bristles, where a slightly significant interspecific variation was detected (Fig. $\mathrm{S} 1$.). The loss of hypandrial bristles in $D$. santomea is thus the major change in genital bristles between D. santomea and D. yakuba. Genitalia were dissected in $1 \mathrm{X}$ PBS, hypandria were removed and the epandria were mounted in $99 \%$ glycerol. Gentle pressure was applied on the cover-slip with forceps to flatten the preparations in order to see all bristles. Pictures were taken at a 500X magnification with a digital microscope VHX 2000 (Keyence) using lens VH-Z20R/W. Bristles were counted on the images. For sex comb preparations, prothoracic legs were dissected at the coxa with forceps Dumont \#5 and were mounted in DMHF (Dimethyl Hydantoin Formaldehyde, Entomopraxis). Images of the sex combs were taken at 1000x magnification with the Keyence digital microscope as written above. Sex comb teeth were counted on the images with Image J [35]. .

\section{Analysis of scute coding sequence}

The scute coding sequence (CDS) of D. melanogaster iso-1 was retrieved from FlyBase. We blasted the updated genome sequences of D. yakuba yellow [1] and D. santomea SYN2005 (see above) with $D$. melanogaster scute coding region and retrieved only one locus in each species. The scute coding region was then annotated with Geneious and no intron was found, as in D. melanogaster.

\section{Screening as-GAL4 lines for expression in the hypandrium}

The as-GAL4 lines were ordered from VDRC and Bloomington Stock Center (Table S6). Two lines were not available (GMR 1509 and VT054822) so we created new transgenic lines for these regions, named GMR15X09-GAL4 and VT054822b-GAL4 (see below). Because screens are easier on adults than on genital discs, and also because the exact developmental stage and location of hypandrial bristle development are unknown [36], we decided to look for GAL4-triggered phenotypes in adult males. As a readout of GAL4 expression, we tested various UAS lines (UAS-mCD8-GFP, UAS-yellow in a yellow mutant background, UAS-sc.RNAi, UAS-achaete.RNAi, UAS-forked.RNAi, UAS-singed.RNAi) (lines are listed in Table $\mathrm{S} 1$ and S5) together with $D C$-GAL4, which drives expression in the dorso-central thoracic bristles [37]. To enhance RNAi potency we also used UAS-Dicer-2 (35). With UAS-mCD8GFP and UAS-yellow the change in fluorescence or color was hardly visible. The most penetrant bristle 
phenotype was obtained with UAS-Dicer-2 UAS-singed.RNAii $1^{05747}$ at $29^{\circ} \mathrm{C}$ (Table S5). Therefore this line was chosen for screening all the as-GAL4 constructs.

Five as-GAL4 males of each as-GAL4 line were crossed to five $D c r 2$; $U A S$-singed ${ }^{105747} . R N A i / C y O$ virgin females. Crosses were kept at $29{ }^{\circ} \mathrm{C}$. The non-curly males (Dcr2; UAS-singed ${ }^{105747} . R N A i /+$; + las-GAL4) were collected for dissection and kept at $-20{ }^{\circ} \mathrm{C}$. Hypandrium dissection and image acquisition were performed as indicated above. For each as-GAL4 line at least 5 genitalia were examined (Table S6).

To test whether the 15E09, $18 C 05$ and 054839 enhancer-GAL4 drive expression in the hypandrial bristle region in absence of $s c$, we crossed five $s c^{29}$; UAS-scute (III) females with five males of each respective GAL4 line, as well as five $s c^{\mathrm{Mb}} / F M 7$; UAS-scute (III) females with five males of each respective GAL4 line. Of the three GAL4 lines, only $18 C 05$ could induce hypandrial bristles with $U A S$ $s c$ in a $s c$ mutant background. The 18C05-GAL4 line produced approximately 10 bristles, where normally only two develop, which may reflect the amplification of gene expression that is inherent to the UAS-GAL4 system. These results suggest that only $18 C 05$ drives sufficiently strong expression in the hypandrial region to alter bristle patterning.

\section{Cloning of enhancers into pBPGUw and pBPSUw}

Enhancers were cloned into the GAL4 reporter vector pBPGUw using the same strategy as in $[37,38]$. Enhancer sequences were amplified by Phusion ${ }^{\circledR}$ High Fidelity Polymerase (New England Biolabs) in two steps reaction using the primers and templates listed in Table S7, S8 and S9. PCR products and vectors were purified by Nucleospin Gel and PCR Clean-Up Kit (Machery-Nagel). Clones were purified by E.Z.N.A. ${ }^{\circledR}$ Plasmid Mini Kit I (Omega Bio-tek). All GAL4 constructs were cloned using the Gateway ${ }^{\circledR}$ system (ThermoFisher Scientific). The enhancer fragments were first ligateded into KpnI and HindIII restriction enzyme site of the vector pENTR/D-TOPO (Addgene) (Table S8). Recombination into the destination vector pBPGUw was performed using LR clonase II enzyme mix (Invitrogen) and products were transformed into One Shot ${ }^{\circledR}$ TOP10 (Invitrogen) competent cells. Recombinant clones were selected by ampicillin resistance on Amp-LB plates $(100 \mu \mathrm{g} / \mathrm{ml})$

The pBPSUw vector was constructed by replacing the GAL4 cassette of pBPGUw by scute CDS. The scute $C D S$ was amplified from D. melanogaster iso- 1 with Scute-CDS-Rev and Scute-CDS-For primers and ligated into pGEM-T Easy (Promega). The $s c-C D S$ insert was cut out using $K p n \mathrm{I}$ and HindIII and cloned into KpnI and HindIII sites in pBPGUw, thus replacing GAL4. The vector was named pBPSUw where "S" stands for scute. $18 C 05$ sequences from D. melanogaster, D yakuba and D. santomea were cloned into pBPSUw and tested in rescuing hypandrial bristles in $s c$ mutants as written above. We found that $18 C 05$ from $D$. melanogaster rescued two hypandrial bristles in both $s c^{29}$ and $s c^{\mathrm{Mr}}$ mutants. D. santomea $18 C 05$ enhancer rescued fewer hypandrial bristles on average than the $D$. yakuba $18 C 05$ region (Fig. 3., bristle number for D. yakuba $18 C 05$ in $s c^{29}$ is significantly different from 0 (Exact-Poisson, $\mathrm{p}<10^{-16}$ ) and bristle number for $D$. santomea $18 C 05$ in $s c^{M 6}$ is significantly different from 2 (Exact-Poisson, $\mathrm{p}=0.0008)$ ).

The $18 C 05$ full length sequences were amplified by PCR from D. melanogaster iso-1(BL2057), D. melanogaster T-7, D. yakuba Ivory Coast and D. santomea SYN2005 with the primers listed in Table S7. The PCR products were cloned into $p B P S U w$ as described above. Three different $D$. melanogaster $18 C 05$ sequences were tested with $U A S-s c$ in the hypandrium in $s c^{29}$ and $s c^{M 6} . G M R-18 C 05$ (BL2057) was obtained from the Janelia Farm collection [38] and 18C05_BL2057 and 18C05_T7 were cloned in this study. Hypandrial bristle number was found to be significantly higher for GMR-18C05 than for 18C05_BL2057 and 18C05_T7 in both backgrounds (GLM-Quasi-Poisson, $\mathrm{F}(2,63)=16.88$, both $\mathrm{p}<$ $10^{-6}$ for $s c^{29} ; \mathrm{F}(2,58)=20.9, \mathrm{p}<10^{-10}$ and $\mathrm{p}<10-5$ for $\left.s c^{M 6}\right)$. The GMR-18C05 fragment is inserted in the expression vector $3^{\prime}-5^{\prime}$ compared to the $D$. melanogaster genome sequence. In contrast, the 18C05_BL2057 and 18C05_T7 are cloned 5'-3'. All the $18 C 05$ constructs we made were inserted in the same orientation, 5'-3'. GMR - $18 C 05$ and 18C05_BL2057 are the same sequences (from $D$. 
melanogaster Bloomington Stock Center Strain \#2057), but cloned in opposite directions. $18 C 05$ T7 contains the $18 C 05$ sequence of D. melanogaster T.7 strain. Comparing bristle number between $\bar{G} M R$ 18C05-GAL4 and 18C05_BL2057-GAL4 shows that the orientation of the cis-regulatory region has an effect on bristle number.

The 18C05-chimera-pBPSUw constructs were cloned using Gibson Assembly [39] by fusing together different lengths of $18 C 05$ sequences from D. yakuba Ivory Coast and D. santomea SYN2005. The different chimeras are listed in Table S9. Cloning primers were designed using NEBuilder Tools (http://nebuilder.neb.com/). Primer sequences and templates used in PCR are listed in Tables S7 and S9. To assemble the $18 C 05$ fragments in pBPSUw (Table S10), the vector was linearized by AatII and FseI restriction enzymes (New England Biolabs Inc.). After digestion thermosensitive alkaline phosphatase (FastAP, ThermoFisher Scientific) was added to the reaction to prevent self-ligation of the plasmid. PCR products and the linearized plasmid were isolated from $1 \%$ agarose gels and spin column purified. Gibson Assembly was performed as in [39], except that the assembly reactions were incubated at $37^{\circ} \mathrm{C}$ for 10 minutes and then 3 hours at $50{ }^{\circ} \mathrm{C}$ in a PCR machine. $2 \mu$ of assembly mixtures were transformed into NEB ${ }^{\circledR}$ 10-beta (New England Biolabs Inc.) competent cells and ampicillin-resistant colonies were selected on $100 \mu \mathrm{g} / \mathrm{ml}$ Amp-LB plates. The Gibson Assembly Master-mix was prepared according to [39], its components were purchased from Sigma-Aldrich.

The 18C05-yakubaSNP-pBPSUw constructs were cloned by Gibson Assembly as described above, except for 18C05yakT1008C and 18C05yakT1482C sequences, which were synthesized and cloned by GenScript $^{\circledR}$ (Table S11). The 18C05-ancestral sequences were synthesized and cloned by GenScript ${ }^{\circledR}$ into pBPSUw AatII and FseI sites, except for the 18C05_AncG869A, 18C05_AncT670G and 18C05_Anc-7SNP sequences, which were cloned by us by Gibson Assembly into pBPSUw AatII and FseI sites using the 18C05_Ancestral_Gibson_forward and 18C05_Ancestral_Gibson_reverse primers (Tables S7 and S10).

All transgenic constructs were integrated into the attP2 landing site in D. melanogaster $w^{1118}$ by BestGene Inc. The $T 1775 G$ substitution affects nucleotide position 447,055 in the Dm6 reference assembly.

\section{Genomic DNA preparations for sequencing the $18 C 05$ region}

Genomic DNA was isolated with Zymo Research Quick-DNA ${ }^{\text {TM }}$ Miniprep Plus Kit from 3 males and 3 females from the D. yakuba, D. santomea and D. teisseri lines listed in Table S12. $18 C 05$ sequences were amplified with San-Yak_lines_sequencing-For and San-Yak_lines_sequencing-Rev primers (Table S7) using Phusion ${ }^{\circledR}$ High Fidelity Polymerase (New England Biolabss).

$\underline{\text { Sequence Analysis }}$

Geneious software was used for cloning design and DNA sequence analysis. Nucleotide positions are given according to the alignment of $D$. yakuba Ivory Coast $18 C 05$ sequence with $D$. santomea SYN2005 $18 C 05$ sequence. The 18C05ancestral sequence of D. yakuba and D. santomea was reconstructed in Geneious based on the $18 C 05$ sequence alignment of Drosophila lines listed in Table S12. Manual parsimony reconstruction of all the ancestral nucleotides was unambiguous, except for one position (766, indel polymorphism), where the sequence is absent in the simulans complex and in D. santomea, while it is present in D. teissieri and polymorphic in D. yakuba. For this position we chose $D$. teissieri as the ancestral sequence. The $18 C 05$ sequences of D. melanogaster subgroup species were retrieved by BLAST from the NCBI website. Transcription Factor (TF) binding sites in 18C05 were predicted using the JASPAR CORE Insecta database (http://jaspar.genereg.net [40]). 25-60 bp sequences of $18 C 05$ were scanned with all JASPAR matrix models with 50-95\% Relative Profile Score Thresholds to test for sensitivity and selectivity [40] (Table S12). For TFs which were absent in JASPAR (Scute), we used Fly Factor Survey to analyze their putative binding affinities to the probe. As $s c$ cis-regulatory region is known to contain binding sites for Scute itself [41], we looked for Scute 
binding sites in $18 C 05,15 E 09$ and 054839 . Two putative Scute binding sites (consensus motif CAYCTGY, Fly Factor Survey [41]) were found in $15 E 09$ and 054839 but not in $18 C 05$. Given the present results, we cannot exclude the involvement of $15 E 09$ and 054839 in the evolution of hypandrial bristle evolution in D. santomea. In this paper, we decided to focus on the $18 C 05$ enhancer, whose effect could be studied in a sc mutant background.

\section{$\underline{\text { Statistical Analyses }}$}

Since bristle number is a classical type of count data, we performed statistical analysis using generalized linear models (GLM) and generalized linear mixed models (GLMM) where bristle number, the response variable, is assumed to follow a Poisson distribution [42-44]. All statistical analyses were performed using R 3.4 [45]. GLM were fitted with the function $\operatorname{glm}()$ ("stats" core package 3.5.0) and GLMM with the function glmer() ("lme4" package 1.1-14 [46] with the parameter "family" taken to be "Poisson". We tested differences in bristle number by comparing two wild-type stocks of D. yakuba with two wild-type stocks of $D$. santomea. We tested the difference between species, using a GLMM of the Poisson type (GLMM-Poisson) where the number of bristles was the response variable, species was a fixed effect to test and stock a random effect. For all other analyses, we tested differences in bristle number between genotypes using GLM of the Poisson type (GLM-Poisson) where the response variable was bristle number and genotype, a categorical variable, was the fixed effect. When we noticed important differences between residual deviance and residual degrees of freedom, we also fitted a quasi-likelihood model of the type "quasi-Poisson" (GLM-Quasi-Poisson) which allows for a model of the Poisson type, but where the variance can differ from the mean and is estimated based on a dispersion parameter (see for example [44] p. 225). For each model, in order to retain the model that fitted best to the data, analysis of deviance was performed using the anova.glm() with "test = Chisq" for GLM-Poisson and "test $=$ F" for GLM-Quasi-Poisson. When needed, we performed multiple comparisons using the glht() function and the "Holm" adjustment parameter ("multcomp" package 1.47 [47]) which performs multiple comparisons between fitted GLM parameters and yields adjusted pvalues corrected according to the Holm-Bonferroni method [48,49] also performed an exact Poisson test (R function "poisson.test") to test sample mean to a reference value assuming a Poisson distribution. Mean and 95\% confidence intervals were directly extracted from the fitted GLM and transformed using $\exp (\operatorname{coef}())$ and $\exp ($ confint.default ()$)$.

For EMSA data, response curves were compared between yak probe and san probe using an ANCOVA after natural log transformation. The unlabeled san 450x responses were compared between yak probe and san probe using a one-sided Mann-Whitney U-test.

\section{Abd-B homeodomain (Abd-B-HD) purification and EMSA}

The Abd-B-HD-pGEX-4T-1 plasmid (kindly provided by Sangyun Jeong) was transformed into BL21 (DE3) chemically competent cells. Protein expression was induced by $0.1 \mathrm{mM}$ IPTG (isopropyl- $\beta$-Dthiogalactopyranoside, Sigma Aldrich). Recombinant protein was purified from $500 \mathrm{ml}$ of bacterial culture as described in Frangioni [50] except that proteins were eluted into $50 \mathrm{mM}$ Tris- $\mathrm{HCl}, \mathrm{pH} 8.0$, $500 \mathrm{mM} \mathrm{NaCl}, 10 \mathrm{mM}$ reduced glutation (Sigma-Aldrich, G-4251) and 5\% glycerol. Concentrations and purity of the protein were determined by SDS-PAGE and Qubit 2.0 Fluorometer (Life Technologies). Protein aliquots of $20 \mu \mathrm{l}$ were snap-frozen in liquid nitrogen and stored at $-80{ }^{\circ} \mathrm{C}$.

The HPLC-purified biotinylated and non-labelled oligonucleotides (Sigma-Aldrich) were used in PCR to obtain 54 bp probes yak and san (san=yakT1775G) from $18 C 05 y a k-p B P S U w$ and 18C05yakT1775G-pBPSUw plasmid templates. Oligonucleotides are listed in Table S7. PCR products were column-purified.

We then used electrophoretic mobility shift assay (EMSA) to test whether the purified Abd-B homeodomain (ABD-B-HD) can bind directly to a 54-bp fragment of $18 C 05$ with the T1775G site at position 13 containing either $\mathrm{T}$ ( $y a k$ probe) or $\mathrm{G}$ (san probe). In each binding reaction, 20 fmol of 
probes were mixed with the purified ABD-B HD ranging from 0-1.25 $\mu \mathrm{g}(0 \mu \mathrm{g}, 0.75 \mu \mathrm{g}, 1 \mu \mathrm{g}$ and 1.25 $\mu \mathrm{g}$ ) in binding buffer containing $10 \mathrm{mM}$ TRIS pH 7.5, $50 \mathrm{mM} \mathrm{Kcl,} 0.5 \mathrm{mM}$ DTT, $6.25 \mathrm{mM} \mathrm{MgCl}_{2}, 0.05$ $\mathrm{mM}$ EDTA, $50 \mathrm{ng} / \mu \mathrm{l}$ Salmon Sperm DNA (Sigma Aldrich) and 9.00\% Ficoll 400 (Sigma Aldrich). The competition assay was performed by adding 9 pmol of unlabeled probes (450-fold excess) to the binding reaction. The reaction mixtures were incubated at $22{ }^{\circ} \mathrm{C}$ for $30 \mathrm{~min}$ and run on a nondenaturing $6 \%$ polyacrylamide gel in $0.5 \mathrm{X}$ TBE (Eurofins).

Labeling reactions were carried out with LightShift ${ }^{\mathrm{TM}}$ Chemiluminiscent EMSA Kit (ThermoFisher Scientific) according to the provider instructions with the following modifications: after electrophoresis, gels were blotted overnight in 20X SSC using the TurboBlotter Kit (GE Healthcare Life Sciences) and cross-linking of the probe to the membrane UV-light was performed at $254 \mathrm{~nm}$ and $120 \mathrm{~mJ} / \mathrm{cm} 2$ (UV stratalinker $\circledR 2400$, STRATAGENE). Chemiluminescence stained membranes were exposed to a CDD camera (FUJIFILM, LAS-4000) for 50x $10 \mathrm{sec}$ exposition time increments. The last images were used for quantification and were never saturated according to LAS 4000 software. To quantify the binding affinity of Abd-B-HD to the probes, the fractional occupancy (ratio of bound/ (free+bound) probe) was calculated for three replicate experiments (Fig. S12. E) using the intensity values of the bands measured in Image [35]. The mean fractional occupancy was significantly lower with $D$. santomea probes than with D. yakuba probes (ANCOVA, $\mathrm{F}(1,15)=10.58, \mathrm{p}=0.005$ ). We found that ABD-B-HD binds both D. yakuba and D. santomea DNA (Fig. S12. B-C). ABD-B-HD binding to the $D$. yakuba probe always resulted in a stronger shift than to the $D$. santomea probe. Furthermore, the D. santomea cold probe did not compete as efficiently as the $D$. yakuba cold probe to prevent formation of the D. yakuba DNA-ABD-B-HD complex (U-test, $\mathrm{p}=0.05$ ).

\section{Abd-B RNAi and clonal analysis}

To test whether $A b d-B$ is required for hypandrial bristle development, we reduced $A b d-B$ expression using either genetic mutations or RNAi. Two UAS.Abd-B-RNAi lines (\#51167 and \#26746) were crossed with 3 different GAL4 lines, GMR18C05-GAL4, NP5130-GAL4 and NP6333-GAL4. Crosses were kept at $29^{\circ} \mathrm{C}$ and the hypandrium phenotype was examined in 10-50 F1 males (Table S13). Using the genitalia GAL4 drivers esg-GAL4 ${ }^{N P S 130}$ [51] and NP6333 [52] to express $A b d-B . R N A i^{51167}$, we obtained 20 males out of 100 with developed hypandrium, among which two aberrant hypandrial bristle phenotypes were found, either bristle size reduction or bristle loss (Fig. S11. A-F, n=9/11 for $N P 5130, \mathrm{n}=8 / 9$ for NP6333, Table S13). Smaller bristles might arise from a delay in $s c$ expression during development [53]. Since $A b d-B$ null mutations are lethal [54], we produced mitotic mutant clones for two null mutations, $A b d-B^{M I}$ [54] and $A b d-B^{D 18}$ [55]. $A b d-B$ mutant mitotic recombinant clones were induced by the FLP/FRT system [56] using $A b d-B^{M I}$ and $A b d-B^{D 18}$ null mutations. To induce clones, ten $y w$ hsflp122; FRT82B hs-CD2 $y^{+} M(3) w^{123} / \mathrm{TM} 2$ virgin females were crossed to ten y; FRT82B Abd-B $B^{M 1}$ red[1] e[11] ro[1] ca[1]/TM6B or y; FRT82B Abd-BDI8/TM3 males (stocks were kindly provided by Ernesto Sánchez-Herrero). Crosses were flipped every 24 hours and F1 progeny were heat-shocked at $38^{\circ} \mathrm{C}$ for 1 hour at different stages of larval development: 24-48, 48-72, 72-98 and 96-120 hours after egg laying [57]. From both crosses, a total of 82 F1 males (Table S13) with the genotype of $y w$ hsflp122; FRT82B hs-CD2 $y^{+} M(3) w^{123} / F R T 82 B$ Abd-B ${ }^{M l}$ red[1] e[11] ro[1] ca[1] and $y w$ hsflp122; FRT82B hs-CD2 $y^{+} M(3) w^{123} / F R T 82 B A b d-B^{D 18}$ were examined. Hypandria were mounted and bristle clones were screened as described above. Most of the resulting males showed extreme transformation of the genitalia (Fig. S11. G-H, O-P) but 12 males out of 82 had analyzable hypandrium (twelve males for $A b d-B^{M I}$ and two males for $A b d-B^{D 18}$ ). Among them, 6 males were devoid of one or both hypandrial bristles (Table S13, Fig. S11. I-N). When hypandrial bristles were present, most of them were heterozygous for the $A b d-B$ mutation according to the visible markers associated with somatic recombination. Together, our results suggest that $A b d-B$ is required for hypandrial bristle development. 


\section{Immunostaining}

For leg disc stainings the larvae were fed on freshly prepared Formula 4-24 ${ }^{\circledR}$ Instant Drosophila Medium, Blue (Carolina) and staged by the presence of blue staining in their gut [58]. Larvae were chosen with the most clear gut, indicating a developmental stage of 1-6 hours before pupa formation [59]. Head parts of the larvae were cut and fixed in 4\% PFA in PBS pH 7.4 for 20 minutes at room temperature. For pupal leg preparations the anterior part of the pupae were cut and fixed in $4 \%$ PFA in PBS pH 7.4 for 50 minutes at room temperature. Following fixation, samples were washed three times for 5 minutes in PBS containing $0.1 \%$ Tween 20 and then permeabilized in TNT buffer (TRIS-NaCl buffer containing $0.5 \%$ Triton X-100) for 10 minutes. Samples were washed in 5\% BSA in TNT for up to 5 hours at room temperature and then incubated with rabbit anti-GFP primary antibodies (Thermofisher \#A6455) diluted in 1:1000 in TNT overnight at $4{ }^{\circ} \mathrm{C}$ and rinsed in TNT three times for 10 minutes at room temperature. Then, samples were washed in 5\% BSA in TNT for up to 5 hours at room temperature and incubated with donkey Anti-Rabbit Dylight ${ }^{\circledR} 488$ (Thermofisher) secondary antibodies diluted 1:200 in TNT overnight at $4{ }^{\circ} \mathrm{C}$. After washing the preparations in TNT for 5 minutes DNA was stained in $1 \mu \mathrm{g} / \mu \mathrm{l}$ DAPI solution (Sigma-Aldrich) for 30 minutes at room temperature. The preparations were finally washed in TNT three times for 5 minutes and the imaginal discs and pupal legs were dissected in PBS and mounted in Vectashield ${ }^{\circledR} \mathrm{H}-1000$. Images were acquired using Spinning Disc CSU-W1. Number of GFP-positive cells were counted in the z-stack using Image J [35] in a blind fashion regarding the genotypes using randomized file names. 


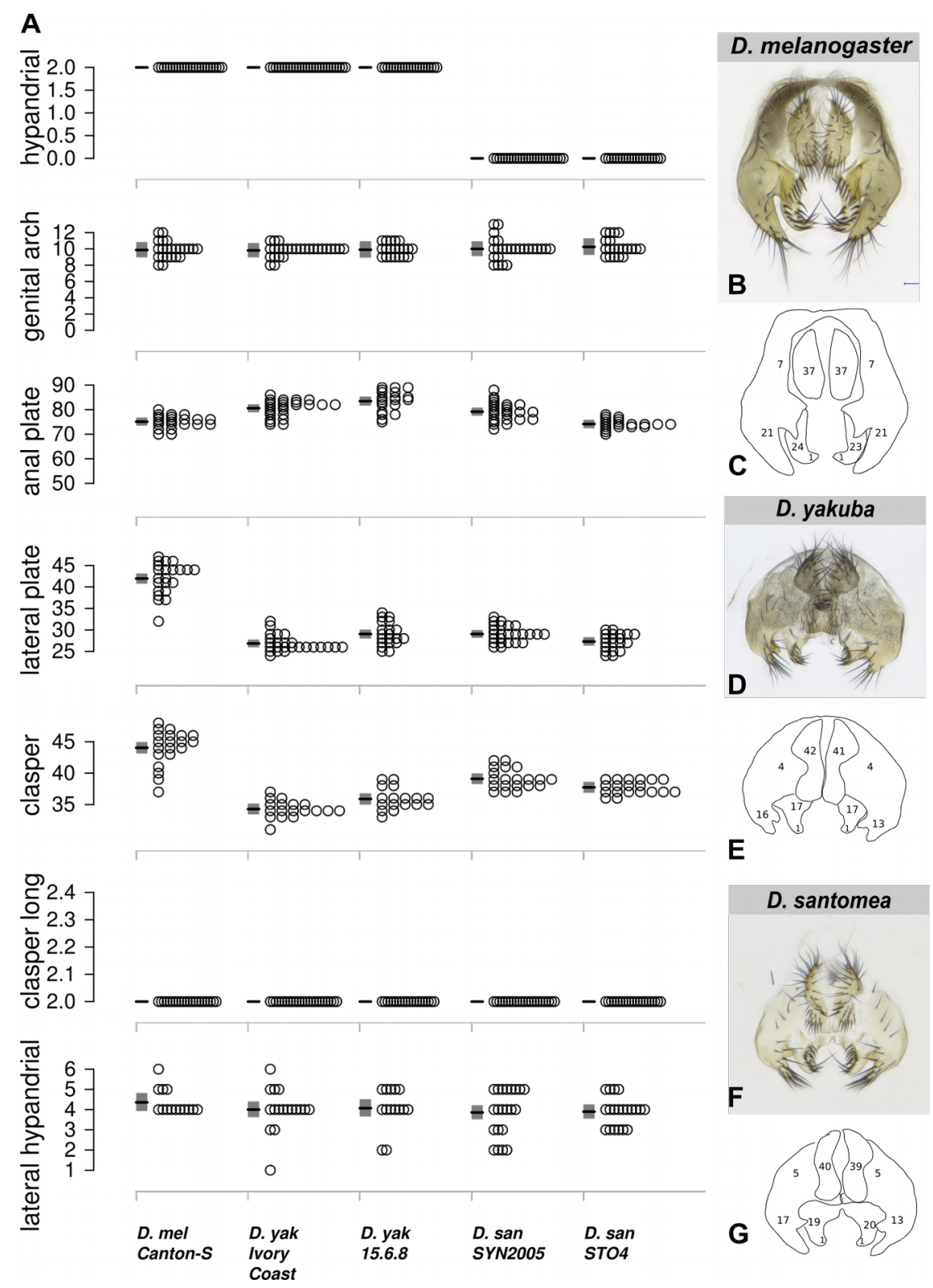

Fig. S1. Genital bristle number in D. yakuba and $D$. santomea.

(A) The bristle number of various bristle types is indicated for several strains of D. melanogaster, D. yakuba and D. santomea. Each grey dot represents one individual raised at $25^{\circ} \mathrm{C}$. Mean (black line) and 95\% confidence interval (grey rectangle) from a fitted GLM Quasi-Poisson model are shown. $D$. yakuba (yak) has fewer clasper bristles than D. santomea (san) (mean_yak $=35$, mean_san $=38$, GLMM-Poisson, Chisq $(1)=5.01, \mathrm{p}=0.025$ ) and more anal plate bristles than D. santomea (mean_yak $=82$, mean_san $=77$, GLMM-Poisson, Chisq $(1)=3.87 \mathrm{p}=0.049)$. Dissected external genitalia from $D$. melanogaster Canton-S (B), D. yakuba Ivory Coast (D) and D. santomea SYN2005 (F) and their schematic representations (C,E,G) indicating the number of bristles in each genitalia part. 


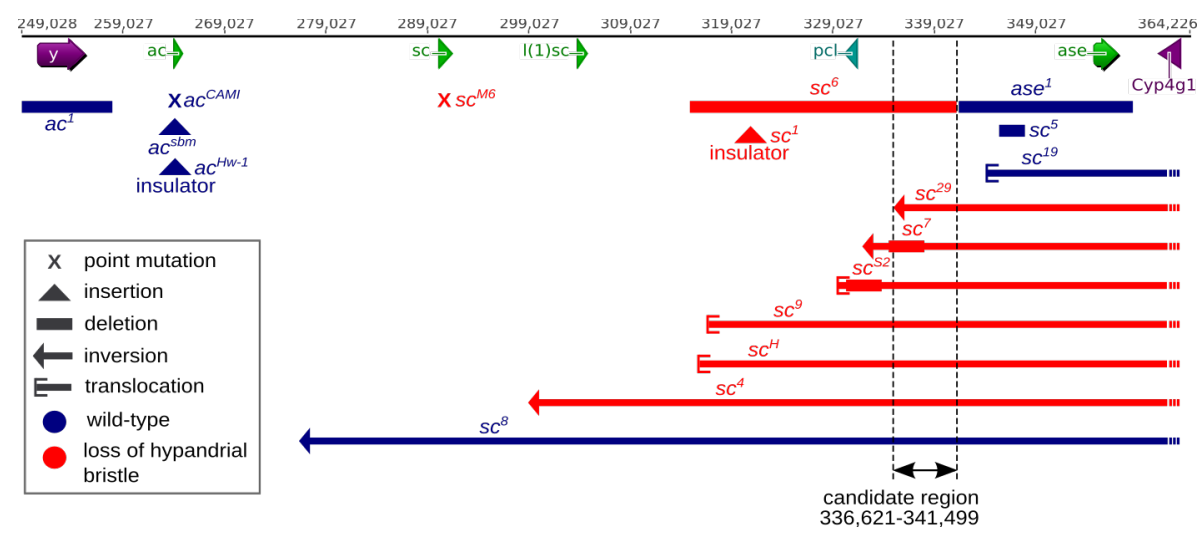

Fig. S2. Analysis of achaete-scute mutants in D. melanogaster reveals a 5-kb candidate region driving expression in hypandrial bristles.

4 Genomic organization of the $A S-C$ locus in D. melanogaster. Arrows and arrowheads indicate the coding regions of yellow $(y)$, achaete $(a)$, scute $(s c)$, lethal of scute $(l(1) s c)$, pepsinogen-like ( $p c l)$, asense (ase) and cytochrome P450-4gl (Cyp4gl) genes, respectively. The achaete-scute mutants are represented in blue if they display a wild-type phenotype of 2 bristles and in red if they show a mutant phenotype with a reduced number of hypandrial bristles. Point mutations are represented as crosses, insertions as triangles, deletions as solid horizontal bars, inversions as thin horizontal bars terminated by an arrow, and translocations as thin horizontal bars terminated by a bracket. Comparison of all the tested achaete-scute mutants suggests that a 5-kb region (black arrows) located $45 \mathrm{~kb}$ away from $s c 5^{\prime}$ coding region drives expression in the developing hypandrial bristles. 


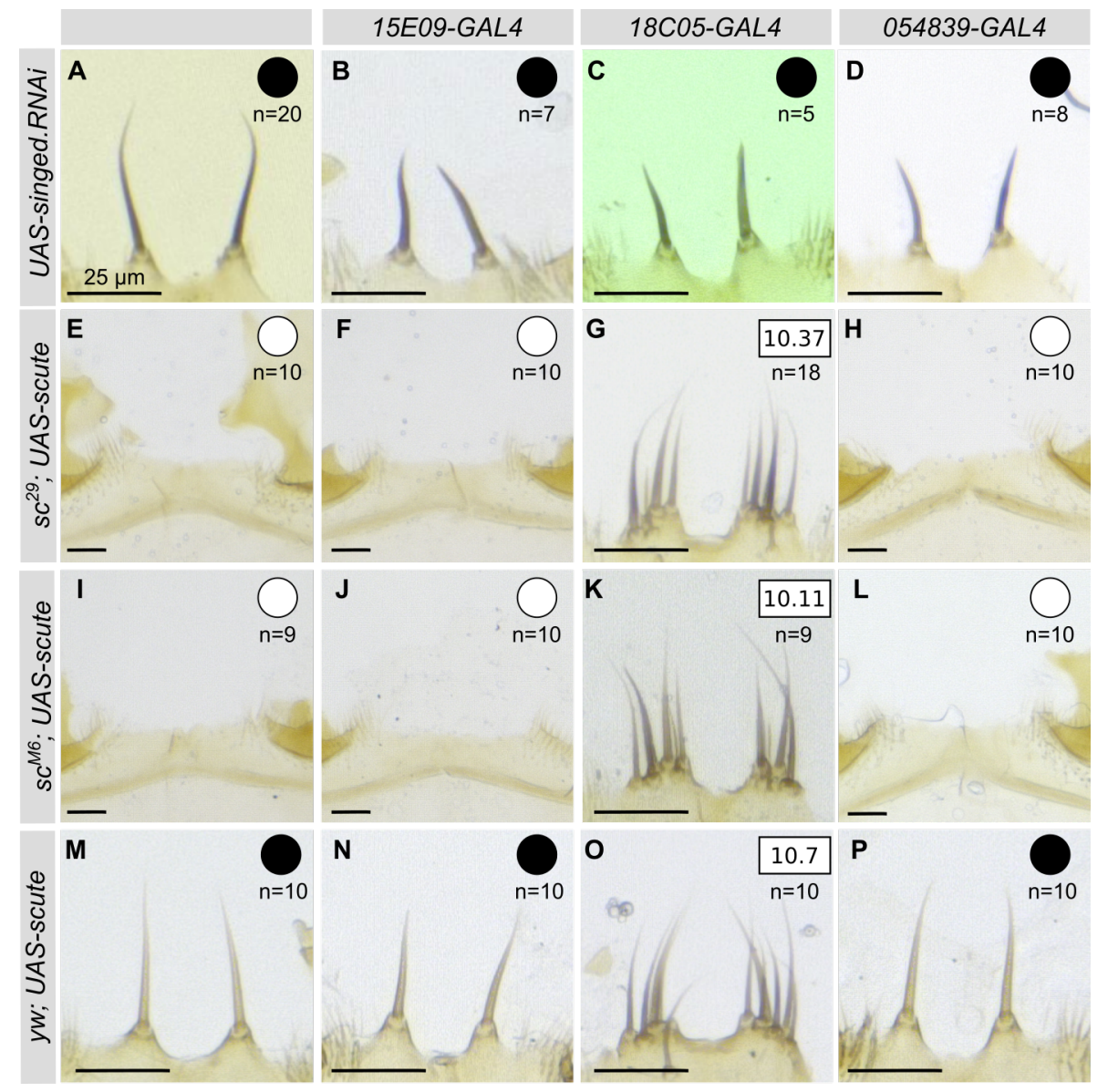

Fig. S3. Three cis-regulatory elements of the achaete-scute complex $(A S-C)$ govern expression in the hypandrium in $D$. melanogaster.

Expression of $U A S$-singed.RNAi results in singed hypandrium bristles compared to wild-type (A) with 15E09- (B), 18C05- (C) and 054839-GAL4 (D). Expression of UAS-sc (E-P) in wild-type (M-P), sc ${ }^{29}$ $(\mathrm{E}-\mathrm{H})$ or $s c^{M 6}$ (I-L) mutant background produces extra bristles with $18 C 05-G A L 4(\mathrm{G}, \mathrm{K}, \mathrm{O})$ but not in absence of a GAL4 reporter line (E,I,M) nor with 15E09- (F,J,N) and 05439-GAL4 (H,L,P). White and black circles indicate zero or two hypandrial bristles, respectively. Average bristle numbers are shown in squares $(\mathrm{G}, \mathrm{K}, \mathrm{O})$. n: number of scored individuals. (A-P) Scale bars indicate $25 \mu \mathrm{m}$. 


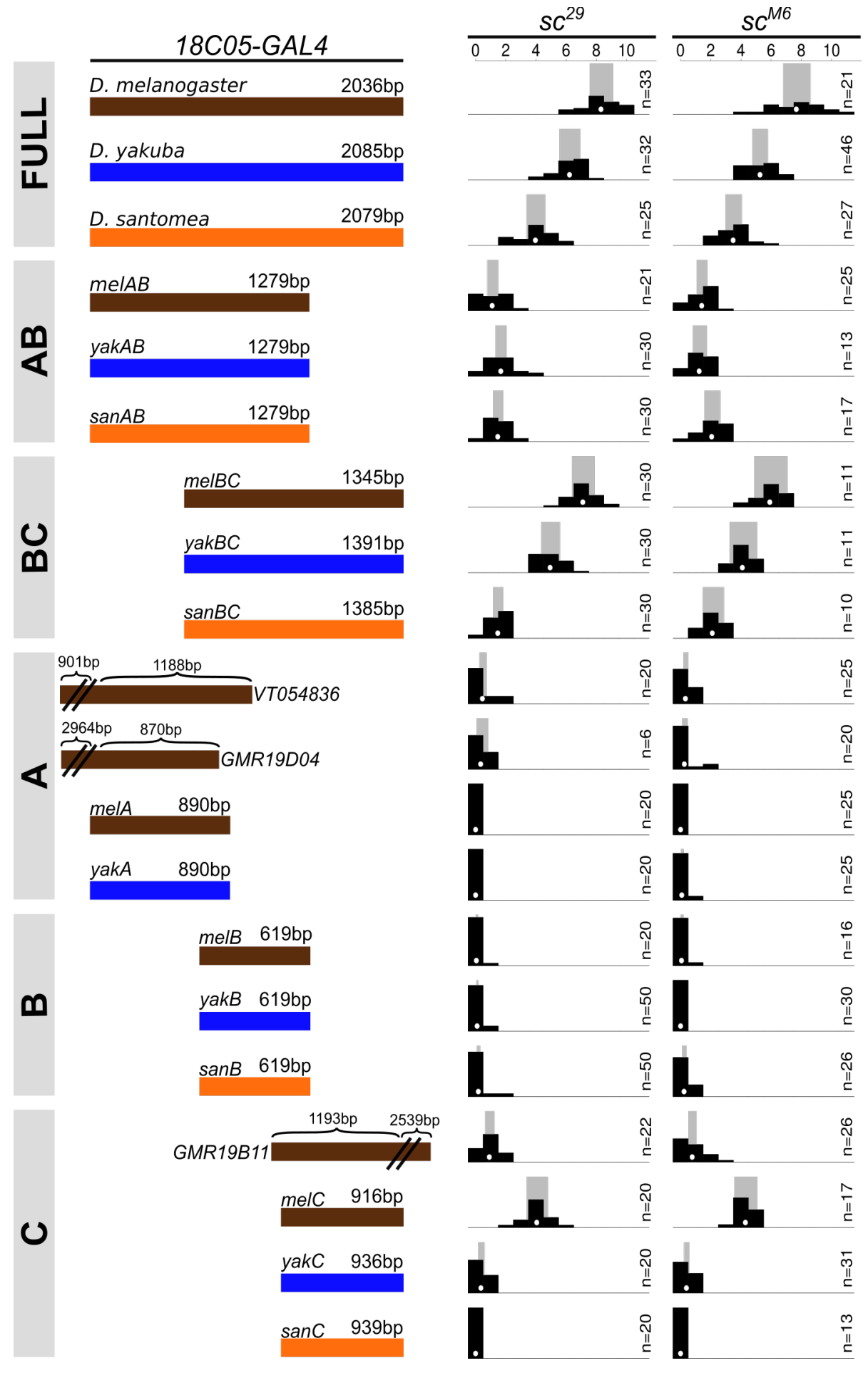
mutants by expression of GAL4 with $U A S$-sc driven by various genomic regions from $D$. melanogaster (brown), D. yakuba (blue) and D. santomea (orange). Distribution of hypandrial bristle number (black histogram), together with mean (white dot) and 95\% confidence interval (grey rectangle) from a fitted GLM Quasi-Poisson model are shown for each genotype. n: number of scored individuals. For all three species we found that smaller segments induced significantly fewer bristles than the corresponding full 
1 region $\left(\mathrm{GLM}\right.$-Quasi-Poisson, $\mathrm{F}(19,509)=161.7$, all $\mathrm{p}<0.02$ for $s c^{29} ; \mathrm{F}(19,415)=125.9, \mathrm{p}<0.03$ for the corresponding region from D. melanogaster and D. yakuba. Moreover, the $\sim 900 \mathrm{bp} 3$ ' region (region C) from the three species show striking differences: the region from $D$. melanogaster rescues about 4 bristles on average, the region from D. yakuba rescues about 0.4 bristles on average, and the region from $D$. santomea rescued no bristles. Addition of DNA 5' of the $18 C 05$ region increases hypandrial bristle numbers (VT054836 different from 0 (melA), Exact-Poisson, $\mathrm{p}<10-16$ ), whereas addition of DNA 3' of $18 \mathrm{CO} 5$ reduces the number of bristles ( GM19B11 vs. melC; GLM-QuasiPoisson, $\mathrm{F}(19,509)=161.7, \mathrm{p}<10-16$ for $s c^{29} ; \mathrm{F}(19,415)=125.9, \mathrm{p}<10-16$ for $\left.s c^{M 6}\right)$. 


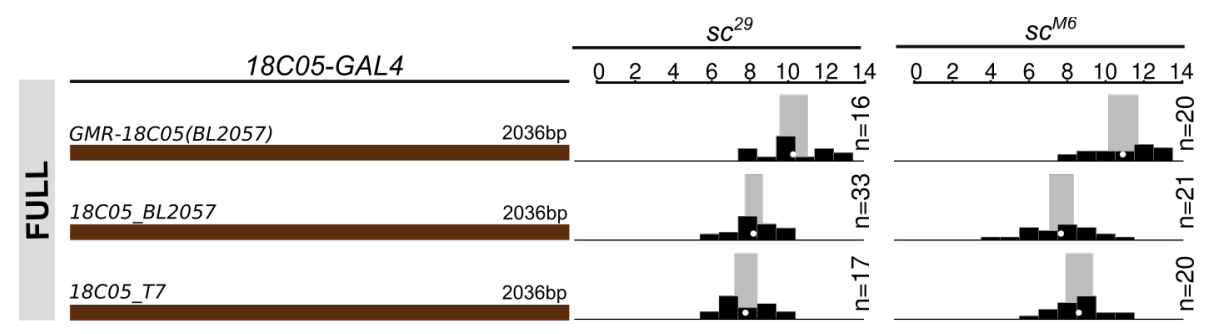

Fig. S5. Comparison of $18 C 05$ melanogaster-GAL4 constructs.

Three different $D$. melanogaster $18 C 05$ sequences were tested with $U A S$-sc in the hypandrium in $s c^{29}$ (first column) and $s c^{M 6}$ (second column). Distribution of bristle number (black histogram), together with mean (white dot) and 95\% confidence interval (grey rectangle) from a fitted GLM Quasi-Poisson model are shown. Hypandrial bristle number, for GMR-18C05 (BL2057) is significantly higher than 18C05_BL2057 and 18C05_T7 in both backgrounds (GLM-Quasi-Poisson, F(2, 63) = 16.88 , both $\mathrm{p}<$ $10^{\wedge}-6$ for $s c^{29} ; \mathrm{F}(2,58)=20.9, \mathrm{p}<10^{\wedge}-10$ and $\mathrm{p}<10^{\wedge}-5$ for $\left.s c^{M 6}\right)$. GMR-18C05 (BL2057) comes from the Janelia Farm collection (35). 18C05_BL2057 and 18C05_T7 were cloned in this study. GMR$18 C 05$ and 18C05_BL2057 are the same sequence (from D. melanogaster Bloomington Stock Center Strain \#2057), cloned in opposite direction. 18C05_T7 contains the 18C05 sequence of $D$.

melanogaster T.7 strain. The GMR-18C05 fragment is inserted in the expression vector 3'-5' compared to the D. melanogaster genome sequence. In contrast, the 18C05_BL2057 and 18C05_T7 are cloned 5'3'. Comparing bristle number between GMR-18C05 and 18C05_BL2057 shows that the orientation of the cis-regulatory region has an effect on bristle number. All our $18 C 05$ constructs were inserted in the same orientation, 5'-3'. 
bioRxiv preprint doi: https://doi.org/10.1101/313197; this version posted July 16, 2018. The copyright holder for this preprint (which was not certified by peer review) is the author/funder. All rights reserved. No reuse allowed without permission.

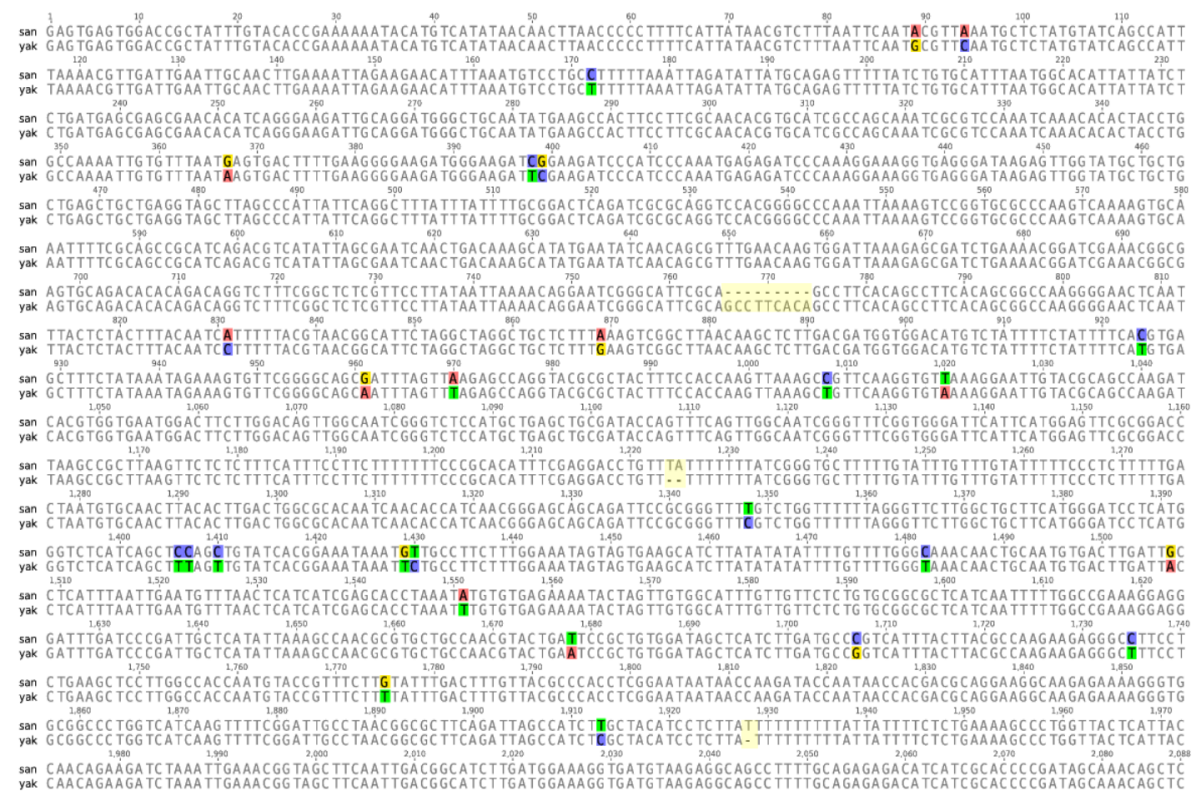

Fig. S6. Alignment of $18 C 05$ sequence from D. santomea SYN2005 (san) and D. yakuba Ivory 3 Coast (yak). 


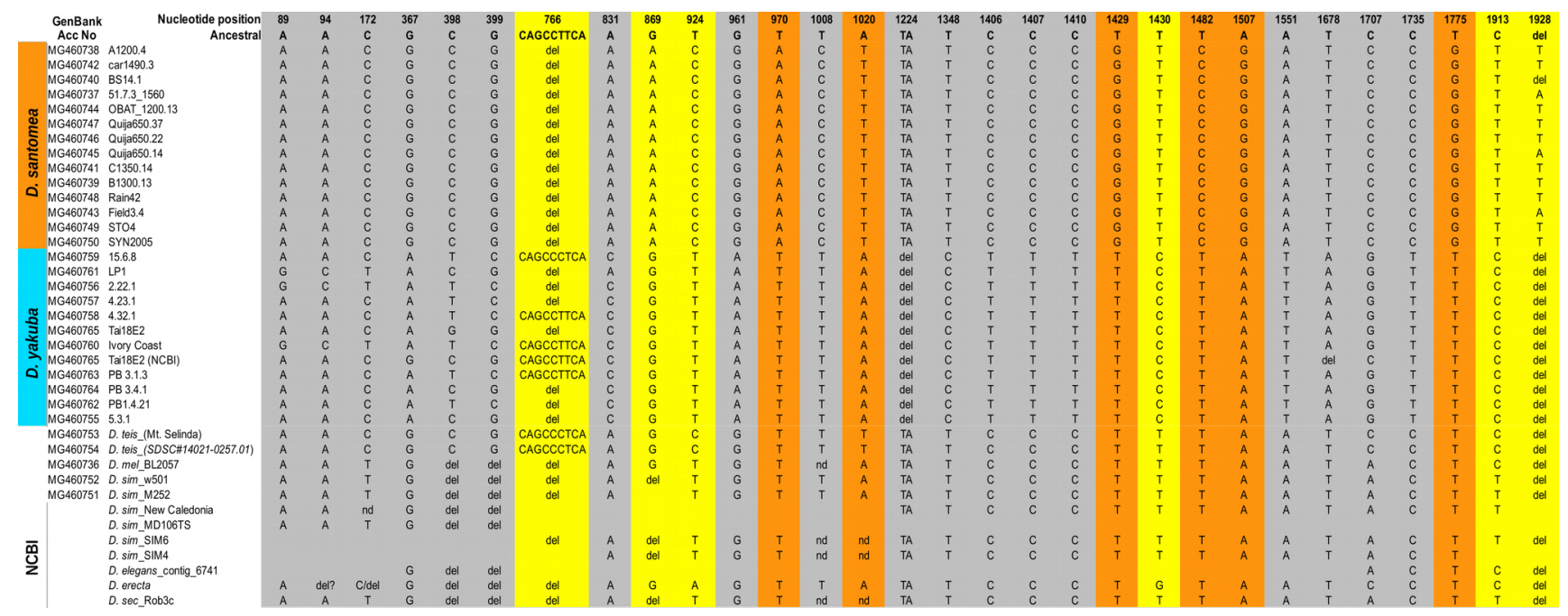

Fig. S7. Twelve substitutions are fixed in D. santomea $18 C 05$.

Summary of the alignment of $18 C 05$ sequences from different lines of 7 species. Only divergent sites within and between D. yakuba and D. santomea are shown (divergence to D. teissieri is not shown). Nucleotide positions are indicated in the first row. The $D$. santomea-specific substitutions are labelled in orange when they are fixed in the other D. melanogaster subgroup species and in yellow when they are variable in at least one of the other $D$. melanogaster subgroup species. The reconstructed ancestral sequence of $D$. santomea and D. yakuba is shown at the top. Del: deletion. NCBI: sequences were retrieved from NCBI BLAST. GenBank accession numbers are shown in the first column for the sequences obtained in this study. 


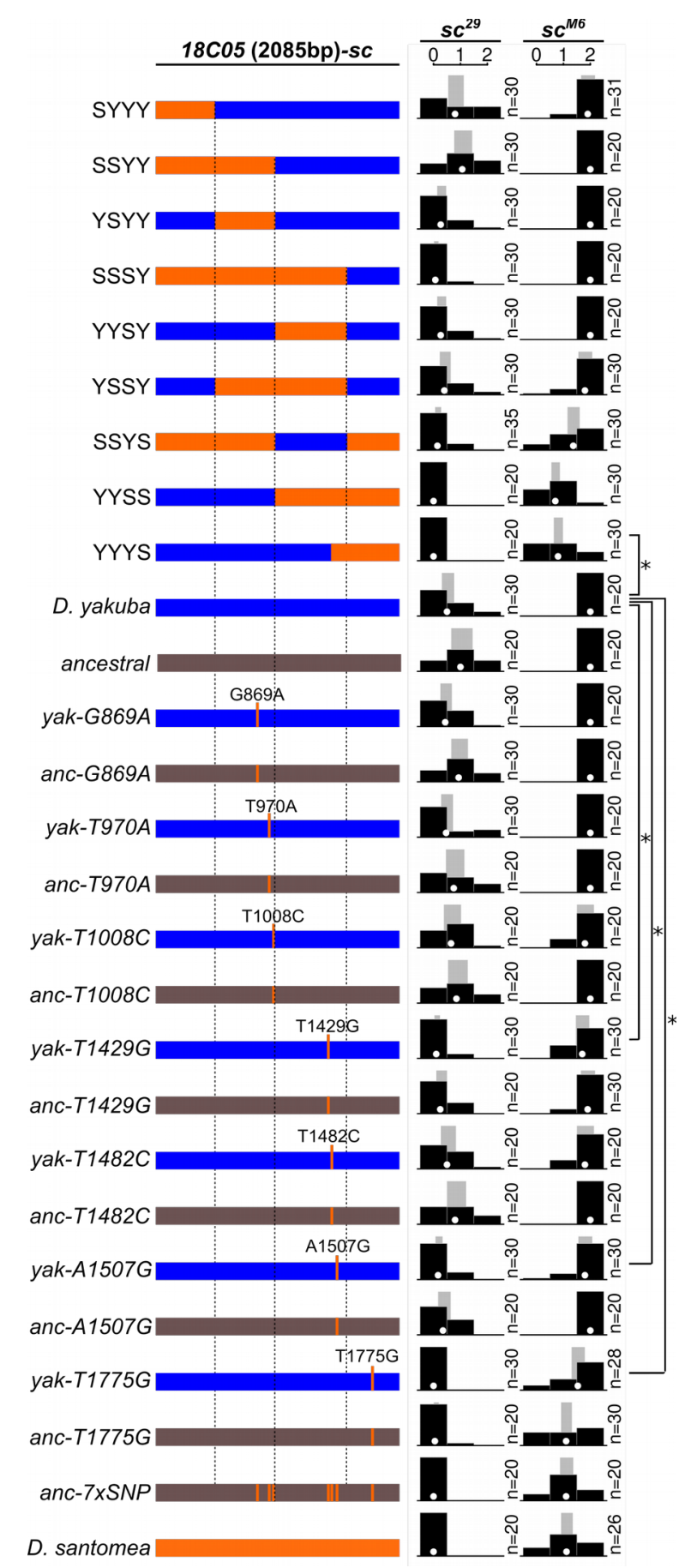
tested for their ability to rescue hypandrial bristles in $s c^{29}$ (left column) and $s c^{M 6}$ (right column) mutant backgrounds. The $18 C 05$ sequence from $D$. yakuba (blue, labeled with Y) and from $D$. santomea (orange, labeled with $\mathrm{S}$ ) were divided into four subregions which were then fused together in different chimeric combinations. D. santomea-specific substitutions are represented as dark orange bars. Seven D. santomea-specific substitutions were introduced into either the D. yakuba region (blue) or the ancestrally reconstructed $18 C 05$ region (dark grey). n: number of scored individuals. *: $\mathrm{p}<0.05$. Replacing the $5^{\prime}$ half (1-1020 bp) of D. yakuba $18 C 05$ by D. santomea corresponding region has little 
1 or no effect (GLM-Quasi-Poisson, $\mathrm{F}(26,658)=12.09$, SSYY versus D. yakuba: $\mathrm{p}=0.031$ in $s c^{29}$, YSYY not different from D. yakuba: $\mathrm{p}=0.432$ in $s c^{29}$, SYYY not different from D. yakuba: $\mathrm{p}=0.432$ in $s c^{29}$ ) whereas replacing the 3' end (1573-2088 bp) decreases bristle number (GLM-Quasi-Poisson, F(26,618) $=16.04$, YYYS versus D. yakuba: $\mathrm{p}<10-16$ in $s c^{M 6}$, YYSS versus D. yakuba: $\mathrm{p}<10-16$ in $\left.s c^{M 6}\right)$. The 3' middle part (1021-1572 bp) has no effect (YYSY not different from D. yakuba: $\mathrm{p}=0.432$ in $s c^{29}$, YYSS versus YYYS: $\mathrm{p}=0.579$ in $\left.s c^{M \sigma}\right)$ except in presence of the $D$. santomea $5^{\prime}$ half $(1-1020 \mathrm{bp})$, in which case it decreases bristle number (SSSY versus SSYY: $\mathrm{p}<10-4$ in $s c^{29}$ ). Our analysis of chimeric constructs thus indicates that at least two changes, in regions 1021-1572 and 1573-2088, contribute to the reduced ability of $D$. santomea $18 C 05$ to produce hypandrial bristles. Three substitutions significantly decreased the number of rescued bristles in the $D$. yakuba and/or the ancestral sequence (T1429G: $\mathrm{p}=0.021$ and $\mathrm{p}=0.009$ in $s c^{29}$, respectively; $\mathrm{A} 1507 \mathrm{G}: \mathrm{p}=0.066$ and $\mathrm{p}=0.03$ in $s c^{29}$, respectively; T1775G: $\mathrm{p}=0.013$ and $\mathrm{p}=10-8$ in $s c^{M 6}$, respectively). 


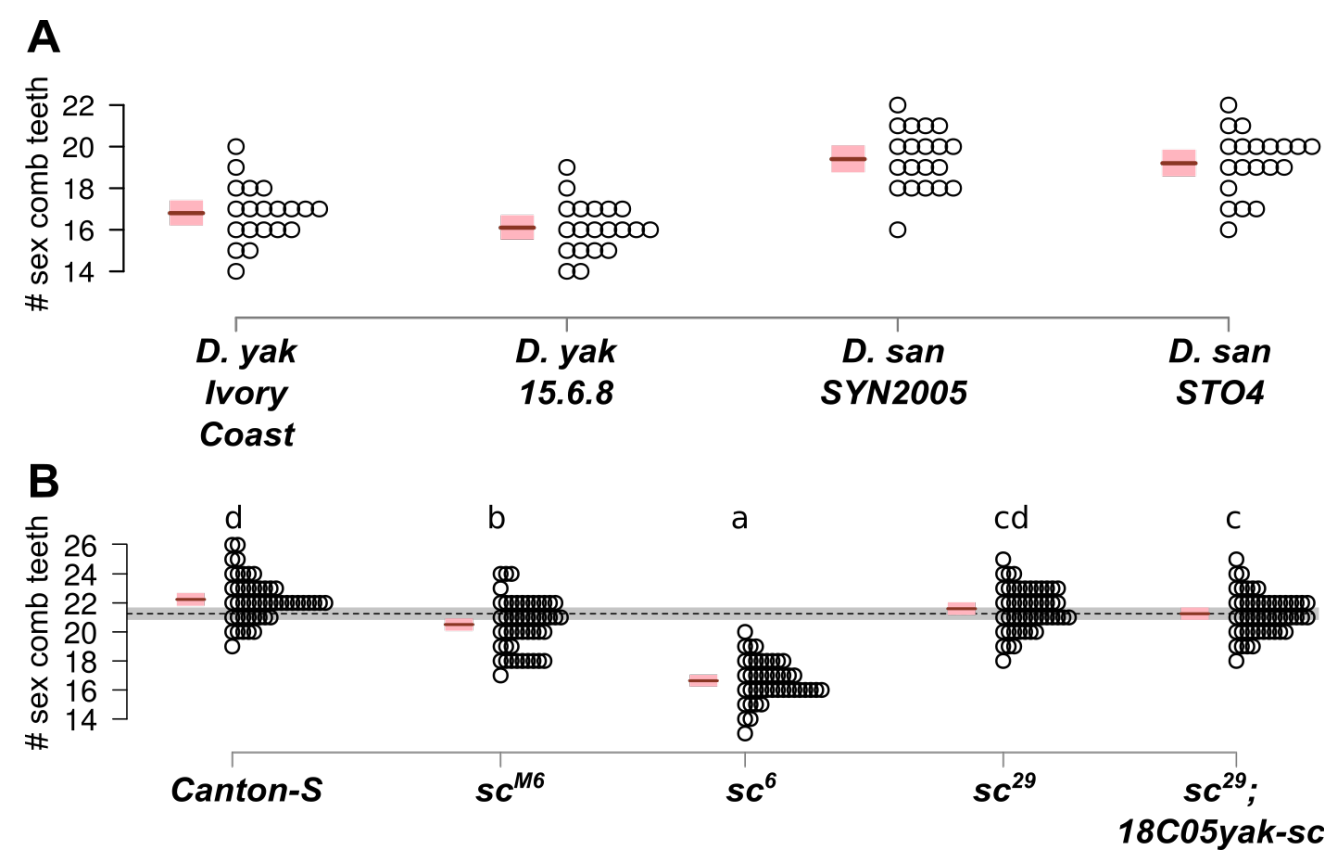

Fig. S9. Sex comb tooth number in D. yakuba and D. santomea and D. melanogaster scute mutants.

Each circle represents one individual raised at $25^{\circ} \mathrm{C}$. Mean (brown line) and $95 \%$ confidence interval (pink rectangle) from a fitted GLM Quasi-Poisson model are shown. (A) Sex comb tooth number is significantly different between $D$. yakuba and D. santomea (GLM-Poisson, Chisq $(1)=2.76, \mathrm{p}=0.007$ ). (B) $s c^{M 6}$ and $s c^{6}$ have significantly different sex comb tooth number than Canton-S. Letters indicate the results of all-pairwise comparisons after Holm-Bonferroni correction. Two genotypes are significantly different from each other $(\mathrm{p}<0.05)$ when they do not share a letter. Sex comb tooth number of $s c^{29}$ mutant males is not significantly different from wild-type $(\mathrm{F}(4,239)=100.06, \mathrm{p}=0.09)$ and the $18 C 05 y a k-s c$ construct does not significantly increase sex comb tooth number in the $s c^{29}$ background $\left(s c^{29}\right.$ versus $\left.s c^{29} 18 C 05 y a k-s c, \mathrm{~F}(4,239)=100.06, \mathrm{p}=0.27\right)$, so we did not examine the effects of $D$. santomea substitutions in the $s c^{29}$ background. 


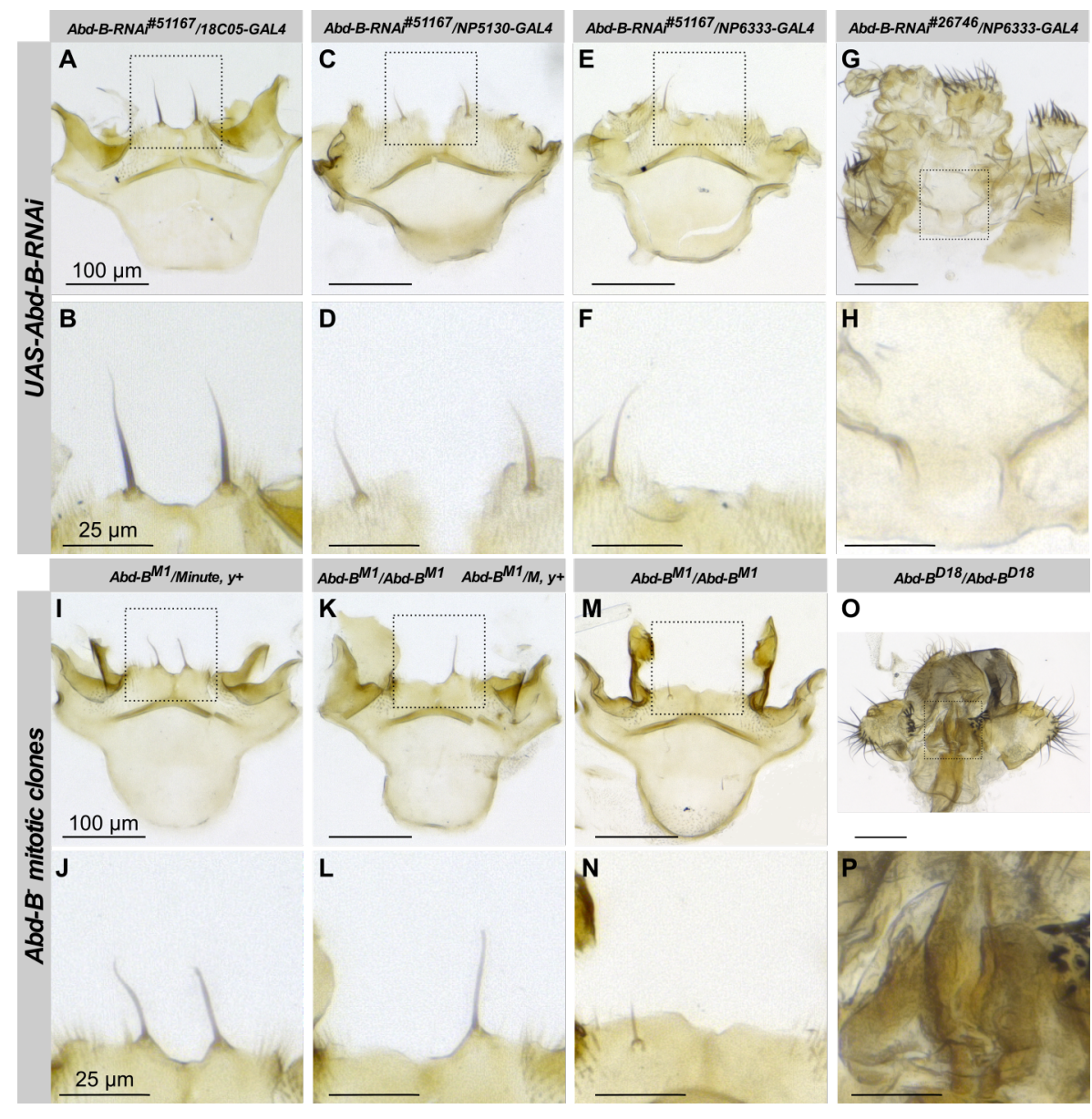

Fig. S10. Hypandrial bristle development affected in $A b d-B-R N A i$ and $A b d-B^{-}$clones.

Wild-type hypandrial bristles. (B) In $A b d-B-R N A i^{\# 51167} / G M R 18 C 05-G A L 4$ males the hypandrial bristles are thiner. (C) In $A b d-B-R N A i^{\# 51167} / N P 5130-G A L 4$ hypandrial bristles are thiner and shorter. (D) In $A b d-B-R N A i^{\# 51167} / N P 6333-G A L 4$ hypandrial bristles are thiner or lost. (I-N) In $A b d-B^{-}$clones hypandrial bristles are lost. Clones were selected in Minute, $y^{+}$background. One hypandrium with two Minute, $y^{+}$ bristles (I-J), one hypandrium with only Minute, $y^{+}$bristle (K-L) and one hypandrium with no hypandrial bristle $(\mathrm{M}-\mathrm{N})$ are shown. Extreme transformations of genitalia with abnormal hypandrium are shown in $A b d-B-R N A i^{\# 26746} / N P 6333-G A L 4(\mathrm{G}-\mathrm{H})$. 


\section{A}

Abd-B

yak GTACCGTTTCTITPATITGACTTTGTTACGCCCACCTCGGAATAATAACCAAGA

san = yakT1775G GTACCGTTTCTIGTATTIGACTTTGTTACGCCCACCTCGGAATAATAACCAAGA
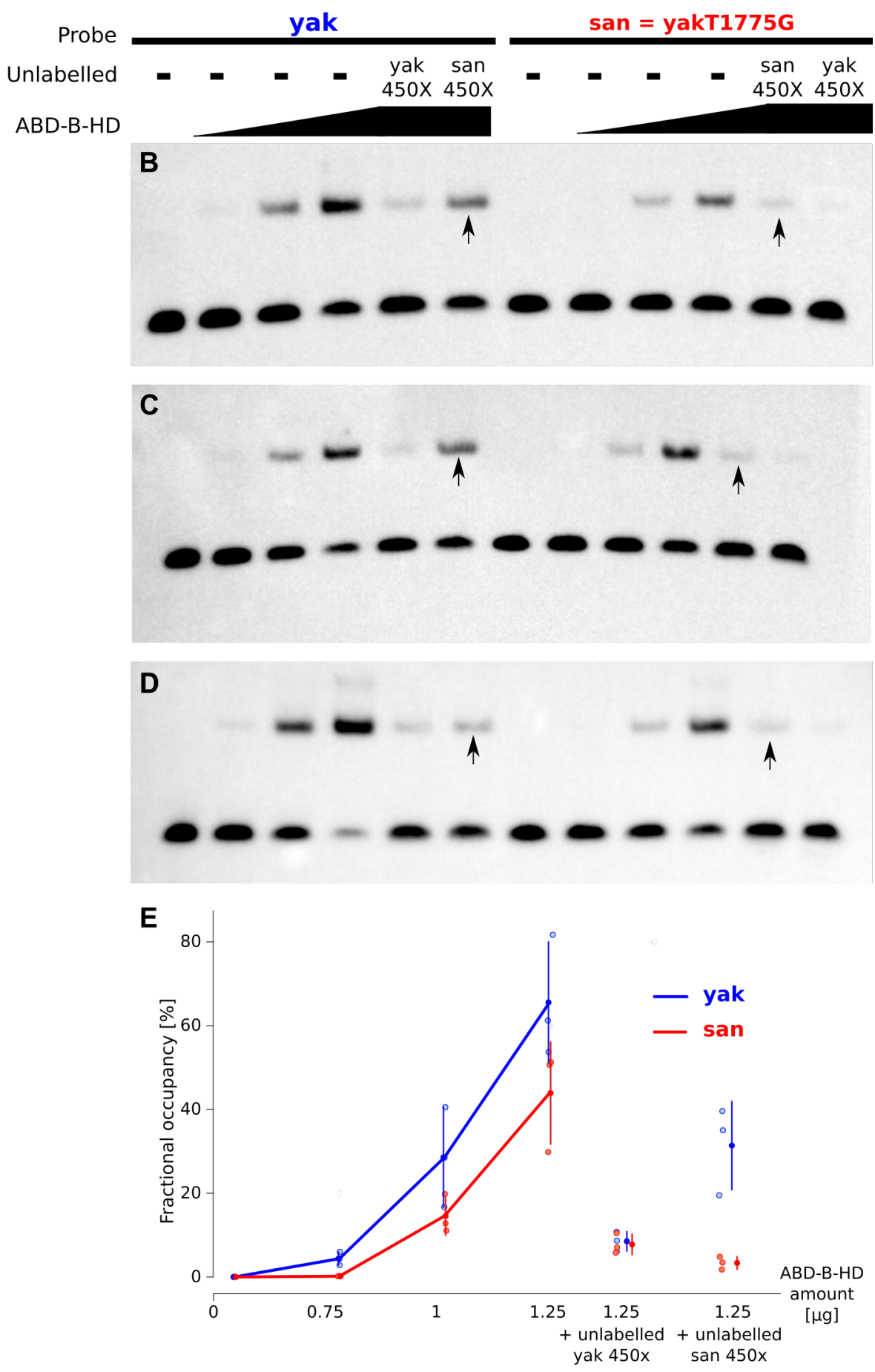

Fig. S11. D. santomea-specific substitution T1775G affects Abd-B binding.

(A) Two double-stranded oligonucleotides were used in EMSA, one with D. yakuba sequence (yak, blue) and one with $D$. santomea sequence (san=yakT1775G, red). The putative Abd-B binding site is shown in black. (B-D) Threee paralel EMSA were performed with increasing amount of Abd-B-HD $(0.75 \mu \mathrm{g}, 1.0 \mu \mathrm{g}$ and $1.25 \mu \mathrm{g})$ and yak probe or san probe. In competitor lanes $450 \mathrm{x}$ excess of 
$1 \quad$ unlabelled yak probe or san probe were added to the binding reaction. In all experiments san probe binds less than yak probe to Abd-B-HD, and unlabelled san probe competes less the band shift of the labelled probe compared to the yak probe (indicated with arrows). (E) Quantification of EMSA shifts. Fractional occupancy (ratio of bound/(free+bound) probe) is shown for three parallel experiments. Mean (dot) and standard deviation (bar) are shown for yak and san probes, in blue and red, 6 respectively. 
Table S1. Fly lines used.

See Table S4 for GAL4 lines others than DC-GAL4 and Table S5 for achaete-scute mutant lines.

\begin{tabular}{|c|c|c|}
\hline Species Name & Strain Name & Origin \\
\hline D. melanogaster & 3870 & $\begin{array}{l}\text { Given by T. Long. } \\
\text { RVC 3. Collected from Riverside, California, USA in } 1963\end{array}$ \\
\hline D. melanogaster & 3844 & $\begin{array}{l}\text { Given by T. Long. San Diego Stock Center \#14021-0231.60 } \\
\text { BS1. Collected from Barcelona, Spain in } 1954\end{array}$ \\
\hline D. melanogaster & 3841 & $\begin{array}{l}\text { Given by T. Long. San Diego Stock Center \#14021-0231.59 } \\
\text { BOG1. Collected from Bogota, Colombia in } 1962\end{array}$ \\
\hline D. melanogaster & 3852 & $\begin{array}{l}\text { Given by T. Long. San Diego Stock Center \#14021-0231.64 } \\
\text { KSA2. Collected in } 1963\end{array}$ \\
\hline D. melanogaster & 3864 & $\begin{array}{l}\text { Given by T. Long. San Diego Stock Center \# 14021-0231.68 } \\
\text { KI2. Collected from Israel in } 1954\end{array}$ \\
\hline D. melanogaster & T.7 & $\begin{array}{l}\text { Given by T. Long. San Diego Stock Center \#14021-0231.07 } \\
\text { Collected from Taiwan in } 1968\end{array}$ \\
\hline D. melanogaster & T.4 & $\begin{array}{l}\text { Given by T. Long. San Diego Stock Center \#14021-0231.04 } \\
\text { Collected from Kuala Lumpur, Malaysia in } 1962\end{array}$ \\
\hline D. melanogaster & 3875 & $\begin{array}{l}\text { Given by T. Long. San Diego Stock Center \#14021-0231.69 } \\
\text { VAG1. Collected from Athens, Greece in 1965 }\end{array}$ \\
\hline D. melanogaster & 3886 & $\begin{array}{l}\text { Given by T. Long. Wild 5B. Collected from Red Top } \\
\text { Mountain, Georgia in } 1966\end{array}$ \\
\hline D. melanogaster & T.1 & $\begin{array}{l}\text { Given by T. Long. San Diego Stock Center \#14021-0231.04 } \\
\text { Collected from Ica, Peru in } 1956\end{array}$ \\
\hline D. melanogaster & 3839 & $\begin{array}{l}\text { Given by T. Long. San Diego Stock Center \# 14021-0231.58 } \\
\text { BER1. Collected from Bermudas in } 1954 .\end{array}$ \\
\hline D. melanogaster & 3846 & $\begin{array}{l}\text { Given by T. Long. San Diego Stock Center \#14021-0231.62 } \\
\text { CA1. Collected from Cape Town, South Africa. }\end{array}$ \\
\hline D. melanogaster & Sam & $\begin{array}{l}\text { Given by T. Long. DSPR line. originally from TFC Mackay } \\
\text { Sam; ry506 }\end{array}$ \\
\hline D. melanogaster & iso-1 & $\begin{array}{l}\text { Bloomington Stock Center \#2057 (ordered) } \\
\mathrm{y}[1] ; \text { Gr22b[iso-1] Gr22d[iso-1] cn[1] CG33964[iso-1] bw[1] } \\
\text { sp[1]; LysC[iso-1] MstProx[iso-1] GstD5[iso-1] Rh6[1] }\end{array}$ \\
\hline D. melanogaster & Canton-S & Kyoto DGGR \#105666 (Given by Roger Karess) \\
\hline D. melanogaster & $\begin{array}{l}\operatorname{dor}[4] / C(1) R M, y[1] \\
w[1] f[1]\end{array}$ & Bloomington Stock Center \#35 (ordered) \\
\hline D. melanogaster & $\begin{array}{l}\text { Nup98- } \\
96[339] / T M 3, S b[1]\end{array}$ & Bloomington Stock Center \#4951 (ordered) \\
\hline D. melanogaster & $D f(3 R) D 605 / T M 3$ & Bloomington Stock Center \#823 (ordered) \\
\hline
\end{tabular}




\begin{tabular}{|c|c|c|}
\hline & $\operatorname{Sb}[1] \operatorname{Ser}[1]$ & \\
\hline D. melanogaster & DC002 & $\begin{array}{l}\text { Bloomington Stock Center \#30213 (ordered) } \\
\text { w1118; Dp(1;3)DC002, PBac }\{\text { DC002\}VK00033 }\end{array}$ \\
\hline D. melanogaster & DC003 & $\begin{array}{l}\text { Bloomington Stock Center \#30214 (ordered) } \\
\text { w1118; Dp(1;3)DC003, PBac }\{\text { DC003\}VK00033 }\end{array}$ \\
\hline D. melanogaster & DC004 & $\begin{array}{l}\text { Bloomington Stock Center \#30215 (ordered) } \\
\text { w1118; Dp(1;3)DC004, PBac }\{\text { DC004\}VK00033/TM6C, Sb1 }\end{array}$ \\
\hline D. melanogaster & DC006 & $\begin{array}{l}\text { Bloomington Stock Center \#30217 (ordered) } \\
\text { w1118; Dp(1;3)DC006, PBac }\{\text { DC006\}VK00033/TM6C, Sb1 }\end{array}$ \\
\hline D. melanogaster & DC097 & $\begin{array}{l}\text { Bloomington Stock Center \#31440 (ordered) } \\
\text { w1118; Dp(1;3)DC097, PBac }\{\text { DC097\} VK00033/TM6C, Sb1 }\end{array}$ \\
\hline D. melanogaster & DC098 & $\begin{array}{l}\text { Bloomington Stock Center \#31441 (ordered) } \\
\text { w1118; Dp(1;3)DC098, PBac }\{\text { DC098\}VK00033 }\end{array}$ \\
\hline D. melanogaster & DC007 & $\begin{array}{l}\text { Bloomington Stock Center \#30218 (ordered) } \\
\text { w1118; Dp(1;3)DC007, PBac }\{\text { DC007\} VK00033/TM6C, Sb1 }\end{array}$ \\
\hline D. melanogaster & DC008 & $\begin{array}{l}\text { Bloomington Stock Center \#30745 (ordered) } \\
\text { w1118; Dp(1;3)DC008, PBac }\{\text { DC008\}VK00033 }\end{array}$ \\
\hline D. melanogaster & DC009 & $\begin{array}{l}\text { Bloomington Stock Center \#30219 } \\
\text { w1118; Dp(1;3)DC009, PBac }\{\text { DC009\} VK00033 }\end{array}$ \\
\hline D. melanogaster & DC012 & $\begin{array}{l}\text { Bloomington Stock Center \#30222 (ordered) } \\
\text { w1118; Dp(1;3)DC012, PBac }\{\text { DC012\}VK00033 }\end{array}$ \\
\hline D. melanogaster & DC099 & $\begin{array}{l}\text { Bloomington Stock Center \#30749 (ordered) } \\
\text { w1118; Dp(1;3)DC099, PBac\{DC099\}VK00033 }\end{array}$ \\
\hline D. melanogaster & DC013 & $\begin{array}{l}\text { Bloomington Stock Center \#30746 (ordered) } \\
\text { w1118; Dp(1;3)DC013, PBac\{DC013\}VK00033 }\end{array}$ \\
\hline D. melanogaster & DC014 & $\begin{array}{l}\text { Bloomington Stock Center \#31434 (ordered) } \\
\text { w1118; Dp(1;3)DC014, PBac }\{\text { DC014\}VK00033 }\end{array}$ \\
\hline D. melanogaster & DC400 & $\begin{array}{l}\text { Bloomington Stock Center \#30795 (ordered) } \\
\text { w1118; Dp(1;3)DC400, PBac }\{\text { DC400\}VK00033 }\end{array}$ \\
\hline D. melanogaster & DC019 & $\begin{array}{l}\text { Bloomington Stock Center \#30223 (ordered) } \\
\text { w1118; Dp(1;3)DC019, PBac }\{\text { DC019\}VK00033 }\end{array}$ \\
\hline D. melanogaster & DC436 & $\begin{array}{l}\text { Bloomington Stock Center \#33487 (ordered) } \\
\text { w1118; Dp(1;3)DC436, PBac }\{\text { DC436\} VK00033/TM6C, Sb1 }\end{array}$ \\
\hline D. melanogaster & DC401 & $\begin{array}{l}\text { Bloomington Stock Center \#30796 (ordered) } \\
\text { w1118; Dp(1;3)DC401, PBac }\{\text { DC401\} VK00033 }\end{array}$ \\
\hline D. melanogaster & $D C-G A L 4$ & $\begin{array}{l}y w ; D C-G A L 4, U A S-G F P / T M 6 B \\
\text { Given by V. Stamataki (Pat Simpson lab). }\end{array}$ \\
\hline D. melanogaster & $U A S$-forked.RNAi ${ }^{33200}$ & VDRC \#33200 (ordered) \\
\hline D. melanogaster & $\begin{array}{l}U A S- \\
\text { singed.RNAi } i^{105747}\end{array}$ & VDRC \#105747 (ordered) \\
\hline
\end{tabular}




\begin{tabular}{|c|c|c|}
\hline D. melanogaster & $U A S$-forked.RNAi $i^{24632}$ & VDRC \#24632 (ordered) \\
\hline D. melanogaster & $U A S-a c . R N A i^{100647}$ & VDRC \#100647 (ordered) \\
\hline D. melanogaster & $\begin{array}{l}U A S- \\
\text { singed.RNAi } i^{32579}\end{array}$ & VDRC \#32579 (ordered) \\
\hline D. melanogaster & $\begin{array}{l}\text { UAS- } \\
\text { forked.RNAi }{ }^{103813}\end{array}$ & VDRC \#103813 (ordered) \\
\hline D. melanogaster & $U A S-S c . R N A i^{105951}$ & VDRC \#105951 (ordered) \\
\hline D. melanogaster & $y w ; U A S-y$ & $\begin{array}{l}\text { Bloomington Stock Center \#3043 } \\
\mathrm{y}[1] \mathrm{w}[1118] ; \mathrm{P}\{\mathrm{w}[+\mathrm{mC}]=\mathrm{UAS}-\mathrm{y} . \mathrm{C}\} \mathrm{MC} 1\end{array}$ \\
\hline D. melanogaster & $\begin{array}{l}y w ; U A S-y T M 3 / p n r- \\
G A L 4\end{array}$ & $\begin{array}{l}\text { Given by M. Rebeiz. } \\
\text { y w; UAS-y TM3 Ser / pnr-GAL4 }\end{array}$ \\
\hline D. melanogaster & $U A S-m C D 8-G F P$ & Given by V. Brodu. GFP transgene on second chromosome \\
\hline D. melanogaster & $\begin{array}{l}\text { w,UAS-Dcr2 } \\
\text { Pin/CyO }\end{array}$ & Bloomington Stock Center \#24644 (ordered) \\
\hline D. melanogaster & UAS-scute & Bloomington Stock Center \#51672 (ordered) \\
\hline D. melanogaster & $G F P-s c$ & $\begin{array}{l}\text { GFP inserted at the scute locus by CRISPR-mediated } \\
\text { homologous recombination, which produces Scute protein } \\
\text { with GFP sequence fused at the N terminus (Given by F. } \\
\text { Schweisguth) [59b] }\end{array}$ \\
\hline D. melanogaster & $\begin{array}{l}y w ; U A S-A b d- \\
B . R N A i^{51167}\end{array}$ & Bloomington Stock Center \#51167 (ordered) \\
\hline D. melanogaster & $\begin{array}{l}y w ; U A S-A b d- \\
B . R N A i^{26746}\end{array}$ & Bloomington Stock Center \#26746 (ordered) \\
\hline D. melanogaster & $y w ; N P 5130-G A L 4$ & Kyoto Drosophila Stock Center (ordered) \\
\hline D. melanogaster & $y w ; N P 6333-G A L 4$ & Kyoto Drosophila Stock Center (ordered) \\
\hline D. simulans & & Collected from Marrakech, Morocco by J. David in 2010 \\
\hline D. mauritiana & & Collected from Mauritius Island in 1985 \\
\hline D. sechellia & GFP & $\begin{array}{l}\text { San Diego Stock Center \#14021-0248.32 } \\
\text { w[1] ; pBac(3xP3-EGFPafm)::MCS::(pW8 mini-white) }\end{array}$ \\
\hline D. yakuba & Ivory Coast & $\begin{array}{l}\text { Given by D. Stern. San Diego Stock Center \#14021-0261.00 } \\
\text { Collected from Ivory Coast in } 1955\end{array}$ \\
\hline D. yakuba & 15.6 .8 & $\begin{array}{l}\text { Given by D. Matute. Isofemale stock, collected in São Tomé at } \\
\text { altitude } 110 \mathrm{~m} \text { in } 2009 \text { by D. Matute }\end{array}$ \\
\hline D. yakuba & yellow [1] & San Diego Species Stock Center \#14021-0261.05 \\
\hline D. yakuba & 4.23 .1 & $\begin{array}{l}\text { Given by D. Matute. Isofemale stock, collected in São Tomé at } \\
\text { altitude } 1070 \mathrm{~m} \text { in } 2009 \text { by D. Matute }\end{array}$ \\
\hline D. yakuba & LP1 & $\begin{array}{l}\text { Given by D. Matute. Isofemale stock, collected in São Tomé at } \\
\text { altitude } 0 \mathrm{~m} \text { in } 2009 \text { by D. Matute }\end{array}$ \\
\hline D. yakuba & 2.22 .1 & Given by D. Matute. Isofemale stock, collected in São Tomé at \\
\hline
\end{tabular}




\begin{tabular}{|c|c|c|}
\hline & & altitude $1250 \mathrm{~m}$ in 2009 by D. Matute \\
\hline D. yakuba & PB1.4.21 & $\begin{array}{l}\text { Given by D. Matute. Isofemale stock, collected in Bioko at } \\
\text { altitude } 1300 \mathrm{~m} \text { in } 2009 \text { by D. Matute }\end{array}$ \\
\hline D. santomea & SYN2005 & $\begin{array}{l}\text { Given by D. Matute. Mix of six isofemale lines collected by J. } \\
\text { Coyne at the field station Bom Sucesso (elevation } 1,150 \mathrm{~m} \text { ) in } \\
2005\end{array}$ \\
\hline D. santomea & STO.4 & $\begin{array}{l}\text { Given by D. Stern. San Diego Stock Center \#14021- } \\
0271.00 \\
\text { Collected in São Tomé in } 1998\end{array}$ \\
\hline D. santomea & Quija 650.22 & $\begin{array}{l}\text { Given by D. Matute. Isofemale stock, collected in São Tomé at } \\
\text { altitude } 650 \mathrm{~m} \text { in } 2009 \text { by D. Matute. }\end{array}$ \\
\hline D. santomea & Quija 650.37 & $\begin{array}{l}\text { Given by D. Matute. Isofemale stock, collected in São Tomé at } \\
\text { altitude } 650 \mathrm{~m} \text { in } 2005 \text { by Lucio Primo Monteiro under the } \\
\text { supervision of Daniel Lachaise. }\end{array}$ \\
\hline D. santomea & Quija 650.14 & $\begin{array}{l}\text { Given by D. Matute. Isofemale stock, collected in São Tomé at } \\
\text { altitude } 650 \mathrm{~m} \text { in } 2005 \text { by Lucio Primo Monteiro under the } \\
\text { supervision of Daniel Lachaise. }\end{array}$ \\
\hline D. santomea & BS14.1 & $\begin{array}{l}\text { Given by D. Matute. Isofemale stock, collected in São Tomé at } \\
\text { altitude } 1150 \mathrm{~m} \text { in } 2009 \text { by D. Matute }\end{array}$ \\
\hline D. santomea & CAR1490.3 & $\begin{array}{l}\text { Given by D. Matute. Isofemale stock, collected in São Tomé at } \\
\text { altitude } 1490 \mathrm{~m} \text { in } 2009 \text { by D. Matute }\end{array}$ \\
\hline D. santomea & B1300.13 & $\begin{array}{l}\text { Given by D. Matute. Isofemale stock, collected in São Tomé at } \\
\text { altitude } 1300 \mathrm{~m} \text { in } 2009 \text { by D. Matute }\end{array}$ \\
\hline D. santomea & OBAT1200.3 & $\begin{array}{l}\text { Given by D. Matute. Isofemale stock, collected in São Tomé at } \\
\text { altitude } 1200 \mathrm{~m} \text { in } 2009 \text { by D. Matute }\end{array}$ \\
\hline D. santomea & A1200.4 & $\begin{array}{l}\text { Given by D. Matute. Isofemale stock, collected in São Tomé at } \\
\text { altitude } 1200 \mathrm{~m} \text { in } 2009 \text { by D. Matute }\end{array}$ \\
\hline D. santomea & $\mathrm{C} 1350.14$ & $\begin{array}{l}\text { Given by D. Matute. Isofemale stock, collected in São Tomé at } \\
\text { altitude } 1350 \mathrm{~m} \text { in } 2009 \text { by D. Matute }\end{array}$ \\
\hline D. santomea & Rain42 & $\begin{array}{l}\text { Given by D. Matute. Isofemale stock, collected in São Tomé at } \\
\text { altitude } 1240 \mathrm{~m} \text { in } 2009 \text { by D. Matute }\end{array}$ \\
\hline D. santomea & Field3.4 & $\begin{array}{l}\text { Given by D. Matute. Isofemale stock, collected in São Tomé at } \\
\text { altitude } 1250 \mathrm{~m} \text { in } 2009 \text { by D. Matute }\end{array}$ \\
\hline D. teissieri & & Collected in Mt Selinda, Zimbabwe in 1970 \\
\hline D. teissieri & $\# 14021-0257.01$ & San Diego Stock Center \# 14021-0257.01 \\
\hline D. orena & & Isofemale stock collected by J. David in 1975 in Cameroon \\
\hline D. erecta & & Collected in La Lopé, Gabon by D. Lachaise in 2005 \\
\hline D. elegans & & $\begin{array}{l}\text { Given by B. Prud'homme. San Diego Stock Center \# 14027- } \\
\text { 0461.03. Collected in Hong-Kong. }\end{array}$ \\
\hline
\end{tabular}


Table S2. Hypandrial bristle number in pure species and F1 hybrids

${ }^{0}$ Flies raised at $25^{\circ} \mathrm{C},{ }^{1}$ Flies raised at $29^{\circ} \mathrm{C}$, ${ }^{2}$ Flies raised at $18{ }^{\circ} \mathrm{C}$, ${ }^{3}$ Flies raised at $21{ }^{\circ} \mathrm{C}$, ${ }^{4} \mathrm{Flies}$ raised at $14^{\circ} \mathrm{C},{ }^{5}$ Flies collected directly from the wild.

\begin{tabular}{|c|c|c|c|c|c|}
\hline & $\begin{array}{l}\text { Number } \\
\text { of Flies } \\
\text { with } 0 \\
\text { bristles }\end{array}$ & $\begin{array}{l}\text { Number } \\
\text { of Flies } \\
\text { with } 1 \\
\text { bristles }\end{array}$ & $\begin{array}{l}\text { Number } \\
\text { of Flies } \\
\text { with } 2 \\
\text { bristles }\end{array}$ & $\begin{array}{l}\text { Number } \\
\text { of Flies } \\
\text { with } 3 \\
\text { bristles }\end{array}$ & $\begin{array}{l}\text { Total } \\
\text { Number } \\
\text { of Flies }\end{array}$ \\
\hline \multicolumn{6}{|l|}{ D. melanogaster } \\
\hline $3870^{\circ}$ & 0 & 0 & 20 & 0 & 20 \\
\hline $3844^{0}$ & 0 & 0 & 21 & 0 & 21 \\
\hline $3841^{0}$ & 0 & 0 & 21 & 0 & 21 \\
\hline $3852^{0}$ & 0 & 0 & 21 & 0 & 21 \\
\hline $3864^{0}$ & 0 & 0 & 22 & 0 & 22 \\
\hline $\mathrm{T} .7^{0}$ & 0 & 0 & 20 & 0 & 20 \\
\hline T. $4^{0}$ & 0 & 0 & 19 & 1 & 20 \\
\hline $3875^{0}$ & 0 & 0 & 26 & 0 & 26 \\
\hline $3886^{0}$ & 0 & 0 & 19 & 1 & 20 \\
\hline T. $1^{0}$ & 0 & 0 & 19 & 1 & 20 \\
\hline $3839^{0}$ & 0 & 0 & 20 & 0 & 20 \\
\hline $3846^{0}$ & 0 & 0 & 20 & 0 & 20 \\
\hline $\operatorname{Sam}^{0}$ & 0 & 0 & 20 & 0 & 20 \\
\hline iso- $1^{0}$ & 0 & 6 & 7 & 1 & 15 \\
\hline Canton- $\mathrm{S}^{0}$ & 0 & 0 & 20 & 0 & 20 \\
\hline D. sechellia $G F P^{0}$ & 0 & 0 & 31 & 0 & 31 \\
\hline D. mauritiana ${ }^{0}$ & 0 & 0 & 34 & 0 & 34 \\
\hline D. mauritiana ${ }^{2}$ & 0 & 0 & 34 & 0 & 34 \\
\hline D. simulans 5 & 0 & 0 & 10 & 0 & 10 \\
\hline \multicolumn{6}{|l|}{ D. yakuba } \\
\hline Ivory Coast $^{0}$ & 0 & 0 & 32 & 0 & 32 \\
\hline Ivory Coast $^{2}$ & 0 & 0 & 30 & 0 & 30 \\
\hline $4.23 .1^{1}$ & 0 & 0 & 20 & 0 & 20 \\
\hline $\mathrm{PB} 1.4 .21^{1}$ & 0 & 0 & 25 & 0 & 25 \\
\hline $\mathrm{LP}^{1}$ & 0 & 0 & 35 & 0 & 35 \\
\hline $2.22 .1^{1}$ & 0 & 0 & 13 & 0 & 13 \\
\hline
\end{tabular}


$15.6 .8^{1}$

D. santomea

SYN $2005^{0}$

SYN2005

STO. $4^{0}$

Quija $650.37^{0}$

Quija 650.14

BS14.12

CAR $1490.3^{2}$

B1300.132

OBAT1200.3 $3^{2}$

A1200.4 $4^{2}$

C1350.14

Rain $42^{2}$

Field $3.4^{2}$

D. teissieri ${ }^{0}$

D. teissieri ${ }^{2}$

D. orena ${ }^{3}$

D. erecta $^{4}$

D. elegans $^{0}$

D. santomea/D. yakuba $\mathrm{F} 1$ hybrids $^{0}$

(D. santomea females x D. yakuba males)

D. santomea/D. yakuba $\mathrm{F} 1$ hybrids $^{0}$

(D. yakuba females x D. santomea males)

D. melanogaster/D. santomea hybrids

san/DC002 (1A1-1A1)

san/DC003 (1A1-1A1)

san/DC004 (1A1-1A3)

san/DC006 (1A3-1A8)

san/DC097 (1A7-1B4)

san/DC098 (1B2-1B8)

san/DC007 (1B1-1B5)

san/DC008 (1B4-1B10)

san/DC009 (1B9-1B13)

san/DC012 (1C3-1C5)

\begin{tabular}{|c|c|c|c|c|}
\hline 0 & 0 & 10 & 0 & 10 \\
\hline 60 & 0 & 0 & 0 & 60 \\
\hline 30 & 0 & 0 & 0 & 30 \\
\hline 29 & 0 & 0 & 0 & 29 \\
\hline 30 & 0 & 0 & 0 & 30 \\
\hline 35 & 0 & 0 & 0 & 35 \\
\hline 22 & 0 & 0 & 0 & 22 \\
\hline 21 & 0 & 0 & 0 & 21 \\
\hline 20 & 0 & 0 & 0 & 20 \\
\hline 22 & 0 & 0 & 0 & 22 \\
\hline 20 & 0 & 0 & 0 & 20 \\
\hline 20 & 0 & 0 & 0 & 20 \\
\hline 23 & 0 & 0 & 0 & 23 \\
\hline 21 & 0 & 0 & 0 & 21 \\
\hline 0 & 0 & 30 & 0 & 30 \\
\hline 0 & 0 & 30 & 0 & 30 \\
\hline 0 & 0 & 5 & 0 & 5 \\
\hline 0 & 0 & 10 & 0 & 10 \\
\hline 0 & 0 & 17 & 0 & 17 \\
\hline 0 & 2 & 32 & 0 & 34 \\
\hline 29 & 0 & 0 & 0 & 29 \\
\hline
\end{tabular}

\begin{tabular}{|c|c|c|c|c|}
\hline 0 & 0 & 10 & 0 & 10 \\
\hline 60 & 0 & 0 & 0 & 60 \\
\hline 30 & 0 & 0 & 0 & 30 \\
\hline 29 & 0 & 0 & 0 & 29 \\
\hline 30 & 0 & 0 & 0 & 30 \\
\hline 35 & 0 & 0 & 0 & 35 \\
\hline 22 & 0 & 0 & 0 & 22 \\
\hline 21 & 0 & 0 & 0 & 21 \\
\hline 20 & 0 & 0 & 0 & 20 \\
\hline 22 & 0 & 0 & 0 & 22 \\
\hline 20 & 0 & 0 & 0 & 20 \\
\hline 20 & 0 & 0 & 0 & 20 \\
\hline 23 & 0 & 0 & 0 & 23 \\
\hline 21 & 0 & 0 & 0 & 21 \\
\hline 0 & 0 & 30 & 0 & 30 \\
\hline 0 & 0 & 30 & 0 & 30 \\
\hline 0 & 0 & 5 & 0 & 5 \\
\hline 0 & 0 & 10 & 0 & 10 \\
\hline 0 & 0 & 17 & 0 & 17 \\
\hline 0 & 2 & 32 & 0 & 34 \\
\hline 29 & 0 & 0 & 0 & 29 \\
\hline
\end{tabular}

\begin{tabular}{|c|c|c|c|c|}
\hline 0 & 0 & 10 & 0 & 10 \\
\hline 60 & 0 & 0 & 0 & 60 \\
\hline 30 & 0 & 0 & 0 & 30 \\
\hline 29 & 0 & 0 & 0 & 29 \\
\hline 30 & 0 & 0 & 0 & 30 \\
\hline 35 & 0 & 0 & 0 & 35 \\
\hline 22 & 0 & 0 & 0 & 22 \\
\hline 21 & 0 & 0 & 0 & 21 \\
\hline 20 & 0 & 0 & 0 & 20 \\
\hline 22 & 0 & 0 & 0 & 22 \\
\hline 20 & 0 & 0 & 0 & 20 \\
\hline 20 & 0 & 0 & 0 & 20 \\
\hline 23 & 0 & 0 & 0 & 23 \\
\hline 21 & 0 & 0 & 0 & 21 \\
\hline 0 & 0 & 30 & 0 & 30 \\
\hline 0 & 0 & 30 & 0 & 30 \\
\hline 0 & 0 & 5 & 0 & 5 \\
\hline 0 & 0 & 10 & 0 & 10 \\
\hline 0 & 0 & 17 & 0 & 17 \\
\hline 0 & 2 & 32 & 0 & 34 \\
\hline 29 & 0 & 0 & 0 & 29 \\
\hline
\end{tabular}

\begin{tabular}{|c|c|c|c|c|}
\hline 0 & 0 & 10 & 0 & 10 \\
\hline 60 & 0 & 0 & 0 & 60 \\
\hline 30 & 0 & 0 & 0 & 30 \\
\hline 29 & 0 & 0 & 0 & 29 \\
\hline 30 & 0 & 0 & 0 & 30 \\
\hline 35 & 0 & 0 & 0 & 35 \\
\hline 22 & 0 & 0 & 0 & 22 \\
\hline 21 & 0 & 0 & 0 & 21 \\
\hline 20 & 0 & 0 & 0 & 20 \\
\hline 22 & 0 & 0 & 0 & 22 \\
\hline 20 & 0 & 0 & 0 & 20 \\
\hline 20 & 0 & 0 & 0 & 20 \\
\hline 23 & 0 & 0 & 0 & 23 \\
\hline 21 & 0 & 0 & 0 & 21 \\
\hline 0 & 0 & 30 & 0 & 30 \\
\hline 0 & 0 & 30 & 0 & 30 \\
\hline 0 & 0 & 5 & 0 & 5 \\
\hline 0 & 0 & 10 & 0 & 10 \\
\hline 0 & 0 & 17 & 0 & 17 \\
\hline 0 & 2 & 32 & 0 & 34 \\
\hline 29 & 0 & 0 & 0 & 29 \\
\hline
\end{tabular}

\begin{tabular}{|c|c|c|c|c|}
\hline 0 & 0 & 10 & 0 & 10 \\
\hline 60 & 0 & 0 & 0 & 60 \\
\hline 30 & 0 & 0 & 0 & 30 \\
\hline 29 & 0 & 0 & 0 & 29 \\
\hline 30 & 0 & 0 & 0 & 30 \\
\hline 35 & 0 & 0 & 0 & 35 \\
\hline 22 & 0 & 0 & 0 & 22 \\
\hline 21 & 0 & 0 & 0 & 21 \\
\hline 20 & 0 & 0 & 0 & 20 \\
\hline 22 & 0 & 0 & 0 & 22 \\
\hline 20 & 0 & 0 & 0 & 20 \\
\hline 20 & 0 & 0 & 0 & 20 \\
\hline 23 & 0 & 0 & 0 & 23 \\
\hline 21 & 0 & 0 & 0 & 21 \\
\hline 0 & 0 & 30 & 0 & 30 \\
\hline 0 & 0 & 30 & 0 & 30 \\
\hline 0 & 0 & 5 & 0 & 5 \\
\hline 0 & 0 & 10 & 0 & 10 \\
\hline 0 & 0 & 17 & 0 & 17 \\
\hline 0 & 2 & 32 & 0 & 34 \\
\hline 29 & 0 & 0 & 0 & 29 \\
\hline
\end{tabular}

\begin{tabular}{|c|c|c|c|c|}
\hline 0 & 0 & 10 & 0 & 10 \\
\hline 60 & 0 & 0 & 0 & 60 \\
\hline 30 & 0 & 0 & 0 & 30 \\
\hline 29 & 0 & 0 & 0 & 29 \\
\hline 30 & 0 & 0 & 0 & 30 \\
\hline 35 & 0 & 0 & 0 & 35 \\
\hline 22 & 0 & 0 & 0 & 22 \\
\hline 21 & 0 & 0 & 0 & 21 \\
\hline 20 & 0 & 0 & 0 & 20 \\
\hline 22 & 0 & 0 & 0 & 22 \\
\hline 20 & 0 & 0 & 0 & 20 \\
\hline 20 & 0 & 0 & 0 & 20 \\
\hline 23 & 0 & 0 & 0 & 23 \\
\hline 21 & 0 & 0 & 0 & 21 \\
\hline 0 & 0 & 30 & 0 & 30 \\
\hline 0 & 0 & 30 & 0 & 30 \\
\hline 0 & 0 & 5 & 0 & 5 \\
\hline 0 & 0 & 10 & 0 & 10 \\
\hline 0 & 0 & 17 & 0 & 17 \\
\hline 0 & 2 & 32 & 0 & 34 \\
\hline 29 & 0 & 0 & 0 & 29 \\
\hline
\end{tabular}

\begin{tabular}{|c|c|c|c|c|}
\hline 0 & 0 & 10 & 0 & 10 \\
\hline 60 & 0 & 0 & 0 & 60 \\
\hline 30 & 0 & 0 & 0 & 30 \\
\hline 29 & 0 & 0 & 0 & 29 \\
\hline 30 & 0 & 0 & 0 & 30 \\
\hline 35 & 0 & 0 & 0 & 35 \\
\hline 22 & 0 & 0 & 0 & 22 \\
\hline 21 & 0 & 0 & 0 & 21 \\
\hline 20 & 0 & 0 & 0 & 20 \\
\hline 22 & 0 & 0 & 0 & 22 \\
\hline 20 & 0 & 0 & 0 & 20 \\
\hline 20 & 0 & 0 & 0 & 20 \\
\hline 23 & 0 & 0 & 0 & 23 \\
\hline 21 & 0 & 0 & 0 & 21 \\
\hline 0 & 0 & 30 & 0 & 30 \\
\hline 0 & 0 & 30 & 0 & 30 \\
\hline 0 & 0 & 5 & 0 & 5 \\
\hline 0 & 0 & 10 & 0 & 10 \\
\hline 0 & 0 & 17 & 0 & 17 \\
\hline 0 & 2 & 32 & 0 & 34 \\
\hline 29 & 0 & 0 & 0 & 29 \\
\hline
\end{tabular}

\begin{tabular}{|c|c|c|c|c|}
\hline 0 & 0 & 10 & 0 & 10 \\
\hline 60 & 0 & 0 & 0 & 60 \\
\hline 30 & 0 & 0 & 0 & 30 \\
\hline 29 & 0 & 0 & 0 & 29 \\
\hline 30 & 0 & 0 & 0 & 30 \\
\hline 35 & 0 & 0 & 0 & 35 \\
\hline 22 & 0 & 0 & 0 & 22 \\
\hline 21 & 0 & 0 & 0 & 21 \\
\hline 20 & 0 & 0 & 0 & 20 \\
\hline 22 & 0 & 0 & 0 & 22 \\
\hline 20 & 0 & 0 & 0 & 20 \\
\hline 20 & 0 & 0 & 0 & 20 \\
\hline 23 & 0 & 0 & 0 & 23 \\
\hline 21 & 0 & 0 & 0 & 21 \\
\hline 0 & 0 & 30 & 0 & 30 \\
\hline 0 & 0 & 30 & 0 & 30 \\
\hline 0 & 0 & 5 & 0 & 5 \\
\hline 0 & 0 & 10 & 0 & 10 \\
\hline 0 & 0 & 17 & 0 & 17 \\
\hline 0 & 2 & 32 & 0 & 34 \\
\hline 29 & 0 & 0 & 0 & 29 \\
\hline
\end{tabular}

\begin{tabular}{|c|c|c|c|c|}
\hline 0 & 0 & 10 & 0 & 10 \\
\hline 60 & 0 & 0 & 0 & 60 \\
\hline 30 & 0 & 0 & 0 & 30 \\
\hline 29 & 0 & 0 & 0 & 29 \\
\hline 30 & 0 & 0 & 0 & 30 \\
\hline 35 & 0 & 0 & 0 & 35 \\
\hline 22 & 0 & 0 & 0 & 22 \\
\hline 21 & 0 & 0 & 0 & 21 \\
\hline 20 & 0 & 0 & 0 & 20 \\
\hline 22 & 0 & 0 & 0 & 22 \\
\hline 20 & 0 & 0 & 0 & 20 \\
\hline 20 & 0 & 0 & 0 & 20 \\
\hline 23 & 0 & 0 & 0 & 23 \\
\hline 21 & 0 & 0 & 0 & 21 \\
\hline 0 & 0 & 30 & 0 & 30 \\
\hline 0 & 0 & 30 & 0 & 30 \\
\hline 0 & 0 & 5 & 0 & 5 \\
\hline 0 & 0 & 10 & 0 & 10 \\
\hline 0 & 0 & 17 & 0 & 17 \\
\hline 0 & 2 & 32 & 0 & 34 \\
\hline 29 & 0 & 0 & 0 & 29 \\
\hline
\end{tabular}

\begin{tabular}{|c|c|c|c|c|}
\hline 0 & 0 & 10 & 0 & 10 \\
\hline 60 & 0 & 0 & 0 & 60 \\
\hline 30 & 0 & 0 & 0 & 30 \\
\hline 29 & 0 & 0 & 0 & 29 \\
\hline 30 & 0 & 0 & 0 & 30 \\
\hline 35 & 0 & 0 & 0 & 35 \\
\hline 22 & 0 & 0 & 0 & 22 \\
\hline 21 & 0 & 0 & 0 & 21 \\
\hline 20 & 0 & 0 & 0 & 20 \\
\hline 22 & 0 & 0 & 0 & 22 \\
\hline 20 & 0 & 0 & 0 & 20 \\
\hline 20 & 0 & 0 & 0 & 20 \\
\hline 23 & 0 & 0 & 0 & 23 \\
\hline 21 & 0 & 0 & 0 & 21 \\
\hline 0 & 0 & 30 & 0 & 30 \\
\hline 0 & 0 & 30 & 0 & 30 \\
\hline 0 & 0 & 5 & 0 & 5 \\
\hline 0 & 0 & 10 & 0 & 10 \\
\hline 0 & 0 & 17 & 0 & 17 \\
\hline 0 & 2 & 32 & 0 & 34 \\
\hline 29 & 0 & 0 & 0 & 29 \\
\hline
\end{tabular}

\begin{tabular}{|c|c|c|c|c|}
\hline 0 & 0 & 10 & 0 & 10 \\
\hline 60 & 0 & 0 & 0 & 60 \\
\hline 30 & 0 & 0 & 0 & 30 \\
\hline 29 & 0 & 0 & 0 & 29 \\
\hline 30 & 0 & 0 & 0 & 30 \\
\hline 35 & 0 & 0 & 0 & 35 \\
\hline 22 & 0 & 0 & 0 & 22 \\
\hline 21 & 0 & 0 & 0 & 21 \\
\hline 20 & 0 & 0 & 0 & 20 \\
\hline 22 & 0 & 0 & 0 & 22 \\
\hline 20 & 0 & 0 & 0 & 20 \\
\hline 20 & 0 & 0 & 0 & 20 \\
\hline 23 & 0 & 0 & 0 & 23 \\
\hline 21 & 0 & 0 & 0 & 21 \\
\hline 0 & 0 & 30 & 0 & 30 \\
\hline 0 & 0 & 30 & 0 & 30 \\
\hline 0 & 0 & 5 & 0 & 5 \\
\hline 0 & 0 & 10 & 0 & 10 \\
\hline 0 & 0 & 17 & 0 & 17 \\
\hline 0 & 2 & 32 & 0 & 34 \\
\hline 29 & 0 & 0 & 0 & 29 \\
\hline
\end{tabular}

\begin{tabular}{|c|c|c|c|c|}
\hline 0 & 0 & 10 & 0 & 10 \\
\hline 60 & 0 & 0 & 0 & 60 \\
\hline 30 & 0 & 0 & 0 & 30 \\
\hline 29 & 0 & 0 & 0 & 29 \\
\hline 30 & 0 & 0 & 0 & 30 \\
\hline 35 & 0 & 0 & 0 & 35 \\
\hline 22 & 0 & 0 & 0 & 22 \\
\hline 21 & 0 & 0 & 0 & 21 \\
\hline 20 & 0 & 0 & 0 & 20 \\
\hline 22 & 0 & 0 & 0 & 22 \\
\hline 20 & 0 & 0 & 0 & 20 \\
\hline 20 & 0 & 0 & 0 & 20 \\
\hline 23 & 0 & 0 & 0 & 23 \\
\hline 21 & 0 & 0 & 0 & 21 \\
\hline 0 & 0 & 30 & 0 & 30 \\
\hline 0 & 0 & 30 & 0 & 30 \\
\hline 0 & 0 & 5 & 0 & 5 \\
\hline 0 & 0 & 10 & 0 & 10 \\
\hline 0 & 0 & 17 & 0 & 17 \\
\hline 0 & 2 & 32 & 0 & 34 \\
\hline 29 & 0 & 0 & 0 & 29 \\
\hline
\end{tabular}

\begin{tabular}{|c|c|c|c|c|}
\hline 0 & 0 & 10 & 0 & 10 \\
\hline 60 & 0 & 0 & 0 & 60 \\
\hline 30 & 0 & 0 & 0 & 30 \\
\hline 29 & 0 & 0 & 0 & 29 \\
\hline 30 & 0 & 0 & 0 & 30 \\
\hline 35 & 0 & 0 & 0 & 35 \\
\hline 22 & 0 & 0 & 0 & 22 \\
\hline 21 & 0 & 0 & 0 & 21 \\
\hline 20 & 0 & 0 & 0 & 20 \\
\hline 22 & 0 & 0 & 0 & 22 \\
\hline 20 & 0 & 0 & 0 & 20 \\
\hline 20 & 0 & 0 & 0 & 20 \\
\hline 23 & 0 & 0 & 0 & 23 \\
\hline 21 & 0 & 0 & 0 & 21 \\
\hline 0 & 0 & 30 & 0 & 30 \\
\hline 0 & 0 & 30 & 0 & 30 \\
\hline 0 & 0 & 5 & 0 & 5 \\
\hline 0 & 0 & 10 & 0 & 10 \\
\hline 0 & 0 & 17 & 0 & 17 \\
\hline 0 & 2 & 32 & 0 & 34 \\
\hline 29 & 0 & 0 & 0 & 29 \\
\hline
\end{tabular}

\begin{tabular}{|c|c|c|c|c|}
\hline 0 & 0 & 10 & 0 & 10 \\
\hline 60 & 0 & 0 & 0 & 60 \\
\hline 30 & 0 & 0 & 0 & 30 \\
\hline 29 & 0 & 0 & 0 & 29 \\
\hline 30 & 0 & 0 & 0 & 30 \\
\hline 35 & 0 & 0 & 0 & 35 \\
\hline 22 & 0 & 0 & 0 & 22 \\
\hline 21 & 0 & 0 & 0 & 21 \\
\hline 20 & 0 & 0 & 0 & 20 \\
\hline 22 & 0 & 0 & 0 & 22 \\
\hline 20 & 0 & 0 & 0 & 20 \\
\hline 20 & 0 & 0 & 0 & 20 \\
\hline 23 & 0 & 0 & 0 & 23 \\
\hline 21 & 0 & 0 & 0 & 21 \\
\hline 0 & 0 & 30 & 0 & 30 \\
\hline 0 & 0 & 30 & 0 & 30 \\
\hline 0 & 0 & 5 & 0 & 5 \\
\hline 0 & 0 & 10 & 0 & 10 \\
\hline 0 & 0 & 17 & 0 & 17 \\
\hline 0 & 2 & 32 & 0 & 34 \\
\hline 29 & 0 & 0 & 0 & 29 \\
\hline
\end{tabular}

\begin{tabular}{|c|c|c|c|c|}
\hline 0 & 0 & 10 & 0 & 10 \\
\hline 60 & 0 & 0 & 0 & 60 \\
\hline 30 & 0 & 0 & 0 & 30 \\
\hline 29 & 0 & 0 & 0 & 29 \\
\hline 30 & 0 & 0 & 0 & 30 \\
\hline 35 & 0 & 0 & 0 & 35 \\
\hline 22 & 0 & 0 & 0 & 22 \\
\hline 21 & 0 & 0 & 0 & 21 \\
\hline 20 & 0 & 0 & 0 & 20 \\
\hline 22 & 0 & 0 & 0 & 22 \\
\hline 20 & 0 & 0 & 0 & 20 \\
\hline 20 & 0 & 0 & 0 & 20 \\
\hline 23 & 0 & 0 & 0 & 23 \\
\hline 21 & 0 & 0 & 0 & 21 \\
\hline 0 & 0 & 30 & 0 & 30 \\
\hline 0 & 0 & 30 & 0 & 30 \\
\hline 0 & 0 & 5 & 0 & 5 \\
\hline 0 & 0 & 10 & 0 & 10 \\
\hline 0 & 0 & 17 & 0 & 17 \\
\hline 0 & 2 & 32 & 0 & 34 \\
\hline 29 & 0 & 0 & 0 & 29 \\
\hline
\end{tabular}

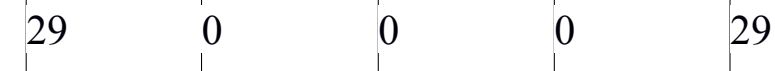

\begin{tabular}{|c|c|c|c|c|}
\hline 19 & 4 & 1 & 0 & 24 \\
\hline 15 & 7 & 2 & 0 & 24 \\
\hline 18 & 6 & 0 & 0 & 24 \\
\hline 18 & 3 & 3 & 0 & 24 \\
\hline 8 & 9 & 7 & 0 & 24 \\
\hline 19 & 3 & 2 & 0 & 24 \\
\hline 18 & 5 & 1 & 0 & 24 \\
\hline 21 & 2 & 1 & 0 & 24 \\
\hline 18 & 4 & 2 & 0 & 24 \\
\hline 17 & 5 & 2 & 0 & 24 \\
\hline
\end{tabular}




\begin{tabular}{|c|c|c|c|c|c|}
\hline san/DC099 (1C3-1C4) & 21 & 3 & 0 & 0 & 24 \\
\hline $\operatorname{san} / \mathrm{DC} 013(1 \mathrm{C} 4-1 \mathrm{D} 2)$ & 16 & 8 & 0 & 0 & 24 \\
\hline $\operatorname{san} / \mathrm{DC} 014$ (1D1-1D2) & 20 & 4 & 0 & 0 & 24 \\
\hline $\operatorname{san} / \mathrm{DC} 400(1 \mathrm{D} 2-1 \mathrm{E} 1)$ & 19 & 3 & 2 & 0 & 24 \\
\hline $\operatorname{san} / \mathrm{DC} 019$ (1E1-1E3) & 18 & 5 & 1 & 0 & 24 \\
\hline san/DC436 (1E3-1E5) & 22 & 0 & 2 & 0 & 24 \\
\hline $\operatorname{san} / \mathrm{DC} 401(2 \mathrm{~A} 2-2 \mathrm{~B} 1)$ & 21 & 3 & 0 & 0 & 24 \\
\hline san/TM3 (control) & 68 & 18 & 2 & 0 & 95 \\
\hline
\end{tabular}


Table. S3. achaete-scute mutant lines used.

Coordinates are for D. melanogaster reference genome iso-1 (FB2013_03, version 3). Based on restriction sites we estimated that point 0 of $[60,61]$ corresponds to position 330342 in iso-1. Compared to other D. melanogaster strains, iso-1 contains a 6127-bp transposable element named $3 \mathrm{~S} 18\{\} 4 / \mathrm{TE} 19523$ at position 322,507-328,633. Coordinates were adjusted to account for the shift due to the transposable element.

\begin{tabular}{|c|c|c|}
\hline Name & Origin and full genotype & Description \\
\hline$s c^{M 6}$ & $\begin{array}{l}\text { Bloomington Stock Center \#52668 } \\
s c[M 6] / F M 7 i, P\{w[+m C]=A c t G F P\} J M R 3\end{array}$ & Nonsense Glu114X mutation in scute. \\
\hline$a c^{C A M I}$ & $\begin{array}{l}\text { Bloomington Stock Center \#36540 } \\
y[1] P\{w[+m W . h s]=G a w B\} C G 32816[N P 6014] \\
\text { ac [cami] }\end{array}$ & $\begin{array}{l}\text { Deletion from } 263861 \text { to } 264099 \text {, which } \\
\text { includes nucleotides } 28 \text { to } 265 \text { of achaete } \\
\text { coding sequence, and insertion of } 21 \text { bp at the } \\
\text { same location (remains of an excised P- } \\
\text { element). }\end{array}$ \\
\hline$s c^{6}$ & $\begin{array}{l}\text { Bloomington Stock Center \#108 } \\
s c[6] w[a]\end{array}$ & $\begin{array}{l}\text { Deletion from between ca. } 315550 \text { and } \\
316484 \text { to between } 338537 \text { and } 341499 \text {. }\end{array}$ \\
\hline$a s e^{l}$ & $\begin{array}{l}\text { Bloomington Stock Center \#104 } \\
D f(1) \text { ase-1, sc[ase-1] pn[1]/C(1)DX, y[1]f[1] }\end{array}$ & $\begin{array}{l}\text { Deletion from between } 341500 \text { and } 343845 \\
\text { to between } 360269 \text { and } 360967 .\end{array}$ \\
\hline$s c^{5}$ & $\begin{array}{l}\text { Bloomington Stock Center \#178 } \\
y[1] s c[5]\end{array}$ & 1.2-kb deletion between 346503 and 347999 . \\
\hline$a c^{l}$ & $\begin{array}{l}\text { Bloomington Stock Center \#8715 } \\
y[1] a c[1] w[1118] ; P\{w[+m C]=G A L 4-a c .13\} 1\end{array}$ & $\begin{array}{l}\text { Deletion from ca. } 242000 \text { to between } 257781 \\
\text { and } 259255 \text {. }\end{array}$ \\
\hline$s c^{l}$ & $\begin{array}{l}\text { Bloomington Stock Center \#176 } \\
y[1] s c[1]\end{array}$ & Gypsy insertion between 322067 and 322068 . \\
\hline$a c^{s b m}$ & $\begin{array}{l}\text { Given by P. Simpson. } \\
\text { ac [sbm] }\end{array}$ & $\begin{array}{l}\text { P element insertion between } 264099 \text { and } \\
264100 \text {, after nucleotide } 27 \text { counting } 5^{\prime} \text { from } \\
\text { the achaete ATG. }\end{array}$ \\
\hline$a c^{H w-1}$ & $\begin{array}{l}\text { Bloomington Stock Center \#109 } \\
D f(1) s c 10-1, s c[10-1] / y[1] a c[H w-1] \\
\text { (this stock produces only [yellow] males) }\end{array}$ & $\begin{array}{l}\text { Gypsy insertion between } 264219 \text { and } 264671 . \\
\text { Transcription of achaete terminates within } \\
\text { Gypsy sequence. The resulting truncated } \\
\text { transcript is overabundant compared to } \\
\text { wildtype. }\end{array}$ \\
\hline$s c^{29}$ & $\begin{array}{l}\text { Bloomington Stock Center \#1442 } \\
\text { In(1)sc[29], sc[29] w[a] eag[sc29] }\end{array}$ & $\begin{array}{l}\text { Inversion. Left breakpoint is between } 336621 \\
\text { and } 337006 .\end{array}$ \\
\hline$a c^{l} s c^{l}$ & $\begin{array}{l}\text { Bloomington Stock Center \#4596 } \\
y[1] a c[1] s c[1] p n[1]\end{array}$ & See $a c^{l}$ and $s c^{l}$. \\
\hline$S c^{H}$ & $\begin{array}{l}\text { Bloomington Stock Center \#4055 } \\
C(1) D X, y[1] f[1] ; T(1 ; 4) s c[H], s c[H]\end{array}$ & $\begin{array}{l}\text { Transposition. Left breakpoint is between } \\
317578 \text { and } 318088 \text {. }\end{array}$ \\
\hline$s c^{9}$ & $\begin{array}{l}\text { DGRC Kyoto Stock Center \#102028 } \\
\operatorname{In}(1) s c[9], s c[9] w[a] f[1] B x[1]\end{array}$ & $\begin{array}{l}\text { Inversion. Left breakpoint is between } 318656 \\
\text { and } 320436 .\end{array}$ \\
\hline
\end{tabular}




\begin{tabular}{|c|c|c|}
\hline$s c^{S 2}$ & $\begin{array}{l}\text { Bloomington Stock Center \#3333 } \\
T(1 ; 2) s c[S 2], y[+] s c[S 2]: \operatorname{cn}[1] M(2) 53[1] /+ \text {; } \\
C y O\end{array}$ & $\begin{array}{l}\text { Translocation associated with a deletion } \\
\text { which is between } 3.3 \mathrm{~kb} \text { and } 4.4 \mathrm{~kb} \text {. Left } \\
\text { breakpoint of the deletion is between } 329,139 \\
\text { and } 333,308 \text {. Minimal extent of the deletion } \\
\text { is } 330,042-333,308 \text {. }\end{array}$ \\
\hline$s c^{7}$ & $\begin{array}{l}\text { Bloomington Stock Center \#723 } \\
D f(1) B / \operatorname{In}(1) s c[7], \operatorname{In}(1) A M, s c[7] p t g[4]\end{array}$ & $\begin{array}{l}\text { Inversion associated with a deletion which is } \\
\text { between } 2.5 \mathrm{~kb} \text { and } 3.1 \mathrm{~kb} \text {. Left breakpoint of } \\
\text { the inversion is } 37 \mathrm{~kb} 3 \text { ' of the sc structural } \\
\text { gene [62]. Minimal extent of the deletion is } \\
332225-336481 \text {. }\end{array}$ \\
\hline$a c^{3}$ & $\begin{array}{l}\text { Bloomington Stock Center \#36541 } \\
\operatorname{In}(1) a c[3], s c[10-1] \text { ac [3] } w[1] \text { sable }[1] / F M 7 i \text {, } \\
P\{w[+m C]=A c t G F P\} J M R 3\end{array}$ & $\begin{array}{l}\text { Inversion whose left breakpoint is between } \\
263169 \text { and } 264010\left(a c^{3}\right) \text { and nonsense } \\
\text { Glu163X mutation in scute }\left(s c^{10-1}\right) \text {. }\end{array}$ \\
\hline$s c^{4}$ & $\begin{array}{l}\text { Bloomington Stock Center \#789 } \\
\operatorname{In}(1) s c[4], y[1] s c[4] A B O-X[1]\end{array}$ & $\begin{array}{l}\text { Inversion. Left breakpoint is } 7 \mathrm{~kb} 3^{\prime} \text { of the } \\
\text { scute transcribed region. }\end{array}$ \\
\hline$s c^{8}$ & $\begin{array}{l}\text { Bloomington Stock Center \#842 } \\
T(1 ; 3) s c[260-15], s c[260-15] / F M 6 B[1] d m[1] \\
s c[8] y[31 d] \\
\text { (this stock produces only [Bar] males) }\end{array}$ & $\begin{array}{l}\text { Inversion. Left breakpoint is between } 275383 \\
\text { and } 276564 .\end{array}$ \\
\hline$a c^{1}$ & $\begin{array}{l}\text { DGRC Kyoto Stock Center \#107246 } \\
\text { (ordered from Bloomington Stock Center with } \\
\text { ancient number \#3822) } \\
D f(1) s c[19] / y[1] a c[1] ; D p(1 ; 2) s c[19] / \operatorname{In}(2 L) C y \text {, } \\
S[2] C y[1]\end{array}$ & $\begin{array}{l}\text { See also } a c^{l} \text {. Translocation. Left breakpoint is } \\
\text { ca. } 343845 \text {. }\end{array}$ \\
\hline
\end{tabular}


Table S4. Hypandrial bristle number in achaete-scute mutants

\begin{tabular}{|c|c|c|c|c|c|}
\hline & $\begin{array}{l}\text { Number } \\
\text { of Flies } \\
\text { with } 0 \\
\text { bristles }\end{array}$ & $\begin{array}{l}\text { Number } \\
\text { of Flies } \\
\text { with } 1 \\
\text { bristles }\end{array}$ & $\begin{array}{l}\text { Number } \\
\text { of Flies } \\
\text { with } 2 \\
\text { bristles }\end{array}$ & $\begin{array}{l}\text { Number } \\
\text { of Flies } \\
\text { with } 3 \\
\text { bristles }\end{array}$ & $\begin{array}{l}\text { Total } \\
\text { Number } \\
\text { of Flies }\end{array}$ \\
\hline \multicolumn{6}{|c|}{ Null Mutations (Coding) } \\
\hline$s c^{M 6}$ & 15 & 0 & 0 & 0 & 15 \\
\hline$a c^{C A M I}$ & 0 & 0 & 15 & 0 & 15 \\
\hline \multicolumn{6}{|c|}{ Deletions (Cis-Regulatory) } \\
\hline$s c^{6}$ & 16 & 0 & 0 & 0 & 16 \\
\hline$a s e^{l}$ & 0 & 3 & 12 & 1 & 16 \\
\hline$s c^{5}$ & 0 & 0 & 17 & 0 & 17 \\
\hline$a c^{l}$ & 0 & 0 & 10 & 0 & 10 \\
\hline \multicolumn{6}{|c|}{ Insertions } \\
\hline$s c^{l}$ & 17 & 0 & 0 & 0 & 17 \\
\hline$a c^{s b m}$ & 1 & 0 & 14 & 0 & 15 \\
\hline$a c^{H w-1}$ & 0 & 0 & 15 & 0 & 15 \\
\hline \multicolumn{6}{|c|}{ Complex changes (inversions, translocations, etc.) } \\
\hline$s c^{29}$ & 16 & 0 & 0 & 0 & 16 \\
\hline$a c^{l} s c^{1}$ & 15 & 0 & 0 & 0 & 15 \\
\hline$s c^{H}$ & 6 & 0 & 0 & 0 & 6 \\
\hline$s c^{9}$ & 15 & 0 & 0 & 0 & 15 \\
\hline$s c^{S 2}$ & 5 & 0 & 0 & 0 & 5 \\
\hline$s c^{7}$ & 14 & 0 & 0 & 0 & 14 \\
\hline$a c^{3} s c^{10-1}$ & 14 & 0 & 0 & 0 & 14 \\
\hline$s c^{4}$ & 13 & 0 & 0 & 0 & 13 \\
\hline$s c^{8}$ & 0 & 0 & 23 & 0 & 23 \\
\hline$a c^{l} s c^{19}$ & 0 & 0 & 8 & 0 & 8 \\
\hline
\end{tabular}


Table S5. Test of various $U A S$-reporter constructs with $D C-G A L 4$

The number of adults (males and females) with singed/forked/absent dorsocentral thoracic bristles is shown for various $U A S-R N A i$ reporter lines. The phenotype was recorded individually for each of the four dorsocentral bristles: AL: anterior left, AR: anterior right, PL: posterior left, PR: posterior right. Dcr2: UAS-Dicer-2, WT: wild-type bristle phenotype. Crosses were performed at $25^{\circ} \mathrm{C}$ and at $29^{\circ} \mathrm{C}$ for each genotype.

\begin{tabular}{|c|c|c|c|c|c|c|c|c|c|c|c|c|c|c|c|c|c|c|}
\hline \begin{tabular}{|l|} 
Bristles \\
displaying a \\
mutant \\
phenotype
\end{tabular} & & \begin{tabular}{|l|} 
AL \\
AR \\
PL \\
PR
\end{tabular} & $\begin{array}{l}\text { AL } \\
\text { AR } \\
\text { PL }\end{array}$ & $\begin{array}{l}\text { AL } \\
\text { AR } \\
\text { PR }\end{array}$ & $\begin{array}{l}\text { AL } \\
\text { PL } \\
\text { PR }\end{array}$ & $\begin{array}{l}\text { AR } \\
\text { PL } \\
\text { PR }\end{array}$ & $\begin{array}{l}\mathbf{A L} \\
\mathbf{A R}\end{array}$ & $\begin{array}{l}\text { PL } \\
\text { PR }\end{array}$ & $\begin{array}{l}\mathbf{A L} \\
\mathbf{P L}\end{array}$ & $\begin{array}{l}\mathbf{A R} \\
\mathbf{P R}\end{array}$ & $\begin{array}{l}\text { AL } \\
\text { PR }\end{array}$ & $\begin{array}{l}\text { AR } \\
\text { PL }\end{array}$ & $\mathbf{A L}$ & AR & PL & PR & WT & $\begin{array}{c}\text { Total } \\
\text { Number of } \\
\text { Flies }\end{array}$ \\
\hline \multirow{2}{*}{$\begin{array}{l}\text { Dcr2; } \\
\text { singed }\end{array}$} & $25^{\circ} \mathrm{C}$ & 22 & 20 & 22 & & & 12 & & 1 & 7 & & & 1 & 3 & & & 8 & 88 \\
\hline & $29^{\circ} \mathrm{C}$ & 69 & 11 & 13 & & & 3 & & & & & & & 1 & & & 1 & 98 \\
\hline \multirow{2}{*}{$\begin{array}{l}\text { Der } 2 ; \\
\text { forked }^{103813}\end{array}$} & $25^{\circ} \mathrm{C}$ & 15 & 18 & 22 & & & 14 & 2 & 2 & 2 & & & 3 & 6 & 5 & 6 & 44 & 139 \\
\hline & $29^{\circ} \mathrm{C}$ & 123 & 33 & 36 & & & 15 & & 1 & 1 & & & & & & 1 & 5 & 215 \\
\hline \multirow{2}{*}{$\begin{array}{l}\text { Dcr } 2 ; \\
\text { forked }^{33200}\end{array}$} & $25^{\circ} \mathrm{C}$ & & 48 & & & & & & & & & & 3 & 3 & & & 46 & 100 \\
\hline & $29^{\circ} \mathrm{C}$ & 64 & 28 & 22 & & & 61 & & 1 & & & & 3 & 2 & & & 3 & 183 \\
\hline \multirow{2}{*}{$\begin{array}{l}\text { Dcr2; } \\
\text { forked }^{24632}\end{array}$} & $25^{\circ} \mathrm{C}$ & & & & & & & & & & & & & 1 & & & 154 & 155 \\
\hline & $29^{\circ} \mathrm{C}$ & & & & & & & & 1 & & & & & & 1 & & 175 & 177 \\
\hline \multirow{2}{*}{$\begin{array}{l}\text { Dcr2; } \\
\text { singed }^{32579}\end{array}$} & $25^{\circ} \mathrm{C}$ & & & & & & & & & & & & & & & & 151 & 151 \\
\hline & $29^{\circ} \mathrm{C}$ & & & & & & & & & & & & & & & & 217 & 217 \\
\hline \multirow{2}{*}{$\begin{array}{l}\text { Dcr2; } \\
a c^{100647}\end{array}$} & $25^{\circ} \mathrm{C}$ & & & & & & & & & & & & & & & & 136 & 136 \\
\hline & $29^{\circ} \mathrm{C}$ & & & & & & & & & & & & & & & & 258 & 258 \\
\hline \multirow{2}{*}{$\begin{array}{l}\text { Dcr 2; } \\
\text { sc }^{105951}\end{array}$} & $25^{\circ} \mathrm{C}$ & & & & & & & & & & & & & & & & 77 & 77 \\
\hline & $29^{\circ} \mathrm{C}$ & & & & & & & & & & & & & & & & 113 & 113 \\
\hline
\end{tabular}


Table S6. Achaete-scute GAL4 lines and their hypandrial bristle phenotype with Dcr2; UASsinged.RNAi ${ }^{105747}$.

Lines are ordered according to their left coordinates (Release 5). The origin of each GAL4 line is indicated by a letter code: V: Vienna Drosophila Research Center [63], J: Janelia Farm [38], S: This Study. The orientation of the scute cis-regulatory region within the reporter construct is indicated by + or -. N: no data, I: determined by PCR in this study. In bold are the 3 GAL4 lines which produce a hypandrial bristle mutant phenotype. For short, GMR15E09, GMR18C05 and VT054839 are named $15 E 09,18 C 05$ and 054839 in the main text, respectively. Comparison of the VT085439 cis-regulatory element from $D$. santomea and $D$. yakuba revealed no difference when tested in GAL4 reporter lines with Dcr2; UAS-singed.RNAi ${ }^{105747}$ (last two columns).

\begin{tabular}{|c|c|c|c|c|c|c|c|}
\hline Name & Origin & $\begin{array}{l}\text { Orien } \\
\text { tation }\end{array}$ & Coordinates & $\begin{array}{l}\text { Number of } \\
\text { Flies with } 0 \\
\text { singed } \\
\text { bristles }\end{array}$ & $\begin{array}{l}\text { Number of } \\
\text { Flies with } 1 \\
\text { singed } \\
\text { bristles }\end{array}$ & $\begin{array}{l}\text { Number of } \\
\text { Flies with } 2 \\
\text { singed } \\
\text { bristles }\end{array}$ & $\begin{array}{l}\text { Total } \\
\text { Number } \\
\text { of Flies }\end{array}$ \\
\hline VT054793 & $\mathrm{V}$ & - & $X: 252,196-250,097$ & 5 & 0 & 0 & 5 \\
\hline VT054794 & V & + & $X: 251,577-253,760$ & 5 & 0 & 0 & 5 \\
\hline VT054795 & $\mathrm{V}$ & + & $X: 255,238-257,329$ & 5 & 0 & 0 & 5 \\
\hline VT054796 & $\mathrm{V}$ & + & $X: 256,878-259,056$ & 5 & 0 & 0 & 5 \\
\hline VT054798 & $\mathrm{V}$ & - & $X: 262,456-260,258$ & 5 & 0 & 0 & 5 \\
\hline VT054799 & $\mathrm{V}$ & + & $X: 261,978-264,142$ & 5 & 0 & 0 & 5 \\
\hline GMR14C10 & $\mathrm{J}$ & $\mathrm{N}$ & $X: 265,138-267,274$ & 5 & 0 & 0 & 5 \\
\hline GMR15B10 & $\mathrm{J}$ & $\mathrm{N}$ & $X: 266,123-269,675$ & 5 & 0 & 0 & 5 \\
\hline GMR15C11 & $\mathrm{J}$ & $\mathrm{N}$ & $X: 269,057-272,751$ & 5 & 0 & 0 & 5 \\
\hline GMR15X09 & $\mathrm{S}$ & + & $X: 271,792-275,220$ & 5 & 0 & 0 & 5 \\
\hline VT054805 & $\mathrm{V}$ & + & $X: 273,824-275,941$ & 5 & 0 & 0 & 5 \\
\hline GMR15A01 & $\mathrm{J}$ & $\mathrm{N}$ & $X: 277,456-280,802$ & 5 & 0 & 0 & 5 \\
\hline GMR14C12 & $\mathrm{J}$ & $\mathrm{N}$ & $X: 276,489-278,649$ & 5 & 0 & 0 & 5 \\
\hline GMR15A04 & $\mathrm{J}$ & $\mathrm{N}$ & $X: 277,456-280,802$ & 6 & 0 & 0 & 6 \\
\hline GMR15C10 & $\mathrm{J}$ & $\mathrm{N}$ & $X: 280,274-283,966$ & 5 & 0 & 0 & 5 \\
\hline GMR15E07 & $\mathrm{J}$ & $\mathrm{N}$ & $X: 282,747-286,633$ & 5 & 0 & 0 & 5 \\
\hline GMR15E09 & $\mathbf{J}$ & - & $X: 289,793-285,861$ & $\mathbf{0}$ & $\mathbf{0}$ & 6 & 6 \\
\hline GMR13D04 & $\mathrm{J}$ & $\mathrm{N}$ & $X: 291,606-295,262$ & 5 & 0 & 0 & 5 \\
\hline GMR13C08 & $\mathrm{J}$ & $\mathrm{N}$ & $X: 294,631-298,184$ & 5 & 0 & 0 & 5 \\
\hline GMR12H02 & $\mathrm{J}$ & $\mathrm{N}$ & $X: 297,331-300,499$ & 5 & 0 & 0 & 5 \\
\hline GMR13B12 & $\mathrm{J}$ & $\mathrm{N}$ & $X: 299,747-303,236$ & 6 & 0 & 0 & 6 \\
\hline
\end{tabular}




\begin{tabular}{|c|c|c|c|c|c|c|c|}
\hline VT054820 & V & - & $X: 303,848-301,726$ & 6 & 0 & 0 & 6 \\
\hline VT054821 & $\mathrm{V}$ & - & $X: 306,956-304,793$ & 6 & 0 & 0 & 6 \\
\hline VT054822b & $\mathrm{S}$ & $\mathrm{N}$ & $X: 308,704-306,743$ & 7 & 0 & 0 & 7 \\
\hline VT054823 & V & + & $X: 308,224-310,380$ & 9 & 0 & 0 & 9 \\
\hline VT054824 & V & - & $X: 312,108-310,003$ & 7 & 0 & 0 & 7 \\
\hline VT054825 & V & + & $X: 311,767-313,924$ & 6 & 0 & 0 & 6 \\
\hline VT054826 & V & - & $X: 315,628-313,523$ & 6 & 0 & 0 & 6 \\
\hline VT054827 & V & + & $X: 315,231-317,337$ & 7 & 0 & 0 & 7 \\
\hline VT054828 & $\mathrm{V}$ & - & $X: 319,149-316,931$ & 15 & 0 & 0 & 15 \\
\hline VT054829 & $\mathrm{V}$ & - & $X: 320,924-318,733$ & 12 & 0 & 0 & 12 \\
\hline VT054831 & $\mathrm{V}$ & + & $X: 328,588-330,249$ & 8 & 0 & 0 & 8 \\
\hline VT054832 & $\mathrm{V}$ & - & $X: 333,580-331,369$ & 6 & 0 & 0 & 6 \\
\hline GMR18G09 & $\mathrm{J}$ & $\mathrm{N}$ & $X: 332,537-335,588$ & 5 & 0 & 0 & 5 \\
\hline VT054833 & V & - & $X: 335,283-333,110$ & 7 & 0 & 0 & 7 \\
\hline GMR18E07 & $\mathrm{J}$ & $\mathrm{N}$ & $X: 334,502-337,059$ & 5 & 0 & 0 & 5 \\
\hline VT054834 & $\mathrm{V}$ & + & $X: 334,894-337,095$ & 6 & 0 & 0 & 6 \\
\hline GMR19D04 & $\mathrm{J}$ & $+/ \mathrm{I}$ & $X: 336,372-340,205$ & 5 & 0 & 0 & 5 \\
\hline VT054835 & $\mathrm{V}$ & + & $X: 336,673-338,747$ & 7 & 0 & 0 & 7 \\
\hline VT054836 & $\mathrm{V}$ & - & $X: 340,523-338,435$ & 5 & 0 & 0 & 5 \\
\hline GMR18C05 & $\mathbf{J}$ & $-/ \mathbf{I}$ & $X: 341,372-339,336$ & $\mathbf{0}$ & $\mathbf{0}$ & 6 & 6 \\
\hline GMR19B11 & $\mathrm{J}$ & $+/ \mathrm{I}$ & $X: 340,180-343,911$ & 6 & 0 & 0 & 6 \\
\hline VT054838 & $\mathrm{V}$ & + & $X: 341,960-344,040$ & 5 & 0 & 0 & 5 \\
\hline GMR18G07 & $\mathrm{J}$ & $+/ \mathrm{I}$ & $X: 343,240-346,246$ & 6 & 0 & 0 & 6 \\
\hline VT054839 & $\mathbf{V}$ & + & $X: 343,684-345,799$ & $\mathbf{0}$ & $\mathbf{0}$ & 5 & 5 \\
\hline GMR18F05 & $\mathrm{J}$ & $\mathrm{N}$ & $X: 345,467-348,263$ & 5 & 0 & 0 & 5 \\
\hline VT054840 & $\mathrm{V}$ & - & $X: 347,574-345,470$ & 5 & 0 & 0 & 5 \\
\hline VT054841 & $\mathrm{V}$ & - & X: 349,308- 347,221 & 7 & 0 & 0 & 7 \\
\hline GMR19A06 & $\mathrm{J}$ & $\mathrm{N}$ & $X: 347,473-350,875$ & 5 & 0 & 0 & 5 \\
\hline VT054842 & $\mathrm{V}$ & - & $X: 351,081-348,769$ & 5 & 0 & 0 & 5 \\
\hline GMR18E10 & $\mathrm{J}$ & $\mathrm{N}$ & $X: 349,909-352,526$ & 5 & 0 & 0 & 5 \\
\hline VT054843 & $\mathrm{V}$ & - & $X: 352,807-350,712$ & 7 & 0 & 0 & 7 \\
\hline GMR20B05 & $\mathrm{J}$ & $\mathrm{N}$ & $X: 351,638-353,720$ & 5 & 0 & 0 & 5 \\
\hline VT054845 & $\mathrm{V}$ & + & $X: 357,132-359,309$ & 5 & 0 & 0 & 5 \\
\hline VT054846 & $\mathrm{V}$ & - & X: 360,191- 358,098 & 8 & 0 & 0 & 8 \\
\hline
\end{tabular}


bioRxiv preprint doi: https://doi.org/10.1101/313197; this version posted July 16,2018 . The copyright holder for this preprint (which was not certified by peer review) is the author/funder. All rights reserved. No reuse allowed without permission.

\begin{tabular}{|l|l|l|l|l|l|l|}
\hline $\begin{array}{l}\text { VT054839mel S } \\
\text {-BL2057 }\end{array}$ & + & Does not apply. & 1 & 2 & 2 & 5 \\
\hline VT054839yak S & & & & & & \\
\hline VT054839san & $S$ & Does not apply. & 7 & 4 & 7 & 18 \\
\hline
\end{tabular}


Table S7. Primers used.

All primers were purchased from Sigma Aldrich.

\begin{tabular}{|c|c|}
\hline Name & Sequence \\
\hline $15 \mathrm{X} 09 \mathrm{FF}$ & CACCTT TCG CGA TGC AGG GAG AAA GTG A \\
\hline 15X09RR & ATG CTG CGC AGC GTG AGA AAA TGC A \\
\hline VT054822bisF & CACCAAC GGA TTG GCG TGT TAA CCA GCG A \\
\hline VT054822bisR & AAG CGG AAG AGG TAT CGG TGT CCT T \\
\hline VT054839FF & CACCGGCAACTTTCAGCTCAGTTATGTCA \\
\hline VT054839RR & CAT CGT CGC AGC GGG AAT ATG AAT T \\
\hline 18C05FDsanyak & CACCGAGTGAGTGGACCGCTATTTGTACA \\
\hline 18C05Rsanyak & GAGCTGTTTGCTATCGGGGTGCGA \\
\hline 18C05FDmel & CACCGAATGAGTGGACCGCTATTTGTACA \\
\hline $18 \mathrm{C} 05 \mathrm{Rmel}$ & CAAGCCGTTTTCCTTTGAGGTGTCT \\
\hline $18 \mathrm{C} 05 \mathrm{bDF}$ & CACCCGAGTGCAGACACACAGACAGGTCT \\
\hline $18 \mathrm{C} 05 \mathrm{dDF}$ & CACCGTTCGCGGACCTAAGCCGCTTAAGT \\
\hline $18 \mathrm{C} 05 \mathrm{aR}$ & TTG AGT TCC CCT TGG CCG CTG TGA A \\
\hline $18 \mathrm{C} 05 \mathrm{cR}$ & TCT GCT GCT CCC GTT GAT GGT GTT G \\
\hline $18 \mathrm{C} 05 \mathrm{bF} 2$ & CACCTG AAA ACG GAT CGA AAC GGC GAG T \\
\hline $18 \mathrm{C} 05 \mathrm{cR} 2$ & GGT GTT GAT TGT GCG CCA GTC AAG T \\
\hline $18 \mathrm{C} 05 \mathrm{aR} 2 \mathrm{mel}$ & AAGCAGCTAAGCCTAGAACCCTGTTAC \\
\hline Scute-CDS-FOR & GGTACCTGTTGATCGTTMTCCGGAA \\
\hline Scute-CDS-FOR & AAGCTTAGTCACTGCTCCTGCCATAG \\
\hline VT054838 forward & СТCCCCAGACTAACCCACTC \\
\hline VT054838 reverse & AGCACAAAAAGGGCAGGAAC \\
\hline GAL4 forward & CGAACAAGCATGCGATATTT \\
\hline GAL4 reverse & GCTGCCGAGTCAATCGATAC \\
\hline VT054839 forward & GGTGCATCCATCCATTCCT \\
\hline VT054839 reverse & GCGGGAATATGAATTTTATGTAGA \\
\hline Gal4 lines sequencing forward & ACAAGTTTGTACAAAAAAGCAGGCT \\
\hline VT054836 forward & GACCTATGCACCAGCCATTT \\
\hline VT054836 reverse & CGCACCGGACTTTTAATTTG \\
\hline GMR15E09 forward & GGTGGACTCCGTTTCAGGT \\
\hline GMR15E09 reverse & GCCGAACGGCAATAAAGTAA \\
\hline
\end{tabular}




\begin{tabular}{|c|c|}
\hline PBPGUw-GIBSON-For & ATAGGGGTTCCGCGCACAT \\
\hline PBPGUw-GIBSON-Rev & CTTTTATACCGCTGCGCTCGAT \\
\hline San18C05_1-1070-Rev & ATTGCCAACTGTCCAAGAAGTC \\
\hline Yak18C05_1021-2085-For & AAAGGAATTGTACGCAGCCAAG \\
\hline San18C05_1-1609-Rev & TTCGGCCAAAAATTGATGAG \\
\hline Yak18C05_1571-2085-For & TGGCATTTGTTGTTCTCTGTG \\
\hline Yak18C05_1-550-Rev & TAATTTGGGCCCCGTGGA \\
\hline San18C05_500-2079-For & TTTATTTATTTTGCGGACTCAGA \\
\hline San18C05_1-550-Rev & ACCGGACTTTTAATTTGGGCCCCGTGGA \\
\hline Yak18C05_551-2085-For & GCCCAAATTAAAAGTCCGGTGCGCCCAA \\
\hline Yak18C05_1-1550-Rev_1 & AGGTAATCAAGTCACATTGCAGTTGTTTGC \\
\hline San18C05_1489-2079-For & GCAATGTGACTTGATTACCTCATTTAATTGAATG \\
\hline Yak_1078-2086_San-SNP970-Rev & acctggctctTAACTAAATTGCTGCCCCGAAC \\
\hline Yak_1-1078_San-SNP970-For & caatttagttAAGAGCCAGGTACGCGCTA \\
\hline Yak_1535-2086_San-SNP1429-Rev & ccaaagaaggcaaCATTTATTTCCGTGATACAACTAAAGCTG \\
\hline Yak_1-1535_San-SNP1429-For & ggaaataaatGCTGCCTTCTTTGGAAATAGTAG \\
\hline Yak_1613-2086-San-SNP1507-Rev & aattaaatgaggCAATCAAGTCACATTGCAGTTGTTTACC \\
\hline Yak_1-1613-San-SNP1507-For & gtgacttgattGCCTCATTTAATTGAATGTTTAACTC \\
\hline Yak_1880-2086-San-SNP1775-Rev & caaagtcaaataCAAGAAACGGTACATTGGTGGC \\
\hline Yak_1-1880-San-SNP1775-For & taccgtttcttGTATTTGACTTTGTTACGCCC \\
\hline San-Yak_lines_sequencing-For & AATGGGTCACCCCGTGTA \\
\hline San-Yak_lines_sequencing-Rev & GGTCTCTCAGGGTTTTCAAGC \\
\hline EMSA_Yak_1775-For & GTACCGTTTCTTTTATTTGACTTTG \\
\hline EMSA_San_T1775G-For & GTACCGTTTCTTGTATTTGACTTTG \\
\hline EMSA_1775-Rev & TCTTGGTTATTATTCCGAGGTG \\
\hline 18C05_Ancestral_Gibson-for & $\begin{array}{l}\text { TTCCCCGAAAAGTGCCACCTGACGTCTAAGAAACCATT } \\
\text { ATTATCATGACATTAACCTATAAAAATAGGCGTATCACGA } \\
\text { GGCCCTTTCGTCTTCAAGAATTCGTTTATCACAAGTTTG }\end{array}$ \\
\hline 18C05_Ancestral_Gibson-rev & $\begin{array}{l}\text { TCGATCCCCGGGCGAGCTCGGCCGGCCGTTTATCACCA } \\
\text { CTTTG }\end{array}$ \\
\hline
\end{tabular}

Table S8. PCR fragments cloned into pENTR/D-TOPO

\begin{tabular}{|l|lll|}
\hline Fragment & Template & Forward primer & Reverse primer \\
\hline 15 X09 & D. melanogaster iso-1 & 15X09FF & 15X09RR \\
\hline
\end{tabular}




\begin{tabular}{|c|c|c|c|}
\hline VT054822b & D. melanogaster iso-1 & VT054822bisF & VT054822bisR \\
\hline VT054839yak & D. yakuba Ivory Coast & VT054839FF & VT054839RR \\
\hline VT054839san & D. santomea SYN2005 & VT054839FF & VT054839RR \\
\hline 18C05_T7 & D. melanogaster T7 & 18C05FDmel & 18C05Rmel \\
\hline 18C05_BL2057 & D. melanogaster iso-1 & 18C05FDmel & $18 \mathrm{C} 05 \mathrm{Rmel}$ \\
\hline 18C05Yakfull & D. yakuba Ivory Coast & 18C05FDsanyak & 18C05Ryaksan \\
\hline 18C05Sanfull & D. santomea SYN2005 & 18C05FDsanyak & 18C05Ryaksan \\
\hline 18C05AmelBL & D. melanogaster iso-1 & 18C05FDmel & $18 \mathrm{C} 05 \mathrm{aR} 2 \mathrm{mel}$ \\
\hline 18C05BmelBL & D. melanogaster iso-1 & $18 \mathrm{C} 05 \mathrm{bF} 2$ & $18 \mathrm{C} 05 \mathrm{cR} 2$ \\
\hline $18 \mathrm{C} 05 \mathrm{CmelBL}$ & D. melanogaster iso-1 & $18 \mathrm{C} 05 \mathrm{dDF}$ & 18C05Rmel \\
\hline $18 \mathrm{C} 05 \mathrm{ABmelBL}$ & D. melanogaster iso-1 & 18C05FDmel & $18 \mathrm{CO5} \mathrm{cR}$ \\
\hline 18C05BCmelBL & D. melanogaster iso-1 & $18 \mathrm{C} 05 \mathrm{bDF}$ & 18C05Rmel \\
\hline 18C05Asan & D. santomea SYN2005 & 18C05FDsanyak & $18 \mathrm{C} 05 \mathrm{aR}$ \\
\hline 18C05Bsan & D. santomea SYN2005 & $18 \mathrm{C} 05 \mathrm{bDF}$ & $18 \mathrm{C} 05 \mathrm{cR} 2$ \\
\hline 18C05Ayak & D. yakuba Ivory Coast & 18C05FDsanyak & $18 \mathrm{C} 05 \mathrm{aR}$ \\
\hline 18C05Byak & D. yakuba Ivory Coast & $18 \mathrm{C} 05 \mathrm{bF} 2$ & $18 \mathrm{C} 05 \mathrm{cR} 2$ \\
\hline 18C05Cyak & D. yakuba Ivory Coast & $18 \mathrm{C} 05 \mathrm{dDF}$ & 18C05Ryaksan \\
\hline $\begin{array}{l}\text { VT054839mel- } \\
\text { BL2057 }\end{array}$ & D. melanogaster iso-1 & VT054839FF & VT054839RR \\
\hline VT054839yak & D. yakuba Ivory Coast & VT054839FF & VT054839RR \\
\hline VT054839san & D. santomea SYN2005 & VT054839FF & VT054839RR \\
\hline
\end{tabular}


Table S9. PCR fragments used for Gibson assembly into pBPSUw

\begin{tabular}{|c|c|c|c|c|}
\hline \# & Fragment name & Template & Forward primer & Reverse primer \\
\hline 1. & San_1-1500 & $\begin{array}{l}\text { 18C05_san_full- } \\
\text { pBPSUw }\end{array}$ & PBPGUw-GIBSON-For & $\begin{array}{l}\text { San18C05_1-1609bp- } \\
\operatorname{Rev}\end{array}$ \\
\hline 2. & Yak_1500-2085 & $\begin{array}{l}\text { 18C05_yak_full- } \\
\text { pBPSUw }\end{array}$ & Yak18C05_1571-2085-For & PBPGUw-GIBSON-Rev \\
\hline 3. & Yak_1-1000 & $\begin{array}{l}\text { 18C05_yak_full- } \\
\text { pBPSUw }\end{array}$ & PBPGUw-GIBSON-For & San18C05_1-1070-Rev \\
\hline 4. & San_1000-2085 & $\begin{array}{l}\text { 18C05_san_full- } \\
\text { pBPSUw }\end{array}$ & Yak18C05_1021-2085-For & PBPGUw-GIBSON-Rev \\
\hline 5. & $\begin{array}{l}\text { San_1- } \\
1000+\text { Yak } 1001-1500\end{array}$ & $\begin{array}{l}\text { San1-1000+Yak1001- } \\
\text { 2085-pBPGUw }\end{array}$ & PBPGUw-GIBSON-For & San18C05_1-1609-Rev \\
\hline 6. & San_1500-2079 & $\begin{array}{l}\text { 18C05_san_full- } \\
\text { pBPSUw }\end{array}$ & Yak18C05_1571-2085-For & PBPGUw-GIBSON-Rev \\
\hline 7. & San_1-500 & $\begin{array}{l}\text { 18C05_san_full- } \\
\text { pBPSUw }\end{array}$ & PBPGUw-GIBSON-For & San18C05_1-550-Rev \\
\hline 8. & Yak_500-2085 & $\begin{array}{l}\text { 18C05_yak_full- } \\
\text { pBPSUw }\end{array}$ & Yak18C05_551-2085-For & PBPGUw-GIBSON-Rev \\
\hline 9. & Yak_1-1500 & $\begin{array}{l}\text { 18C05_yak_full- } \\
\text { pBPSUw }\end{array}$ & PBPGUw-GIBSON-For & $\begin{array}{l}\text { Yak18C05_1-1550- } \\
\text { Rev_1 }\end{array}$ \\
\hline 10. & San_1500-2079 & $\begin{array}{l}\text { 18C05_san_full- } \\
\text { pBPSUw }\end{array}$ & San18C05_1489-2079-For & PBPGUw-GIBSON-Rev \\
\hline 11. & San_1-1000 & $\begin{array}{l}\text { 18C05_san_full- } \\
\text { pBPSUw }\end{array}$ & PBPGUw-GIBSON-For & San18C05_1-1070-Rev \\
\hline 12. & Yak_1001-2085 & $\begin{array}{l}\text { 18C05_yak_full- } \\
\text { pBPSUw }\end{array}$ & Yak18C05_1021-2085-For & PBPGUw-GIBSON-Rev \\
\hline 13. & $\begin{array}{l}\text { San_1001- } \\
1500+\text { Yak1501-2085 }\end{array}$ & $\begin{array}{l}\text { San1-1500+Yak1501- } \\
\text { 2085-pBPSUw }\end{array}$ & Yak18C05_1021-2085-For & PBPGUw-GIBSON-Rev \\
\hline 14. & Yak_1-500 & $\begin{array}{l}\text { 18C05_yak_full- } \\
\text { pBPSUw }\end{array}$ & PBPGUw-GIBSON-For & Yak18C05_1-550-Rev \\
\hline 15. & San_501-1500 & $\begin{array}{l}\text { 18C05_san_full- } \\
\text { pBPSUw }\end{array}$ & $\begin{array}{l}\text { San18C05_500-2079bp- } \\
\text { For }\end{array}$ & San18C05_1-1609-Rev \\
\hline 16. & San_501-1000 & $\begin{array}{l}\text { 18C05_san_full- } \\
\text { pBPSUW }\end{array}$ & San18C05_500-2079-For & San18C05_1-1070-Rev \\
\hline 17. & SNP-970/1 & $\begin{array}{l}\text { 18C05_yak_full- } \\
\text { pBPSUw }\end{array}$ & PBPGUw-GIBSON-For & $\begin{array}{l}\text { Yak_1078-2086_San- } \\
\text { SNP970-Rev }\end{array}$ \\
\hline 18. & SNP-970/2 & $\begin{array}{l}\text { 18C05_yak_full- } \\
\text { pBPSUw }\end{array}$ & $\begin{array}{l}\text { Yak_1-1078_San-SNP970- } \\
\text { For }\end{array}$ & PBPGUw-GIBSON-Rev \\
\hline 19. & SNP-1429/1 & $\begin{array}{l}\text { 18C05_yak_full- } \\
\text { pBPSUw }\end{array}$ & PBPGUw-GIBSON-For & $\begin{array}{l}\text { Yak_1535-2086_San- } \\
\text { SNP1429-Rev }\end{array}$ \\
\hline 20. & SNP-1429/2 & 18C05_yak_full- & Yak_1-1535_San- & PBPGUw-GIBSON-Rev \\
\hline
\end{tabular}




\begin{tabular}{|l|lll|l|}
\hline & & pBPSUw & SNP1429-For & \\
\hline 21. & SNP-1507/1 & $\begin{array}{l}\text { 18C05_yak_full- } \\
\text { pBPSUw }\end{array}$ & PBPGUw-GIBSON-For & $\begin{array}{l}\text { Yak_1613-2086-San- } \\
\text { SNP1507-Rev }\end{array}$ \\
\hline 22. & SNP-1507/2 & $\begin{array}{l}\text { 18C05_yak_full- } \\
\text { pBPSUw }\end{array}$ & $\begin{array}{l}\text { Yak_1-1613-San- } \\
\text { SNP1507-For }\end{array}$ & $\begin{array}{l}\text { PBPGUw-GIBSON-Rev } \\
\text { 23. }\end{array}$ \\
\hline SNP-1775/1 & $\begin{array}{l}\text { 18C05_yak_full- } \\
\text { pBPSUw }\end{array}$ & PBPGUw-GIBSON-For & $\begin{array}{l}\text { Yak_1880-2086-San- } \\
\text { SNP1775-Rev }\end{array}$ \\
\hline 24. & SNP-1775/2 & $\begin{array}{l}\text { 18C05_yak_full- } \\
\text { pBPSUw }\end{array}$ & $\begin{array}{l}\text { Yak_1-1880-San- } \\
\text { SNP1775-For }\end{array}$ & PBPGUw-GIBSON-Rev \\
\hline
\end{tabular}

Table S10. Scheme of Gibson assembly for chimeric constructs

See Table S9. for description of the PCR fragments.

\begin{tabular}{|l|l|}
\hline Construct Name & Assembled PCR Fragments \\
\hline 18C05_SSSY & $1+2$ \\
\hline 18C05_YYSS & $3+4$ \\
\hline 18C05_SSYS & $5+6$ \\
\hline 18C05_SYYY & $7+8$ \\
\hline 18C05_YYYS & $9+10$ \\
\hline 18C05_SSYY & $11+12$ \\
\hline 18C05_YYSY & $3+13$ \\
\hline 18C05_YSYY & $14+16+12$ \\
\hline 18C05_YSSY & $14+15+2$ \\
\hline 18C05yakT970A & $17+18$ \\
\hline 18C05YyakT1429G & $19+20$ \\
\hline 18C05yakA1507G & $21+22$ \\
\hline 18C05yakt1775G & $23+24$ \\
\hline
\end{tabular}

Table S11. List of 18C05yakuba- and 18C05ancestral-SNP-pBPSUw constructs synthesized by GenScript

\begin{tabular}{|l|l|}
\hline Name & Construction \\
\hline 18C05_Anc-pBPSUw & Ordered at GenScript \\
\hline 18C05_AncT1008C-pBPSUw & Ordered at GenScript, nucleotide T at position 1008 replaced to C \\
\hline 18C05_AncT1429G-pBPSUw & Ordered at GenScript, nucleotide T at position 1429 replaced to G \\
\hline 18C05_AncT1482C-pBPSUw & Ordered at GenScript, nucleotide T at position 1482 replaced to C \\
\hline 18C05_AncA1507G-pBPSUw & Ordered at GenScript, nucleotide A at position 1507 replaced to C \\
\hline 18C05_AncT1775G-pBPSUw & Ordered at GenScript, nucleotide T at position 1775 replaced to C \\
\hline 18C05_yakG869A-pBPSUw & Ordered at GenScript, nucleotide G at position 869 replaced to A \\
\hline
\end{tabular}




\begin{tabular}{|l|l|}
\hline 18C05_yakT1008C-pBPSUw & $\begin{array}{l}\text { pUC57 was ordered at GenScript and subcloned into pBPSUw using } \\
\text { Gibson Assembly, nucleotide T at position } 1008 \text { replaced to C }\end{array}$ \\
\hline 18C05_yakT1482C-pBPSUw & $\begin{array}{l}\text { pUC57 was ordered at GenScript and subcloned into pBPSUw using } \\
\text { Gibson Assembly, nucleotide T at position } 1482 \text { replaced to C }\end{array}$ \\
\hline 18C05_AncG869A-pBPSUw & $\begin{array}{l}\text { pUC57 was ordered at GenScript and subcloned into pBPSUw using } \\
\text { Gibson Assembly, nucleotide G at position } 869 \text { replaced to A }\end{array}$ \\
\hline 18C05_AncT670G-pBPSUw & $\begin{array}{l}\text { pUC57 was ordered at GenScript and subcloned into pBPSUw using } \\
\text { Gibson Assembly, nucleotide T at position } 670 \text { replaced to G }\end{array}$ \\
\hline 18C05_Anc-SNPall-pBPSUw & $\begin{array}{l}\text { pUC57 was ordered at GenScript and subcloned into pBPSUw using } \\
\text { Gibson Assembly, nucleotide substitutions at the indicated positions: } \\
\text { T670G, G869A, T1008C, T1429G, T1482C, A1507G, T1775G }\end{array}$ \\
\hline
\end{tabular}




\section{Table S12. JASPAR binding scores for 6 loci of interest within the $18 C 05$ region}

Predicted transcription factors (TF) binding sites and their binding scores are shown for 3 loci of interest within the $18 C 05$ region which underwent $D$. santomea-specific substitutions associated with a decrease in hypandrial bristle number For each position the left column indicates binding scores for the D. yakuba sequence and the right column for the $D$. santomea sequence. All the TF predicted by JASPAR with binding scores higher than 0.9 for at least one locus are shown. "-" indicates cases where binding scores of the D. yakuba sequence is less than 0.9. In red are binding scores that were changed by the mutation. We found for T1429G a gain of two repressors (B-H1 and B-H2), for A1507G a loss of several TF binding sites (AWH, DDX, LBE, LBL, LIM3 and VVL), for T1775G a gain of ARA and MIRR binding and a loss of BR-Z and ABD-B binding sites.

\begin{tabular}{|c|c|c|c|c|c|c|}
\hline TF & $\begin{array}{l}1429 \\
\text { (yak) }\end{array}$ & $\begin{array}{c}\text { T1429G } \\
\text { (san) }\end{array}$ & $\begin{array}{l}1507 \\
\text { (yak) }\end{array}$ & $\begin{array}{c}\text { A1507G } \\
\text { (san) }\end{array}$ & $\begin{array}{l}1775 \\
\text { (yak) }\end{array}$ & $\begin{array}{c}\text { T1775G } \\
\text { (san) }\end{array}$ \\
\hline Abd_B & - & - & - & - & 0.92 & 0.78 \\
\hline ara & - & - & & - & 0.73 & 0.95 \\
\hline Awh & - & - & 0.94 & 0.85 & - & - \\
\hline B-H1 & 0.64 & 0.93 & - & - & - & - \\
\hline B-H2 & 0.67 & 0.91 & - & - & - & - \\
\hline Br-Z2 & - & - & - & - & 0.9 & 0.62 \\
\hline CG1569G-RA & - & - & 0.94 & 0.94 & - & - \\
\hline Dbx & - & - & 0.94 & 0.87 & & \\
\hline lbe & - & - & 0.95 & 0.74 & & \\
\hline lbl & - & - & 0.93 & 0.74 & & \\
\hline $\operatorname{Lim3}$ & - & - & 0.92 & 0.85 & & \\
\hline mirr & - & - & - & - & 0.71 & 0.93 \\
\hline onecut & - & - & 0.99 & 0.98 & - & - \\
\hline vvl & - & - & 0.92 & 0.83 & - & - \\
\hline
\end{tabular}


Table S13. Hypandrial bristle phenotypes in $A B D-B R N A$ lines and mitotic clones $A b d-B R N A i$ was induced with different $G A L 4$ drivers and $A b d-B^{M 1}$ and $A b d-B^{D 18}$ mutations were used in mitotic clones. The number of dissected flies and recognizable hypandrium with a shape similar to wild-type are shown. The number of hypandrium with $0,1,2,3$ or with 2 misplaced bristles are shown in separate columns. Different bristle phenotypes are indicated with the following labels: $\mathrm{n}$ : normal, $\mathrm{t}$ : thin, s: shorter than wild-type, M: Minute (thinner and slightly shorter than wild-type), y+: non yellow, y-: yellow, R: 2 bristles on the right, L: 2 bristles on the left.

\begin{tabular}{|c|c|c|c|c|c|c|c|}
\hline Genotype & $\begin{array}{c}\text { Number of } \\
\text { dissected } \\
\text { flies }\end{array}$ & $\begin{array}{l}\text { Number of } \\
\text { recognizable } \\
\text { hypandrium }\end{array}$ & 0 bristle & 1 bristle & 2 bristles & 3 bristles & $\underset{\text { bristle }}{2 \text { misplaced }}$ \\
\hline$A b d-B . R N A i^{26746} / N P 6333-G A L 4$ & 50 & 0 & & & & & \\
\hline$A b d-B . R N A i^{26746} / N P 5130-G A L 4$ & 50 & 0 & & & & & \\
\hline Abd-B.RNAi ${ }^{51167} / N P 6333-G A L 4$ & 50 & 9 & & $1(\mathrm{t}, \mathrm{s})$ & $6(t, s)$ & $1(t, s)$ & $1(\mathrm{~L})$ \\
\hline$A b d-B . R N A i^{51167} / N P 5130-G A L 4$ & 50 & 11 & & & $9(\mathrm{t}, \mathrm{s})$ & & $1(\mathrm{~L}), 1(\mathrm{R})$ \\
\hline $\begin{array}{l}\text { Abd-B.RNAi }{ }^{51167} / G M R 18 C 05- \\
\text { GAL4 }\end{array}$ & 10 & 10 & & & $7(\mathrm{n}), 3(\mathrm{t})$ & & \\
\hline 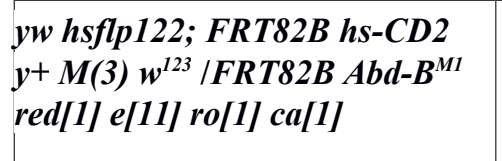 & 30 & 10 & 2 & $2\left({\left.\mathrm{M}, \mathrm{y}^{+}\right)}\right.$ & $\begin{array}{c}2\left(\mathrm{M}, \mathrm{y}^{+}\right) \\
1\left(\mathrm{M}, \mathrm{y}^{-}\right) \\
1\left(\mathrm{M}, \mathrm{y}^{+} ; \mathrm{M}, \mathrm{y}^{-}\right)\end{array}$ & & $2\left(\mathrm{R}, \mathrm{M}, \mathrm{y}^{-}\right)$ \\
\hline $\begin{array}{l}y w \\
y s f l p 122 ; \text { FRT82B } \\
y+M(3) w^{123} / F R T 82 B \text { Abd-B } \\
y^{D 18}\end{array}$ & 52 & 2 & 1 & $1\left(\mathrm{y}^{+}\right)$ & & & \\
\hline
\end{tabular}




\section{AUXILIARY DATA FILES}

Data S1. QTL-data (separate file)

3 Data for Fig. 2.

Data S2. Hypandrial bristle number with $18 C 05-G A L 4$ constructs (separate file)

Data for Fig. 3.

Data S3. Hypandrial bristle number with $18 C 05$-sc constructs (separate file) Data for Fig. 3., Fig S3., Fig S4.

Data S4. Sex comb tooth number with various $18 C 05 y a k$-sc constructs (separate file)

Data S5. Genital bristle numbers in D. yakuba and D. santomea strains (separate file) Data for Fig. S1.

Data S6. Hypandrial bristle number with $18 C 05$ melanogaster-GAL4 constructs (separate file) Data for Fig. S5.

Data S7. Hypandrial bristle number with chimeric constructs (separate file) Data for Fig. S8.

Data S8. Sex comb tooth numbers in D. yakuba, D. santomea and in D. melanogaster Canton S and sc mutants (separate file)

Data for Fig. S9.

Data S9. Fractional occupancy data (separate file)

Data for Fig. S11. EMSA shift intensity values measured by ImageJ and the calculated fractional occupancy.

Data S10. Number of GFP-positive cells in $5 \mathrm{~h}$ APF pupal legs of $D$. melanogaster flies of genotype 18C05yak-GFP or 18C05yakT1775G-GFP or 18C05san-GFP (separate file)

Data for Fig. 4. 


\section{SUPPLEMENTAL REFERENCES}

29. Andolfatto, P., Davison, D., Erezyilmaz, D., Hu, T.T., Mast, J., Sunayama-Morita, T., and Stern, D.L. (2011). Multiplexed shotgun genotyping for rapid and efficient genetic mapping. Genome Res. 21, 610-617.

30. Broman, K.W., and Sen, S. (2009). A Guide to QTL Mapping with R/qtl 1st ed. (Springer).

31. Broman, K.W., Wu, H., Sen, S., and Churchill, G.A. (2003). R/qtl: QTL mapping in experimental crosses. Bioinformatics 19, 889-890.

32. Haley, C.S., and Knott, S.A. (1992). A simple regression method for mapping quantitative trait loci in line crosses using flanking markers. Heredity (Edinb) 69, 315-324.

33. Venken, K.J.T., Popodi, E., Holtzman, S.L., Schulze, K.L., Park, S., Carlson, J.W., Hoskins, R.A., Bellen, H.J., and Kaufman, T.C. (2010). A molecularly defined duplication set for the X chromosome of Drosophila melanogaster. Genetics 186, 1111-1125.

34. Turissini, D.A., McGirr, J.A., Patel, S.S., David, J.R., and Matute, D.R. (2017). The rate of evolution of postmatingprezygotic reproductive isolation in Drosophila. Mol. Biol. Evol.

35. Schneider, C.A., Rasband, W.S., and Eliceiri, K.W. (2012). NIH Image to ImageJ: 25 years of image analysis. Nature methods 9, 671-675.

36. Taylor, B.J. (1989). Sexually dimorphic neurons in the terminalia of Drosophila melanogaster: I. Development of sensory neurons in the genital disc during metamorphosis. J. Neurogenet. 5, 173-192.

37. Dietzl, G., Chen, D., Schnorrer, F., Su, K.-C., Barinova, Y., Fellner, M., Gasser, B., Kinsey, K., Oppel, S., Scheiblauer, S., et al. (2007). A genome-wide transgenic RNAi library for conditional gene inactivation in Drosophila. Nature 448, $151-156$.

38. Jenett, A., Rubin, G.M., Ngo, T.-T., Shepherd, D., Murphy, C., Dionne, H., Pfeiffer, B.D., Cavallaro, A., Hall, D., Jeter, J., et al. (2012). A GAL4-driver line resource for Drosophila neurobiology. Cell reports 2, 991-1001.

39. Gibson, D.G., Young, L., Chuang, R.-Y., Venter, J.C., Hutchison, C.A., and Smith, H.O. (2009). Enzymatic assembly of DNA molecules up to several hundred kilobases. Nature methods 6, 343-345.

40. Bryne, J.C., Valen, E., Tang, M.-H.E., Marstrand, T., Winther, O., da Piedade, I., Krogh, A., Lenhard, B., and Sandelin, A. (2007). JASPAR, the open access database of transcription factor-binding profiles: new content and tools in the 2008 update. Nucleic acids research 36, D102-D106.

41. Zhu, L.J., Christensen, R.G., Kazemian, M., Hull, C.J., Enuameh, M.S., Basciotta, M.D., Brasefield, J.A., Zhu, C., Asriyan, Y., Lapointe, D.S., et al. (2010). FlyFactorSurvey: a database of Drosophila transcription factor binding specificities determined using the bacterial one-hybrid system. Nucleic acids research 39, D111-D117.

42. Crawley, M.J. (2012). The R book (John Wiley \& Sons).

43. Hilbe, J.M. (2014). Modeling Count Data (Cambridge University Press).

44. Zuur, A., Ieno, E.N., Walker, N., Saveliev, A.A., and Smith, G.M. (2011). Mixed Effects Models and Extensions in Ecology with R Softcover reprint of hardcover 1st ed. 2009 edition. (New York, NY: Springer).

45. Team, R.C. (2016). R: A language and environment for statistical (Vienna, Austria) Available at: http://www.Rproject.org/.

46. Bates, D., Mächler, M., Bolker, B., and Walker, S. (2014). Fitting linear mixed-effects models using lme4. arXiv preprint arXiv:1406.5823. 
47. Hothorn, T., Bretz, F., and Westfall, P. (2008). Simultaneous inference in general parametric models. Biometrical journal 50, 346-363.

48. Bretz, F., Hothorn, T., and Westfall, P. (2010). Multiple comparisons using R (CRC Press).

49. Holm, S. (1979). A simple sequentially rejective multiple test procedure. Scandinavian journal of statistics, 65-70.

50. Frangioni, J.V., and Neel, B.G. (1993). Solubilization and purification of enzymatically active glutathione S-transferase (pGEX) fusion proteins. Analytical biochemistry 210, 179-187.

51. Fan, Y.-J., Gittis, A.H., Juge, F., Qiu, C., Xu, Y.-Z., and Rabinow, L. (2014). Multifunctional RNA Processing Protein SRm160 Induces Apoptosis and Regulates Eye and Genital Development in Drosophila. Genetics 197, 1251-1265.

52. Chatterjee, S.S., Uppendahl, L.D., Chowdhury, M.A., Ip, P.-L., and Siegal, M.L. (2011). The female-specific doublesex isoform regulates pleiotropic transcription factors to pattern genital development in Drosophila. Development 138, 1099-1109.

53. Skaer, N., Pistillo, D., and Simpson, P. (2002). Transcriptional heterochrony of scute and changes in bristle pattern between two closely related species of blowfly. Developmental biology 252, 31-45.

54. Casanova, J., Sánchez-Herrero, E., and Morata, G. (1986). Identification and characterization of a parasegment specific regulatory element of the abdominal-B gene of Drosophila. Cell 47, 627-636.

55. Hopmann, R., Duncan, D., and Duncan, I. (1995). Transvection in the iab-5, 6, 7 region of the bithorax complex of Drosophila: homology independent interactions in trans. Genetics 139, 815-833.

56. Xu, T., and Rubin, G.M. (1993). Analysis of genetic mosaics in developing and adult Drosophila tissues. Development $117,1223-1237$.

57. Estrada, B., and Sánchez-Herrero, E. (2001). The Hox gene Abdominal-B antagonizes appendage development in the genital disc of Drosophila. Development 128, 331-339.

58. Maroni, G. and S.C. Stamey (1983). Developmental profile and tissue distribution of alcohol dehydrogenase. Drosophila Information Service 59.

59. Andres, A.J., and Thummel, C.S. (1994). Methods for quantitative analysis of transcription in larvae and prepupae. Methods Cell Biol. 44, 565-573.

59b. Corson, F., Couturier, L., Rouault, H., Mazouni, K., \& Schweisguth, F. (2017). Self-organized Notch dynamics generate stereotyped sensory organ patterns in Drosophila. Science, 356(6337), eaai7407.

60. Campuzano, S., Carramolino, L., Cabrera, C.V., Ruíz-Gómez, M., Villares, R., Boronat, A., and Modolell, J. (1985). Molecular genetics of the achaete-scute gene complex of D. melanogaster. Cell 40, 327-338.

61. Carramolino, L., Ruiz-Gomez, M., del Carmen Guerrero, M., Campuzano, S., and Modolell, J. (1982). DNA map of mutations at the scute locus of Drosophila melanogaster. The EMBO journal 1, 1185.

62. Cubas, P., De Celis, J.F., Campuzano, S., and Modolell, J. (1991). Proneural clusters of achaete-scute expression and the generation of sensory organs in the Drosophila imaginal wing disc. Genes \& Development 5, 996-1008.

63. Kvon, E.Z., Kazmar, T., Stampfel, G., Yáñez-Cuna, J.O., Pagani, M., Schernhuber, K., Dickson, B.J., and Stark, A. (2014). Genome-scale functional characterization of Drosophila developmental enhancers in vivo. Nature 512, 91-95., 91-95. 